DOI 10.4171/JEMS/407

Jaeyoung Byeon · Kazunaga Tanaka

\title{
Semi-classical standing waves for nonlinear Schrödinger equations at structurally stable critical points of the potential
}

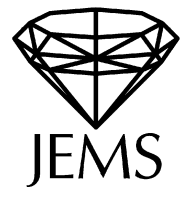

Received September 6, 2011

Abstract. We consider a singularly perturbed elliptic equation

$$
\varepsilon^{2} \Delta u-V(x) u+f(u)=0, \quad u(x)>0 \text { on } \mathbb{R}^{N}, \quad \lim _{|x| \rightarrow \infty} u(x)=0,
$$

where $V(x)>0$ for any $x \in \mathbb{R}^{N}$. The singularly perturbed problem has corresponding limiting problems

$$
\Delta U-c U+f(U)=0, \quad U(x)>0 \text { on } \mathbb{R}^{N}, \quad \lim _{|x| \rightarrow \infty} U(x)=0, \quad c>0 .
$$

Berestycki-Lions [3] found almost necessary and sufficient conditions on the nonlinearity $f$ for existence of a solution of the limiting problem. There have been endeavors to construct solutions of the singularly perturbed problem concentrating around structurally stable critical points of the potential $V$ under possibly general conditions on $f$. In this paper, we prove that under the optimal conditions of Berestycki-Lions on $f \in C^{1}$, there exists a solution concentrating around topologically stable positive critical points of $V$, whose critical values are characterized by minimax methods.

\section{Introduction}

During the last several decades there have been a great deal of work on the semi-classical standing waves of nonlinear Schrödinger equations. A standing wave of a nonlinear Schrödinger equation

$$
i \hbar \frac{\partial \psi}{\partial t}+\frac{\hbar^{2}}{2} \Delta \psi-V(x) \psi+f(\psi)=0, \quad(t, x) \in \mathbb{R} \times \mathbb{R}^{N}
$$

is a solution of the form $\psi(x, t)=\exp (-i E t / \hbar) v(x)$ for some $E \in \mathbb{R}$ and a real valued function $v$. Here $\hbar$ denotes the Planck constant and $i$ the imaginary unit. We assume that

J. Byeon: Department of Mathematical Sciences, KAIST, 291 Daehak-ro, Yuseong-gu, Daejeon 305-701, Republic of Korea; e-mail: byeon@kaist.ac.kr

K. Tanaka: Department of Mathematics, School of Science and Engineering, Waseda University, 3-4-1 Ohkubo, Shijuku-ku, Tokyo 169-8555, Japan; e-mail: kazunaga@waseda.jp 
$f(\exp (i \theta) s)=\exp (i \theta) f(s)$ for $s, \theta \in \mathbb{R}$. Then $\psi(x, t)=\exp (-i E t / \hbar) v(x)$ is a solution of (1) if and only if the function $v$ satisfies

$$
\frac{\hbar^{2}}{2} \Delta v-(V(x)-E) v+f(v)=0 \quad \text { in } \mathbb{R}^{N} .
$$

We are interested in the semi-classical state, that is, the case where $\hbar>0$ is sufficiently small. Thus, replacing $V-E$ by $V$ for convenience, we study the equation

$$
\varepsilon^{2} \Delta v-V(x) v+f(v)=0 \quad \text { in } \mathbb{R}^{N},
$$

where $\varepsilon>0$ is sufficiently small. Throughout the paper, we assume that $N \geq 2$ and the potential $V$ satisfies

(V1) $V \in C\left(\mathbb{R}^{N}, \mathbb{R}\right)$ and $\underline{V} \equiv \inf _{\mathbb{R}^{N}} V(x)>0$.

By a change of variables $x \mapsto \varepsilon x$, we see that the singularly perturbed problem (2) is equivalent to

$$
\Delta u-V(\varepsilon x) u+f(u)=0 \quad \text { in } \mathbb{R}^{N} .
$$

We note that for each $x_{0} \in \mathbb{R}^{N}$ and $R>0, V_{\varepsilon}(x) \equiv V(\varepsilon x)$ converges uniformly to $V\left(x_{0}\right)$ on $B\left(x_{0} / \varepsilon, R\right)$ as $\varepsilon \rightarrow 0$. Thus for each $x_{0} \in \mathbb{R}^{N}$, we have a formal limiting problem

$$
\Delta U(x)-V\left(x_{0}\right) U(x)+f(U(x))=0, \quad x \in \mathbb{R}^{N} .
$$

Berestycki-Lions proved in their classical paper [3] (see also [4] for case $N=2$ ) that there exists a positive least energy solution $U \in H^{1}\left(\mathbb{R}^{N}\right)$ of (4) if the function $f \in$ $C(\mathbb{R}, \mathbb{R})$ satisfies the following conditions with $V\left(x_{0}\right)=m$ :

(f1) $f(0)=\lim _{t \rightarrow 0} f(t) / t=0$;

(f2) if $N \geq 3$, then there exist $C>0$ and $p \in(1,(N+2) /(N-2))$ such that $|f(t)| \leq$ $C\left(1+t^{p}\right)$ for all $t \in \mathbb{R}^{+}$; if $N=2$, for any $\alpha>0$, there exists $C_{\alpha}>0$ such that $|f(t)| \leq C_{\alpha} \exp \left(\alpha t^{2}\right)$ for all $t \geq 0$;

(f3) there exists $t_{0}>0$ such that $\frac{1}{2} m t_{0}^{2}<F\left(t_{0}\right)$, where $F(t)=\int_{0}^{t} f(s) d s$.

For any solution $U \in H^{1}\left(\mathbb{R}^{N}\right)$ of (4), we have the following Pohozaev's identity:

$$
\frac{N-2}{2} \int_{\mathbb{R}^{N}}|\nabla U|^{2} d x+N \int_{\mathbb{R}^{N}}\left[V\left(x_{0}\right) \frac{U^{2}}{2}-F(U)\right] d x=0 .
$$

From this identity, we see that (f3) is a necessary condition for existence of a solution $U$ of (4). Condition (f2) is also necessary in the sense that if $N \geq 3$ and $f(t)=t^{p}$ with $p \geq(N+2) /(N-2)$, then there exist no solutions of (4) in $H^{1}\left(\mathbb{R}^{N}\right)$.

On the other hand, we note that for any positive solution $U_{x_{0}} \in H^{1}\left(\mathbb{R}^{N}\right)$ of (4), $\left\{U_{x_{0}}\left(\cdot-x_{0} / \varepsilon\right) \mid x_{0} \in \mathbb{R}^{N}\right\}$ is a set of approximate solutions of (3) for small $\varepsilon>0$. In a pioneering work [20], Floer and Weinstein showed that when $N=1, V \in C^{2}\left(\mathbb{R}^{N}\right)$, $V^{\prime}\left(x_{0}\right)=0, V^{\prime \prime}\left(x_{0}\right) \neq 0$ and $f(u)=u^{3}$, there exists a positive solution $u_{\varepsilon} \in H^{1}(\mathbb{R})$ of (3) such that for a maximum point $x_{\varepsilon} \in \mathbb{R}$ of $u_{\varepsilon}, \lim _{\varepsilon \rightarrow 0} \varepsilon x_{\varepsilon}=x_{0}$ and $u_{\varepsilon}\left(\cdot+x_{\varepsilon}\right)$ converges uniformly to $U_{x_{0}}$ as $\varepsilon \rightarrow 0$. In [20], Floer-Weinstein adopted a Lyapunov-Schmidt 
reduction method which requires a linearized nondegeneracy of a solution for a limiting problem. The linearized nondegeneracy means that if $\Delta \phi-V\left(x_{0}\right) \phi+f^{\prime}\left(U_{x_{0}}\right) \phi=0$, then $\phi$ must be of the form $\phi=\sum_{i=1}^{N} a_{i} \partial U_{x_{0}} / \partial x_{i}$ for some $a_{i} \in \mathbb{R}$. Motivated by the approach in [20], many authors have obtained further refined results in higher dimensions for more general $f$ and more general types of critical points of $V$ (see $[1,2,19,27,28,32$, 33 ] and references therein). When we use the Lyapunov-Schmidt finite-dimensional reduction method for (3), we need the linearized nondegeneracy condition, which is known to hold only for a restricted class of $f$ if $N \geq 2$; a recent result [11] says that we need at least the monotonicity of $\left(V\left(x_{0}\right) t-f^{\prime}(t) t\right) /\left(V\left(x_{0}\right) t-f(t)\right)$ for $t>t_{0}$, where $t_{0}>0$ is the first positive zero of $V\left(x_{0}\right) t-f(t)=0$, which is much stronger than (f3). Even though there is such a restriction on the nonlinearity when we apply the reduction method, the Lyapunov-Schmidt reduction method is a very powerful tool when we construct very subtle (highly unstable) solutions with continuum peaks as we can see in [19]. When the linearized nondegeneracy condition does not hold, a refined finite-dimensional reduction method was developed by Dancer in [13] to get solutions corresponding to an isolated (topologically) nondegenerate critical point of $V$ for a singularly perturbed Dirichlet problem on a bounded domain. But the refined finite-dimensional reduction method still requires some type of nondegeneracy for the limiting problem.

In the other direction, a variational approach which does not require the nondegeneracy condition for the limiting problem (4) was initiated by Rabinowitz [35] and developed further by several authors (see $[10,14,15,17,23,26])$. However the previous works still require stronger conditions for $f$ than (f1)-(f3). In recent papers [8], [9], we managed to prove the existence of a solution of (2) concentrating around local minimum points of $V$ under the optimal conditions (f1)-(f3). It has been a challenging problem to construct a solution of (2) concentrating around general critical points under the Berestycki-Lions conditions (f1)-(f3).

In this paper, we resolve the problem for $f \in C^{1}$ satisfying (f1)-(f3) by developing a new variational approach. Our variational approach in this paper is a further development of the approach in [8], quite different from the approaches of del Pino and Felmer in [15, 17], and we use a localized deformation argument in a neighborhood of a set of approximate solutions. In the variational approach of [8], the characteristic of local minimum points of $V$ makes it rather easy to get a lower estimate of a local mountain pass level and a positive lower bound, independent of $\varepsilon>0$, of the gradient norm of an energy functional in the intersection of an annular neighborhood of approximate solutions and a level set where the energy level is less than or equal to the maximum of a good initial path. Then, if there are no solutions in a neighborhood of approximate solutions, we get a path through the gradient flow where the maximum energy is strictly less than the lower estimate of the local mountain pass level; this contradicts the lower energy estimate and proves the existence of a solution.

On the other hand, for general critical points of $V$, the situation is much more complicated and we need totally new ideas. When we try to find a solution, a critical point of the corresponding functional, through the variational approach, deformation arguments play important roles. A deformation argument using the gradient flow of the energy functional is the most common and powerful tool in variational methods. If we use only the 
deformation argument which comes from the gradient of an energy functional in our problem, we need some lower estimate for the gradient norm $\left\|\Gamma_{\varepsilon}^{\prime}(u)\right\|$ when the center of mass is away from the critical points of $V$. Such a lower estimate was obtained by del Pino and Felmer [17] for the gradient flow (starting from good points) on the $\mathrm{Ne}$ hari manifold, where they require quite strong conditions on the nonlinearity $f$ and the potential $V$.

In this paper, to bypass the obstacle in obtaining a lower gradient estimate for a general type of nonlinearity $f$ and general potential $V$, we devise a different kind of gradient flow on a Sobolev space which comes from a (pseudo) gradient flow of the potential $V$. Thus we use two kinds of gradient flow in this paper. Then, inspired by the Trotter product formula, we iterate the composition of the two gradient flows for our deformation argument. More precisely, since the gradient flow of the energy functional does not preserve concentration of functions due to its diffusing effect, we need one more continuous operation on a small neighborhood of a set of approximate solutions which makes the tails of the functions small. Then, if we compose the gradient flow of the energy functional, the operation keeping tails small and the gradient flow of the potential $V$, the composite operator $I$ keeps the concentration property of functions. This concentration property is one of the essential ingredients of our deformation argument in this paper.

When $N=1$, there is a necessary and sufficient condition on $f$ for existence of a solution of (4) in $H^{1}(\mathbb{R})$. Our argument in this paper is also valid in the case $N=1$ under the necessary and sufficient condition on $f$ and $V \in C^{0,1}$. But, there exists a unique solution, up to translation, and it is nondegenerate if $f \in C^{1}(\mathbb{R})$. This implies that the Lyapunov-Schmidt reduction method is also valid when $f \in C^{1}(\mathbb{R})$ and $V \in C^{1}\left(\mathbb{R}^{N}\right)$. (For a different approach to find more general types of solutions, refer to [18].) Thus, in this paper, we just consider the case $N \geq 2$. Moreover, since it is proved in [8] that there exists a solution concentrating around local minimum points of $V$, from now on, we consider non-minimum critical points of $V$. Our typical result is the following.

Theorem 1. Suppose that $f \in C^{1}$ satisfies conditions (f1)-(f3), and that the potential $V \in C^{1}\left(\mathbb{R}^{N}\right)$ satisfies $(\mathrm{V} 1)$. Let $\mathcal{M}$ be an isolated saddle point or an isolated set of local maximum points of $V$. Then for sufficiently small $\varepsilon>0$, there exists a positive solution $v_{\varepsilon}$ of (2) such that for a maximum point $x_{\varepsilon}$ of $v_{\varepsilon}$, there exist constants $C, c>0$, independent of small $\varepsilon>0$, satisfying $v_{\varepsilon}(x) \leq C \exp \left(-\frac{c}{\varepsilon}\left|x-x_{\varepsilon}\right|\right)$ and

$$
\lim _{\varepsilon \rightarrow 0} \operatorname{dist}\left(x_{\varepsilon}, \mathcal{M}\right)=0 \text {. }
$$

Moreover, $w_{\varepsilon}(x) \equiv v_{\varepsilon}\left(\varepsilon x+x_{\varepsilon}\right)$ converges along a subsequence uniformly to a radially symmetric least energy solution of $\Delta w-m w+f(w)=0, w>0$ in $\mathbb{R}^{N}$ with $m=V\left(x_{0}\right)$ for some $x_{0} \in \mathcal{M}$.

In the next section, we give a more general existence result for more general types of critical points together with some typical examples.

This paper is organized as follows.

First in Section 2, we give a refined existence result with some examples. To prove the refined result, we define a set of approximate solutions in Section 3. Then, in Section 4, 
we define the center of mass for elements in a neighborhood of the set of approximate solutions. In Section 5, we define a new neighborhood of the set of approximate solutions using the center of mass. Then, we construct a good starting surface in Section 6. In Section 7, we get a lower estimate of the gradient norm of an energy functional for elements which are not too close to the approximate solutions and whose center of mass stays away from the critical points of $V$. In Section 8, we consider a map on the new neighborhood which does not increase the energy and projects to a class of functions with exponential decay away from the center of mass. Then, in Section 9, we construct a translation map on the new neighborhood which also does not increase the energy. Lastly, in Section 10, if there is no solution in the new neighborhood, iterating the initial surface by the gradient flow of the associated energy functional, the map keeping tails small and the translation map, we get a surface on which the maximal energy is strictly less than the least energy level. Then, by an intersection lemma, we get a contradiction.

\section{Statement of a refined result and some examples}

For any set $A \subset \mathbb{R}^{N}$ and $d>0$, we define $A^{d} \equiv\left\{x \in \mathbb{R}^{N} \mid \operatorname{dist}(x, A) \leq d\right\}$. We also use the notation

$$
V_{c_{1}}^{c_{2}}=\left\{x \in \mathbb{R}^{N} \mid c_{1} \leq V(x) \leq c_{2}\right\} \quad \text { for } c_{1}<c_{2} .
$$

Now, we consider general types of critical values of $V$ by a minmax argument.

(V2) For $k \geq 1$, there exists a connected bounded open set $O \subset \mathbb{R}^{N}$ with a smooth boundary and a compact $(k-1)$-dimensional manifold $L_{0} \subset O$ without boundary such that, if we define $\mathcal{L}\left(L_{0}\right)$ to be the set of compact connected (orientable) $k$ dimensional manifolds $H$ with boundary $\partial H$ homeomorphic to $L_{0}$, and

$$
\Lambda_{H} \equiv\left\{\varphi \in C(H, O)|\varphi|_{\partial H}: \partial H \rightarrow L_{0} \text { is a homeomorphism }\right\},
$$

then $\mathcal{L}\left(L_{0}\right)$ is not empty and the following strict inequality holds:

$$
m_{0} \equiv \max _{x \in L_{0}} V(x)<m \equiv \inf _{H \in \mathcal{L}\left(L_{0}\right), \varphi \in \Lambda_{H}} \max _{x \in H} V(\varphi(x)) .
$$

A rather different feature in (V2), compared with a standard minmax argument, is that we do not restrict the domain manifolds $H$ to be contained in $\mathbb{R}^{N}$. In case $x_{0}$ is a nondegenerate critical point of $V$, the critical value $m=V\left(x_{0}\right)$ is characterized by the minimax argument in (V2) (see Example 1 below).

The following condition yields the existence of a pseudo-gradient flow of $V$.

(V3) There exists a nonempty compact set $\mathcal{M} \subset\{x \in O \mid V(x)=m\}$ such that for any $d>0$ with $\mathcal{M}^{d} \subset O$, there exist constants $\alpha, \beta, \mu, c_{1}, c_{2}>0$, an open set $\Omega$ satisfying $\mathcal{M} \subset \Omega \subset \Omega^{10 \beta} \subset \mathcal{M}^{d}$ and a map $\Phi \in C([0,1] \times O, O)$ such that

(i) for each $x \in O, V(\Phi(\cdot, x))$ is nonincreasing on $[0,1]$;

(ii) $\Phi(t, x)=x$ if $t=0$ or $x \in L_{0}$;

(iii) $\left|\Phi\left(l^{\prime}, x\right)-\Phi(l, x)\right| \leq \mu\left|l^{\prime}-l\right|$ for $l, l^{\prime} \in[0,1], x \in O$; 
(iv)

$$
\limsup _{h \rightarrow 0+} \frac{V(\Phi(l+h, x)+y)-V(\Phi(l, x)+y)}{h} \leq-\alpha
$$

uniformly for $|y| \leq c_{2}, l \in[0,1)$ and $x \in\left(\Omega^{10 \beta} \cup\left(V_{m-c_{1}}^{m+c_{1}} \cap O\right)\right) \backslash \Omega$.

If $V \in C^{1}\left(\mathbb{R}^{N}\right)$ and $\mathcal{M}$ is an isolated set of critical points, or $V \in C^{N}\left(\mathbb{R}^{N}\right)$, we can construct the required flow $\Phi$ (see Remark 1 below). Here we note that in (7) of (V3), we need (one-side) Lipschitz continuity of $V$ along the flow $\Phi$ in $\left(\Omega^{10 \beta} \cup V_{m-c_{1}}^{m+c_{1}}\right) \backslash \Omega$. Our main result is

Theorem 2. Suppose that $f \in C^{1}$ satisfies conditions (f1)-(f3). Assume that (V1)-(V3) hold. Then for sufficiently small $\varepsilon>0$, there exists a positive solution $v_{\varepsilon}$ of (2) such that for a maximum point $x_{\varepsilon}$ of $v_{\varepsilon}$, there exist constants $C, c>0$, independent of small $\varepsilon>0$, satisfying $v_{\varepsilon}(x) \leq C \exp \left(-\frac{c}{\varepsilon}\left|x-x_{\varepsilon}\right|\right)$ and

$$
\lim _{\varepsilon \rightarrow 0} \operatorname{dist}\left(x_{\varepsilon}, \mathcal{M}\right)=0 .
$$

Moreover, $w_{\varepsilon}(x) \equiv v_{\varepsilon}\left(\varepsilon x+x_{\varepsilon}\right)$ converges along a subsequence uniformly to a radially symmetric least energy solution of $\Delta w-m w+f(w)=0, w>0$ in $\mathbb{R}^{N}$.

Example 1 (standard types of critical points). Condition (V2) holds for standard critical points, that is, nondegenerate critical points, (possibly degenerate) critical points of local mountain pass type and local maxima. For such critical points, there exists a $k$ dimensional manifold $L \subset O$ with $L_{0} \equiv \partial L \subset O$ such that the critical value $m$ can be characterized as

$$
m=\inf _{\varphi \in \Lambda_{L}} \max _{x \in L} V(\varphi(x)),
$$

where $\Lambda_{L}=\left\{\varphi \in C(L, O) \mid \varphi(x)=x\right.$ for $\left.x \in L_{0}\right\}$. We claim that for a critical point $z_{0}$, there exists a continuous map $\pi: O \rightarrow L$ such that

$$
\pi(x)=x \quad \text { for } x \in L
$$

and

$$
V(x) \geq m \quad \text { if } \pi(x)=z_{0},
$$

that is, $\pi^{-1}\left(z_{0}\right) \subset\{x \in O \mid V(x) \geq m\}$. Existence of such a map $\pi$ implies condition (V2). In fact, for any $H \in \mathcal{L}\left(L_{0}\right)$ and $\varphi \in \Lambda_{H}$, we consider the mod 2 degree of the map $\pi \circ \varphi, \operatorname{deg}_{2}(\pi \circ \varphi, H, z)$, which is well defined even for a nonorientable manifold (see [24]). Then it is standard to show that $\operatorname{deg}_{2}(\pi \circ \varphi, H, z)=1$ since $\left.\varphi\right|_{\partial H}: \partial H \rightarrow L_{0}$ is a homeomorphism. This implies that $\max _{x \in H} V(\varphi(x)) \geq m$. Since $H, \varphi$ are arbitrary, recalling (8), we have $m=\inf _{H \in \mathcal{L}\left(L_{0}\right), \varphi \in \Lambda_{H}} \max _{x \in H} V(\varphi(x))$. For the existence of $\pi$, we argue separately.

(i) Suppose $x=0$ is a nondegenerate critical point of $V \in C^{2}\left(\mathbb{R}^{N}\right)$. Then there is a new coordinate system $\left\{y_{1}, \ldots, y_{N}\right\}$ in a small ball $B(0, r)$ such that for some $k \in$ $\{0, \ldots, N\}, V\left(y_{1}, \ldots, y_{N}\right)=V(0)-y_{1}^{2}-\cdots-y_{k}^{2}+y_{k+1}^{2}+\cdots+y_{N}^{2}$. Then we take

$$
L \equiv\left\{\left(y_{1}, \ldots, y_{k}, 0, \ldots, 0\right) \in \mathbb{R}^{N} \mid y_{1}^{2}+\cdots+y_{k}^{2} \leq(r / 2)^{2}\right\},
$$


$L_{0}=\partial L$ and $O=B(0, r)$. Let $\pi: O \rightarrow L$ be the projection map defined by

$$
\pi\left(x_{1}, \ldots, x_{N}\right)= \begin{cases}\left(x_{1}, \ldots, x_{k}, 0, \ldots, 0\right) & \text { for } x_{1}^{2}+\cdots+x_{k}^{2} \leq(r / 2)^{2} \\ \frac{r\left(x_{1}, \ldots, x_{k}, 0, \ldots, 0\right)}{2\left|\left(x_{1}, \ldots, x_{k}, 0, \ldots, 0\right)\right|} & \text { for } x_{1}^{2}+\cdots+x_{k}^{2}>(r / 2)^{2} .\end{cases}
$$

Then $\pi^{-1}(0) \subset\{x \in O \mid V(x) \geq V(0)\}$. Thus, (V2) is satisfied for any nondegenerate critical point of $V$.

(ii) Suppose that $V(x)$ has a local maximum point. Let $\mathcal{M}$ be a compact set which consists of local maximum points and suppose that there exists a connected neighborhood $O$ of $\mathcal{M}$ such that $\partial O$ is smooth and $V(x)=V(y)>V(z)$ for any $x, y \in \mathcal{M}$ and $z \in O \backslash \mathcal{M}$. Then, defining $O^{\delta} \equiv\{x \in O \mid \operatorname{dist}(x, \partial O) \geq \delta\}$, we see that for small $\delta>0$, $\partial\left(O^{\delta}\right)$ is a retract of $O \backslash O^{\delta}$, that is, there is a continuous map $\tilde{\pi}: O \backslash O^{\delta} \rightarrow \partial\left(O^{\delta}\right)$ such that $\tilde{\pi}(x)=x$ for any $x \in \partial\left(O^{\delta}\right)$. We define $L=O^{\delta}$. Then a projection map $\pi: O \rightarrow L$ is defined by $\pi(x)=x$ for $x \in O^{\delta}$ and $\pi(x)=\tilde{\pi}(x)$ for $x \in O \backslash O^{\delta}$. We take a point $z \in \mathcal{M}$. Then, obviously, $\pi^{-1}(z) \subset\{x \in O \mid V(x) \geq V(z)\}$; thus, (V2) is satisfied for any local maximum points of $V$.

(iii) Suppose that $V(x)$ has a critical point of local mountain pass type. Let $m$ be the local mountain pass critical value and suppose that there exist an open set $O$ and $e_{0}, e_{1} \in O$ such that $V\left(e_{0}\right), V\left(e_{1}\right)<m=\inf _{\gamma \in \Gamma} \max _{t \in[-1,1]} V(\gamma(t))$, where $\Gamma=\{\gamma \in$ $\left.C([-1,1], O) \mid \gamma(-1)=e_{0}, \gamma(1)=e_{1}\right\}$. Since $V(x)$ has mountain pass geometry, $V^{-1}((-\infty, m)) \cap O$ has at least two connected components; denote by $W_{0}$ the one to which $e_{0}$ belongs. We note $e_{1} \notin \overline{W_{0}}$. We define a signed distance function from $\partial W_{0}$ by

$$
d(x)= \begin{cases}-\frac{\operatorname{dist}\left(x, \partial W_{0}\right)}{\operatorname{dist}\left(e_{0}, \partial W_{0}\right)} & \text { if } x \in W_{0}, \\ \frac{\operatorname{dist}\left(x, \partial W_{0}\right)}{\operatorname{dist}\left(e_{1}, \partial W_{0}\right)} & \text { if } x \notin W_{0} .\end{cases}
$$

We fix $\gamma_{0} \in \Gamma$ such that $V\left(\gamma_{0}(0)\right)=m$ and set $\pi(x)=\gamma_{0}(\psi(d(x)))$, where $\psi \in C(\mathbb{R})$ is such that $\psi(s)=1$ for $s \in[1, \infty), \psi(s)=s$ for $s \in[-1,1], \psi(s)=-1$ for $s \in(-\infty,-1)$. Then $\pi(x)$ satisfies $\pi\left(e_{0}\right)=e_{0}, \pi\left(e_{1}\right)=e_{1}$ and for $z_{0}=\gamma_{0}(0)$ we have $\pi^{-1}\left(z_{0}\right)=\partial W_{0} \subset\{x \mid V(x)=m\}$.

Example 2 (standard types of linking). Condition (V2) holds in the setting of the classical linking theorem in $\mathbb{R}^{N}$ (see [36, Section II, 8] and [34]). For example, for $0<\rho<$ $\rho_{1}, \rho_{2}$ we set

$$
\begin{aligned}
Q & =\left\{\left(x_{1}, \ldots, x_{k}, 0, \ldots, 0\right) \in \mathbb{R}^{N} \mid x_{1}^{2}+\cdots+x_{k-1}^{2} \leq \rho_{2}^{2}, 0 \leq x_{k} \leq \rho_{1}\right\}, \\
S & =\left\{\left(0, \ldots, 0, x_{k}, \ldots, x_{N}\right) \in \mathbb{R}^{N} \mid x_{k}^{2}+\cdots+x_{N}^{2}=\rho^{2}\right\} .
\end{aligned}
$$

We denote by $L_{0} \equiv \partial Q$ the relative boundary of $Q$ and assume $B\left(0, \rho_{1}+\rho_{2}\right) \subset O$. Then under the assumption

$$
m_{0} \equiv \max _{x \in L_{0}} V(x)<\min _{x \in S} V(x)
$$


(V2) holds. In fact, $Q$ is an element of $\mathcal{L}\left(L_{0}\right)$ and $m$ defined in (6) satisfies

$$
m_{0}<\min _{x \in S} V(x) \leq m
$$

which follows from the fact that

$$
\varphi(H) \cap S \neq \emptyset \quad \text { for all } H \in \mathcal{L}\left(L_{0}\right) \text { and } \varphi \in \Lambda_{H} .
$$

The intersection property can be proved using the degree for the composition of a projection map and $\varphi$. We remark that $m$ defined in (6) satisfies

$$
m \leq \inf _{\varphi \in \Lambda_{Q}} \max _{x \in Q} V(\varphi(x)),
$$

where $\Lambda_{Q}=\left\{\gamma \in C(Q, O) \mid \gamma(x)=x\right.$ for $\left.x \in L_{0}\right\}$.

Example 3 (nonsmooth potential). For a typical example of $V$ which may not be in $C^{1}$ and satisfies (V1)-(V3), we consider a Lipschitz continuous function $\theta: S^{N-1} \rightarrow(0,1)$ and a continuous function $r:[0, \infty) \rightarrow[0,1]$ satisfying $r(0)=0$ and $r(t)-r(s) \geq t-s$ for $0 \leq s \leq t \leq 1$. Then we define $V(x)=2-r(|x|) \theta(x /|x|)$. In this case, we take $\mathcal{M}=$ $\{0\} \subset \mathbb{R}^{N}, O=B(0,1)$ and $L_{0}=B(0,1 / 2)$. Then, defining $\Phi(t, x)=x+t(1-|x|) x$ for $x \in O=B(0,1)$, we see that conditions (V1)-(V3) are satisfied with $m=2$.

Remark 1 (existence of pseudo-gradient flow $\Phi$ ). For $V \in C^{1}$, we let $\mathcal{M} \equiv\{x \in \bar{O}$ | $\nabla V(x)=0, V(x)=m\}$ and assume that $\mathcal{M}$ is a nonempty compact subset of $O$ and

$$
\nabla V(x) \notin\{\lambda n(x) \mid \lambda \leq 0\} \quad \text { for all } x \in \partial O \text { with } V(x)=m,
$$

where $n(x)$ is the unit outward normal vector at $x \in \partial O$. Then (V3) holds if either $m$ is an isolated critical value of $V(x)$, or $V(x)$ is of class $C^{N}$. In fact, if $m$ is an isolated critical value, for $\Omega=\mathcal{M}^{d / 2}$ with small $d>0$, we have

$$
\inf _{x \in \partial \Omega}|\nabla V(x)|>0 \text {. }
$$

If $V(x)$ is of class $C^{N}$, we see that the set of critical values of $V(x)$ has Lebesgue measure 0 in $\mathbb{R}$ by Sard's theorem. For the fixed $d>0$, we can find a small $\delta>0$ such that

$$
\nabla V(x) \neq 0 \quad \text { for } x \in \partial \mathcal{M}^{d / 2} \text { with } V(x) \in[m-\delta, m+\delta] .
$$

We remark that by Sard's theorem there exist regular values $b_{1} \in(m-\delta, m)$ and $b_{2} \in$ $(m, m+\delta)$ of $V$. We set $\Omega=\mathcal{M}^{d / 2} \cap V_{b_{1}}^{b_{2}}$. Then

$$
\mathcal{M} \subset \Omega \text { and } \nabla V(x) \neq 0 \text { on } \partial \Omega .
$$

We refer to [17] for a related argument.

Thus in both cases there exists a neighborhood $\Omega$ such that $\mathcal{M} \subset \Omega \subset \mathcal{M}^{d}$ and $\nabla V(x) \neq 0$ on $\partial \Omega$. For sufficiently small $\beta>0$, we have

$$
\Omega^{10 \beta} \subset \mathcal{M}^{d} \text { and } \inf _{x \in \Omega^{10 \beta} \backslash \Omega}|\nabla V(x)|>0 .
$$


Since we assume (9), by the definition of $\mathcal{M}$, we can find a small $c_{1}>0$ such that

$$
\inf _{x \in\left(O \cap V_{m-2 c_{1}}^{m+2 c_{1}}\right) \backslash \Omega}|\nabla V(x)|>0 .
$$

Now, since (9) holds, for each $x \in \partial O \cap V_{m-2 c_{1}}^{m+2 c_{1}}$, we can find a vector $W(x)$ satisfying

$$
\nabla V(x) \cdot W(x)>0 \quad \text { and } \quad n(x) \cdot W(x)>0 .
$$

Then, through a partition of unity, we can define a pseudo-gradient vector field $\tilde{W}: \bar{O}$ $\{x \mid \nabla V(x)=0\} \rightarrow \mathbb{R}^{N}$ for $V$ such that $\nabla V(x) \cdot \tilde{W}(x)>0$ if $|\nabla V(x)| \neq 0$, and $n(x) \cdot \tilde{W}(x)>0$ for $x \in \partial O \cap V_{m-2 c_{1}}^{m+2 c_{1}}$. We find a function $\tilde{\varphi} \in C_{0}^{\infty}(\bar{O})$ such that $\tilde{\varphi}(x)=1$ for $x \in\left(\bar{O} \cap V_{m-c_{1}}^{m+c_{1}}\right) \backslash \Omega$ and $\tilde{\varphi}$ vanishes in a small neighborhood of $\mathcal{M}$ and $\bar{O} \backslash V_{m-2 c_{1}}^{m+2 c_{1}}$. Then, we can find a solution $\Phi:[0, \infty) \times \bar{O} \rightarrow \bar{O}$ of

$$
\frac{\partial \Phi(t, x)}{\partial t}=-\tilde{\varphi}(\Phi(t, x)) \tilde{W}(\Phi(t, x)), \quad \Phi(0, x)=x
$$

Obviously, (i)-(iii) in (V3) are satisfied for the solution $\Phi$. The property (7) follows from (10)-(12) and $C^{1}$ smoothness of $V$.

Remark 2 (minimax value for smooth potentials). Suppose that $V \in C^{1}$ and $m$ is a minimax value given in (V2). If $V$ satisfies (9), it is not difficult to see that $m$ is a critical value and the corresponding critical point lies in $O$. Moreover we can show that (V3) implies (9), thus if $V \in C^{1}\left(\mathbb{R}^{N}\right)$ satisfies (V2)-(V3), then

$$
\begin{aligned}
& \emptyset \neq\{x \in O \mid \nabla V(x)=0, V(x)=m\} \subset \mathcal{M}, \\
& \nabla V(x) \notin\{\lambda n(x) \mid \lambda \leq 0\} \quad \text { for all } x \in \partial O \text { with } V(x)=m .
\end{aligned}
$$

\section{Preliminaries}

We will consider the following problem equivalent to (2):

$$
\Delta u-V_{\varepsilon}(x) u+f(u)=0, \quad u>0, \quad \lim _{|x| \rightarrow \infty} u(x)=0,
$$

where $V_{\varepsilon}(x)=V(\varepsilon x)$. From now on, we assume that $f(t)=0$ for $t \leq 0$. Then it is easy to see from the maximum principle that any nontrivial solution of (13) is positive. Let $H_{\varepsilon}$ be the completion of $C_{0}^{\infty}\left(\mathbb{R}^{N}\right)$ with respect to the norm

$$
\|u\|_{\varepsilon}=\left(\int_{\mathbb{R}^{N}}\left[|\nabla u|^{2}+V_{\varepsilon} u^{2}\right] d x\right)^{1 / 2} .
$$

We also denote by $\|\cdot\|_{\varepsilon}^{*}$ the corresponding dual norm on $H_{\varepsilon}^{*}$, that is,

$$
\|f\|_{\varepsilon}^{*}=\sup _{\|\varphi\|_{\varepsilon} \leq 1, \varphi \in H_{\varepsilon}}|\langle f, \varphi\rangle| \quad \text { for } f \in H_{\varepsilon}^{*},
$$


where $\langle f, \varphi\rangle$ is the duality product between $H_{\varepsilon}^{*}$ and $H_{\varepsilon}$. We define a norm $\|\cdot\|$ on $H^{1}\left(\mathbb{R}^{N}\right)$ by

$$
\|u\|=\left(\int_{\mathbb{R}^{N}}\left[|\nabla u|^{2}+m u^{2}\right] d x\right)^{1 / 2} .
$$

We clearly have $H_{\varepsilon} \subset H^{1}\left(\mathbb{R}^{N}\right)$. From now on, for any set $B \subset \mathbb{R}^{N}$ and $\varepsilon>0$, we define $B_{\varepsilon} \equiv\left\{x \in \mathbb{R}^{N} \mid \varepsilon x \in B\right\}$. For $u \in H_{\varepsilon}$, let

$$
\Gamma_{\varepsilon}(u)=\frac{1}{2} \int_{\mathbb{R}^{N}}\left[|\nabla u|^{2}+V_{\varepsilon} u^{2}\right] d x-\int_{\mathbb{R}^{N}} F(u) d x .
$$

It is standard to see that $\Gamma_{\varepsilon} \in C^{1}\left(H_{\varepsilon}\right)$ and a critical point of $\Gamma_{\varepsilon}$ corresponds to a solution of (2). Later, we will modify the nonlinearity $f$ so that the functional $\Gamma_{\varepsilon}$ with the modified nonlinearity is of class $C^{2}$ on $H_{\varepsilon}$, and a critical point near a certain approximating solutions set of the modified energy functional is also one of the original energy functional. For any set $\mathcal{A} \subset H_{\varepsilon}, u \in H_{\varepsilon}$ and $\delta>0$, we define

$$
\operatorname{dist}_{\varepsilon}(u, \mathcal{A}) \equiv \inf _{w \in \mathcal{A}}\|u-w\|_{\varepsilon} \quad \text { and } \quad N_{\delta}(\mathcal{A}) \equiv\left\{u \in H_{\varepsilon} \mid \operatorname{dist}_{\varepsilon}(u, \mathcal{A}) \leq \delta\right\} .
$$

For any $c, d \in \mathbb{R}$, we define

$$
\left(\Gamma_{\varepsilon}\right)^{c} \equiv\left\{u \in H_{\varepsilon} \mid \Gamma_{\varepsilon}(u) \leq c\right\}, \quad\left(\Gamma_{\varepsilon}\right)_{d} \equiv\left\{u \in H_{\varepsilon} \mid \Gamma_{\varepsilon}(u) \geq d\right\} .
$$

For the $\beta>0$ and the open set $O$ in conditions (V2) and (V3), we take a large $D>\max \{1,10 \beta\}$ so that $O \subset B(0, D)$, and define

$$
\omega \equiv \max _{|x| \leq 10 D} V(x) / \min _{|x| \leq 10 D} V(x)>1 .
$$

Note that there exist positive constants $m_{1}, m_{2}$ with $m_{1}<m<m_{2}$ such that (f3) is satisfied for any $s \in\left[m_{1}, m_{2}\right]$ replacing $m$ in (f3). As already mentioned, the following equations for $s>0$ are limiting equations of (13):

$$
\Delta u-s u+f(u)=0, \quad u>0, \quad u \in H^{1}\left(\mathbb{R}^{N}\right) .
$$

We define an energy functional for the limiting problem (16) by

$$
L_{S}(u)=\frac{1}{2} \int_{\mathbb{R}^{N}}\left[|\nabla u|^{2}+s u^{2}\right] d x-\int_{\mathbb{R}^{N}} F(u) d x, \quad u \in H^{1}\left(\mathbb{R}^{N}\right) .
$$

In the classical paper [3], the authors proved that for any $s \in\left[m_{1}, m_{2}\right]$, there exists a least energy solution of (16) if (f1)-(f3) are satisfied. Also they showed that each solution $U$ of (16) satisfies Pohozaev's identity

$$
G_{s}(U) \equiv \frac{N-2}{2} \int_{\mathbb{R}^{N}}|\nabla U|^{2} d x+N \int_{\mathbb{R}^{N}}\left[s \frac{U^{2}}{2}-F(U)\right] d x=0 .
$$

This implies that

$$
L_{S}(U)=\frac{1}{N} \int_{\mathbb{R}^{N}}|\nabla U|^{2} d x .
$$


For $s \in\left[m_{1}, m_{2}\right]$, let $S_{s}$ be the set of least energy solutions $U$ of (16) satisfying $U(0)=$ $\max _{x \in \mathbb{R}^{N}} U(x)$ and denote by $E_{s}$ the least energy level:

$$
E_{s}=\frac{1}{2} \int_{\mathbb{R}^{N}}\left[|\nabla U|^{2}+s U^{2}\right] d x-\int_{\mathbb{R}^{N}} F(U) d x, \quad U \in S_{s} .
$$

It is known from [25] and [7, Proposition 2.1] that for $s \in\left[m_{1}, m_{2}\right]$,

$$
E_{s}=\inf \left\{L_{s}(u) \mid G_{S}(u)=0, u \in H^{1}\left(\mathbb{R}^{N}\right) \backslash\{0\}\right\}
$$

and $E_{s}$ is attained by $u \in S_{S}$ and strictly increasing and continuous with respect to $s \in$ $\left[m_{1}, m_{2}\right]$.

For $s \in\left[m_{1}, m\right]$, we define $S_{s}^{m}$ to be the set of solutions $U$ of (16) satisfying $U(0)=$ $\max _{x \in \mathbb{R}^{N}} U(x)$ and $E_{s} \leq L_{s}(U) \leq E_{m}$. For $s \in\left[m, m_{2}\right]$, we define $S_{s}^{m}=S_{s}$. Since $f \in C^{1}$, it follows from the symmetry result in [21] that any solution of (16) is radially symmetric and decreasing with respect to $|x|$.

Proposition 1. For each $m^{\prime} \in\left[m_{1}, m\right]$ and $m^{\prime \prime} \in\left[m, m_{2}\right]$, the set $\bigcup_{s \in\left[m^{\prime}, m^{\prime \prime}\right]} S_{s}^{m}$ is compact. Moreover, there exist constants $C, c>0$ such that for any $U \in \bigcup_{s \in\left[m_{1}, m_{2}\right]}^{\left[m_{s}\right.} S_{s}^{m}$,

$$
U(x)+|\nabla U(x)| \leq C \exp (-c|x|) \quad \text { for all } x \in \mathbb{R}^{N} .
$$

Proof. By the identity (19), we see that $\bigcup_{s \in\left[m^{\prime}, m^{\prime \prime}\right]} S_{s}^{m}$ is bounded in $H^{1}\left(\mathbb{R}^{N}\right)$. Then, by the same argument as in [8], $\bigcup_{s \in\left[m^{\prime}, m^{\prime \prime}\right]} S_{s}^{m}$ is compact, and $\lim _{|x| \rightarrow \infty} U(x)=0$ uniformly for $U \in \bigcup_{s \in\left[m^{\prime}, m^{\prime \prime}\right]} S_{S}^{m}$. Then the decay property comes from the comparison principles.

Proposition 2. For any $a \in\left(m_{1}, m_{2}\right)$,

$$
\lim _{s \rightarrow a} \sup _{U \in S_{s}^{m}} \inf _{\tilde{U} \in S_{a}^{m}}\|U-\tilde{U}\|=0 .
$$

Proof. Suppose that the claim does not hold. Then there exist sequences $\left\{s_{l}\right\}_{l=1}^{\infty}$ with $\lim _{l \rightarrow \infty} s_{l}=a$ and $\left\{u_{l}\right\}_{l=1}^{\infty}$ with $u_{l} \in S_{s_{l}}^{m}$ such that $\left\{u_{l}\right\}_{l=1}^{\infty}$ is bounded away from $S_{a}^{m}$. From Proposition $1, u_{l}$ converges strongly up to a subsequence to some $u \in \bigcup_{s \in\left[a, m_{2}\right]} S_{s}^{m}$ in $H^{1}\left(\mathbb{R}^{N}\right)$ as $l \rightarrow \infty$. Note that $E_{s}$ is continuous with respect to $s \in\left[m_{1}, m_{2}\right]$. Since $\lim _{l \rightarrow \infty} s_{l}=a$, it follows that $u \in S_{a}^{m}$. This is a contradiction and proves the claim.

We recall that $A^{d}=\left\{x \in \mathbb{R}^{N} \mid \operatorname{dist}(x, A) \leq d\right\}$ for $A \subset \mathbb{R}^{N}$ and $d>0$. For a positive constant $d<\operatorname{dist}(\mathcal{M}, \partial O)$, we denote

$$
\underline{m}(d) \equiv \inf \left\{V(x) \mid x \in \mathcal{M}^{d}\right\} \quad \text { and } \quad \bar{m}(d) \equiv \sup \left\{V(x) \mid x \in \mathcal{M}^{d}\right\} .
$$

We note that $\lim _{d \rightarrow 0} \underline{m}(d)=\lim _{d \rightarrow 0} \bar{m}(d)=m$. From now on, we select a sufficiently small $d>0$ so that

$$
m_{1} \leq \underline{m}(d)<m<\bar{m}(d) \leq m_{2}
$$

and we define

$$
S \equiv \bigcup_{s \in[\underline{m}(d), \bar{m}(d)]} S_{s}^{m}
$$


We find a smooth radially symmetric function $\phi_{\varepsilon} \in C_{0}^{\infty}\left(\mathbb{R}^{N}\right)$ such that $\phi_{\varepsilon}(x)=1$ for $|x| \leq 1 / 2 \varepsilon^{1 / 3}, \phi_{\varepsilon}(x)=0$ for $|x| \geq 1 / \varepsilon^{1 / 3}$ and $\left|\nabla \phi_{\varepsilon}\right| \leq 3 \varepsilon^{1 / 3}$. Now we define

$$
\mathcal{Z}_{\varepsilon}^{10 \beta}=\left\{\phi_{\varepsilon}(\cdot-x / \varepsilon) U(\cdot-x / \varepsilon) \mid x \in \Omega^{10 \beta}, U \in S\right\} .
$$

Since $\Omega^{10 \beta} \subset \mathcal{M}^{d}$, the set $\mathcal{Z}_{\varepsilon}^{10 \beta}$ is well defined. By Proposition 1 , it is compact in $H_{\varepsilon}$. For $\delta>0$, we define

$$
N_{\delta}\left(\mathcal{Z}_{\varepsilon}^{10 \beta}\right) \equiv\left\{u \in H_{\varepsilon} \mid \operatorname{dist}_{\varepsilon}\left(u, \mathcal{Z}_{\varepsilon}^{10 \beta}\right) \leq \delta\right\}
$$

Here we use notation (14).

For some $\delta>0$, we will show that there exists a solution $u_{\varepsilon}$ of (3) in $N_{2 \delta}\left(\mathcal{Z}_{\varepsilon}^{10 \beta}\right)$ if $\varepsilon>0$ is sufficiently small. Then, for $N \geq 3$, it is standard to see from elliptic estimates (see [5, Proposition 3.5]) that there exists a large constant $K>t_{0}+1$ such that

$$
\left\|u_{\varepsilon}\right\|_{L^{\infty}} \leq K \quad \text { for all small } \varepsilon>0
$$

The constant $K$ depends only on the coefficient $C$ of (f2) and $p, N$ (see [5, Proposition 3.5]). Next for $N \geq 3$, we can find $\tilde{f} \in C^{1}(\mathbb{R})$ such that $\tilde{f}(t)=f(t)$ for $t \leq 2 K$, $\tilde{f}(t)=C t^{p}$ for $t \geq 3 K$ and $\tilde{f}$ satisfies conditions (f1)-(f3) with the same constants.

For $N=2$, from the Moser-Trudinger inequality and condition (f2), for any $q>1$, $r_{0}>0$ and $x_{0} \in \mathbb{R}^{N}$, we find a constant $\widetilde{K}\left(q, r_{0}\right)>0$ such that

$$
\left\|f\left(u_{\varepsilon}\right)\right\|_{L^{q}\left(B\left(x_{0}, 2 r_{0}\right)\right)} \leq \widetilde{K}\left(q, r_{0}\right) .
$$

Then, from the elliptic estimate in Theorem 8.17 of [22] and the fact $u_{\varepsilon} \in N_{2 \delta}\left(\mathcal{Z}_{\varepsilon}^{10 \beta}\right)$, we see that for $q>1$,

$$
\left\|u_{\varepsilon}\right\|_{L^{\infty}\left(B\left(x_{0}, r_{0}\right)\right)} \leq C\left(r_{0}\left\|u_{\varepsilon}\right\|_{L^{2}\left(B\left(x_{0}, 2 r_{0}\right)\right)}+\widetilde{K}\left(q, r_{0}\right)\right),
$$

where $C=C(q)$. Since $u_{\varepsilon} \in N_{2 \delta}\left(\mathcal{Z}_{\varepsilon}^{10 \beta}\right)$, there exists a constant $G>0$ such that $\left\|u_{\varepsilon}\right\|_{L^{2}\left(B\left(x_{0}, 2 r_{0}\right)\right)} \leq G$ for any $x_{0} \in \mathbb{R}^{N}$ and $r_{0}>0$. Consequently, (20) holds for large $K>t_{0}+1$. Next, for a sufficiently small $\alpha_{0}>0$, we can find $\tilde{f} \in C^{1}(\mathbb{R})$ and some constant $C_{\alpha_{0}}^{\prime}>0$ such that $\tilde{f}(t)=f(t)$ for $t \leq 2 K, \tilde{f}(t)=C_{\alpha_{0}}^{\prime} t^{4}$ for $t \geq 3 K$ and that $\tilde{f}(t) \leq C_{\alpha_{0}} \exp \left(\alpha_{0} t^{2}\right), t \geq 0$.

Thus for small $\varepsilon>0$, any solution $u_{\varepsilon} \in N_{\delta}\left(\mathcal{Z}_{\varepsilon}^{10 \beta}\right)$ of (2) with $\tilde{f}$ replacing $f$ satisfies the original equation (2). From now on, we can assume without loss of generality that the nonlinear function $f$ satisfies further

$$
\left|f^{\prime}(t) t\right| \leq C\left(1+t^{p}\right), \quad t \geq 0,
$$

where $p>1$ is given in (f2) for $N \geq 3$ and $p=4$ for $N=2$. 


\section{A center of mass for elements in $N_{2 \delta}\left(\mathcal{Z}_{\varepsilon}^{10 \beta}\right)$}

For $z \in \Omega_{\varepsilon}^{10 \beta} \equiv(1 / \varepsilon) \Omega^{10 \beta}$, we define

$$
S(z) \equiv\left\{\left(\phi_{\varepsilon} U\right)(\cdot-z) \mid U \in S\right\} .
$$

Then $\mathcal{Z}_{\varepsilon}^{10 \beta}=\bigcup_{z \in \Omega_{\varepsilon}^{10 \beta}} S(z)$. We can find $\xi>0$ such that for small $\varepsilon>0$ and $u \in \mathcal{Z}_{\varepsilon}^{10 \beta}$,

$$
\|u\|_{\varepsilon} \geq \xi .
$$

From the uniform decay property in Proposition 1, there exists $R_{0}>0$ such that for any $z_{1}, z_{2} \in \Omega_{\varepsilon}^{10 \beta}$ with $\left|z_{1}-z_{2}\right| \geq R_{0}$,

$$
\inf _{u_{1} \in S\left(z_{1}\right), u_{2} \in S\left(z_{2}\right)}\left\|u_{1}-u_{2}\right\|_{\varepsilon} \geq \xi / 2 \quad \text { if } \varepsilon>0 \text { is sufficiently small. }
$$

We take a small $\delta>0$ satisfying

$$
20 \delta \leq \xi .
$$

We pick $\sigma \in C_{0}^{\infty}(\mathbb{R},[0,1])$ such that $\sigma(x)=1$ for $|x| \leq 2 \delta$ and $\sigma(x)=0$ for $|x| \geq 3 \delta$. Then, for $u \in N_{2 \delta}\left(\mathcal{Z}_{\varepsilon}^{10 \beta}\right)$, we define the center of mass $\Upsilon_{\varepsilon}(u)$ of $u$ by

$$
\Upsilon_{\varepsilon}(u) \equiv \frac{\int_{\Omega_{\varepsilon}^{10 \beta}} \sigma\left(\operatorname{dist}_{\varepsilon}(u, S(z))\right) z d z}{\int_{\Omega_{\varepsilon}^{10 \beta}} \sigma\left(\operatorname{dist}_{\varepsilon}(u, S(z))\right) d z},
$$

where we use notation (14).

Then we have the following property.

Proposition 3. For $u \in N_{2 \delta}\left(\mathcal{Z}_{\varepsilon}^{10 \beta}\right)$, write $u=\phi_{\varepsilon}(\cdot-y) U(\cdot-y)+w$ with $U \in S(y), y \in$ $\Omega_{\varepsilon}^{10 \beta}$ and $\|w\|_{\varepsilon} \leq 2 \delta$. Then

$$
\left|\Upsilon_{\varepsilon}(u)-y\right| \leq R_{0} .
$$

Proof. Let $u=\phi_{\varepsilon}(\cdot-y) U(\cdot-y)+w$ with $\|w\|_{\varepsilon} \leq 2 \delta$. When $|z-y| \geq R_{0}$ and $v \in S(z)$, it follows from (21) and (22) that

$$
\|v-u\|_{\varepsilon} \geq\left\|v-\phi_{\varepsilon}(\cdot-y) U(\cdot-y)\right\|_{\varepsilon}-2 \delta \geq 10 \delta-2 \delta \geq 3 \delta .
$$

This implies that $\sigma\left(\operatorname{dist}_{\varepsilon}(u, S(z))\right)=0$ if $|z-y| \geq R_{0}$. Now the definition (23) of $\Upsilon_{\varepsilon}$ yields

$$
\left|\Upsilon_{\varepsilon}(u)-y\right|=\left|\frac{\int_{\Omega^{10 \beta}} \sigma\left(\operatorname{dist}_{\varepsilon}(u, S(z))\right)(z-y) d z}{\int_{\Omega^{10 \beta}} \sigma\left(\operatorname{dist}_{\varepsilon}(u, S(z))\right) d z}\right| \leq R_{0} .
$$

Proposition 4. For any $c \in(0,2]$, there exists $R_{1}>R_{0}$ such that for $u \in N_{c \delta}\left(\mathcal{Z}_{\varepsilon}^{10 \beta}\right)$,

$$
\int_{\left\{x \in \mathbb{R}^{N}|| x-\Upsilon_{\varepsilon}(u) \mid \geq R_{1}\right\}}\left[|\nabla u|^{2}+V_{\varepsilon} u^{2}\right] d x \leq(2 c \delta)^{2} .
$$

Proof. Let $u=\phi_{\varepsilon}(\cdot-y) U(\cdot-y)+w$ with $\|w\|_{\varepsilon} \leq c \delta$. From Proposition 3 and the decay property in Proposition 1, we see that

$$
\lim _{l \rightarrow \infty} \int_{\left\{x \in \mathbb{R}^{N}|| x-\Upsilon_{\varepsilon}(u) \mid \geq l\right\}}\left[\left|\nabla\left(\phi_{\varepsilon}(\cdot-y) U(\cdot-y)\right)\right|^{2}+V_{\varepsilon}\left(\phi_{\varepsilon}(\cdot-y) U(\cdot-y)\right)^{2}\right] d x=0
$$

uniformly for $y \in \Omega^{10 \beta}$ and $U \in S$. Then the claim follows from the fact $\|w\|_{\varepsilon} \leq c \delta$. 
5. A new neighborhood $G_{\delta}\left(\mathcal{Z}_{\varepsilon}^{10 \beta}\right)$ of $\mathcal{Z}_{\varepsilon}^{10 \beta}$

For $u \in N_{2 \delta}\left(\mathcal{Z}_{\varepsilon}^{10 \beta}\right)$ and $v \in H_{\varepsilon}$, we define

$$
|v|_{\varepsilon, u} \equiv \int_{\left|x-\Upsilon_{\varepsilon}(u)\right| \leq 1 / \sqrt{\varepsilon}}\left[|\nabla v|^{2}+V_{\varepsilon} v^{2}\right] d x .
$$

For $r \in(0,2 \delta]$, we define

$$
\begin{aligned}
G_{r}\left(\mathcal{Z}_{\varepsilon}^{10 \beta}\right) \equiv\left\{u \in N_{2 \delta}\left(\mathcal{Z}_{\varepsilon}^{10 \beta}\right) \mid\right. & \left|u-v_{z}\right|_{\varepsilon, u} \leq r^{2} / 2 \text { for some } z \in \Omega_{\varepsilon}^{10 \beta}, v_{z} \in S(z) \\
& \text { and } \left.\int_{\left|x-\Upsilon_{\varepsilon}(u)\right| \geq 1 / \sqrt{\varepsilon}}\left[|\nabla u|^{2}+V_{\varepsilon} u^{2}-2 F(u)\right] d x \leq r^{2} / 2\right\} .
\end{aligned}
$$

Proposition 5. There exists a constant $q=q(\delta)>0$ such that $\lim _{\delta \rightarrow 0} q(\delta)=0$, and for small $\varepsilon>0$ and $u \in N_{2 \delta}\left(\mathcal{Z}_{\varepsilon}^{10 \beta}\right)$,

$$
\begin{aligned}
(1-q) \int_{\left|x-\Upsilon_{\varepsilon}(u)\right| \geq 1 / \sqrt{\varepsilon}}\left[|\nabla u|^{2}\right. & \left.+V_{\varepsilon} u^{2}\right] d x \\
& \leq \int_{\left|x-\Upsilon_{\varepsilon}(u)\right| \geq 1 / \sqrt{\varepsilon}}\left[|\nabla u|^{2}+V_{\varepsilon} u^{2}-2 F(u)\right] d x
\end{aligned}
$$

and

$$
\begin{aligned}
(1+q) \int_{\left|x-\Upsilon_{\varepsilon}(u)\right| \geq 1 / \sqrt{\varepsilon}}\left[|\nabla u|^{2}\right. & \left.+V_{\varepsilon} u^{2}\right] d x \\
& \geq \int_{\left|x-\Upsilon_{\varepsilon}(u)\right| \geq 1 / \sqrt{\varepsilon}}\left[|\nabla u|^{2}+V_{\varepsilon} u^{2}-2 F(u)\right] d x .
\end{aligned}
$$

Proof. Note that for any small $c>0$, there exists $C>0$ such that $|F(t)| \leq c t^{2}+C t^{p+1}$ for some $p \in\left(1, \frac{N+2}{N-2}\right)$ when $N \geq 3$ and for $p=4$ when $N=2$. Thus,

$$
\int_{\left|x-\Upsilon_{\varepsilon}(u)\right| \geq 1 / \sqrt{\varepsilon}}|F(u)| d x \leq \int_{\left|x-\Upsilon_{\varepsilon}(u)\right| \geq 1 / \sqrt{\varepsilon}}\left[c u^{2}+C|u|^{p+1}\right] d x .
$$

From the Sobolev embedding theorem, there exists $C^{\prime}>0$, independent of small $\varepsilon>0$, such that

$$
\int_{\left|x-\Upsilon_{\varepsilon}(u)\right| \geq 1 / \sqrt{\varepsilon}}|u|^{p+1} d x \leq C^{\prime}\left(\int_{\left.\left|x-\Upsilon_{\varepsilon}(u)\right| \geq 1 / \sqrt{\varepsilon}\right\}}\left[|\nabla u|^{2}+V_{\varepsilon} u^{2}\right] d x\right)^{(p+1) / 2} .
$$

Then, for small $\varepsilon>0$, the inequalities (24) and (25) follow from Proposition 4.

Proposition 6. Let $c, c^{\prime} \in(0,1]$. Assume that $\delta>0$ is so small that the constant $q=$ $q(\delta)>0$ in Proposition 5 satisfies $q<\sqrt{2}-1$. Then, for small $\varepsilon>0$,

$$
G_{(1-q) c \delta}\left(\mathcal{Z}_{\varepsilon}^{10 \beta}\right) \subset N_{c \delta}\left(\mathcal{Z}_{\varepsilon}^{10 \beta}\right) \text { and } N_{c^{\prime} \delta}\left(\mathcal{Z}_{\varepsilon}^{10 \beta}\right) \subset G_{(1+q) \sqrt{2} c^{\prime} \delta}\left(\mathcal{Z}_{\varepsilon}^{10 \beta}\right) .
$$


Proof. If $u \in G_{(1-q) c \delta}\left(\mathcal{Z}_{\varepsilon}^{10 \beta}\right)$, it follows from (24) that

$$
\int_{\left|x-\Upsilon_{\varepsilon}(u)\right| \geq 1 / \sqrt{\varepsilon}}\left[|\nabla u|^{2}+V_{\varepsilon} u^{2}\right] d x \leq(1-q)(c \delta)^{2} / 2 .
$$

Note that for some $z \in \Omega_{\varepsilon}^{10 \beta}$ and $v_{z} \in S(z)$,

$$
\int_{\left|x-\Upsilon_{\varepsilon}(u)\right| \leq 1 / \sqrt{\varepsilon}}\left[\left|\nabla\left(u-v_{z}\right)\right|^{2}+V_{\varepsilon}\left(u-v_{z}\right)^{2}\right] d x \leq((1-q) c \delta)^{2} / 2 .
$$

From (21), (22) and Proposition 3, we see that $\left|\Upsilon_{\varepsilon}(u)-z\right|$ is bounded for small $\varepsilon>0$. Then, from the decay property of $v_{z} \in S(z)$ in Proposition 1, we see that

$$
\lim _{\varepsilon \rightarrow 0} \int_{\left|x-\Upsilon_{\varepsilon}(u)\right| \geq 1 / \sqrt{\varepsilon}}\left[\left|\nabla v_{z}\right|^{2}+V_{\varepsilon}\left(v_{z}\right)^{2}\right] d x=0 .
$$

It follows that for small $\varepsilon>0$,

$$
\begin{aligned}
\left\|u-v_{z}\right\|_{\varepsilon}^{2}= & \int_{\left|x-\Upsilon_{\varepsilon}(u)\right| \leq 1 / \sqrt{\varepsilon}}\left[\left|\nabla\left(u-v_{z}\right)\right|^{2}+V_{\varepsilon}\left(u-v_{z}\right)^{2}\right] d x \\
& +\int_{\left|x-\Upsilon_{\varepsilon}(u)\right| \geq 1 / \sqrt{\varepsilon}}\left[\left|\nabla\left(u-v_{z}\right)\right|^{2}+V_{\varepsilon}\left(u-v_{z}\right)^{2}\right] d x \\
\leq & (c \delta)^{2} / 2+(c \delta)^{2} / 2=(c \delta)^{2} .
\end{aligned}
$$

This proves the first inclusion in (26).

If $u \in N_{c^{\prime} \delta}\left(\mathcal{Z}_{\varepsilon}^{10 \beta}\right)$, it follows that

$$
\left\|u-\phi_{\varepsilon}(\cdot-z) U(\cdot-z)\right\|_{\varepsilon}^{2} \leq\left(c^{\prime} \delta\right)^{2} \quad \text { for some } z \in \Omega_{\varepsilon}^{10 \beta}, U \in S .
$$

From Proposition 3 and the decay property of $U$ in Proposition 1, we see that

$$
\lim _{\varepsilon \rightarrow 0} \int_{\left|x-\Upsilon_{\varepsilon}(u)\right| \geq 1 / \sqrt{\varepsilon}}\left[\left|\nabla\left(\phi_{\varepsilon}(\cdot-z) U(\cdot-z)\right)\right|^{2}+V_{\varepsilon}\left(\phi_{\varepsilon}(\cdot-z) U(\cdot-z)\right)^{2}\right] d x=0 .
$$

Then it follows that for small $\varepsilon>0,\left|u-\phi_{\varepsilon}(\cdot-z) U(\cdot-z)\right|_{\varepsilon, u} \leq\left(c^{\prime} \delta\right)^{2}$ and

$$
\int_{\left|x-\Upsilon_{\varepsilon}(u)\right| \geq 1 / \sqrt{\varepsilon}}\left[|\nabla u|^{2}+V_{\varepsilon} u^{2}\right] d x \leq(1+q)\left(c^{\prime} \delta\right)^{2} .
$$

From (25), we see that

$$
\int_{\left|x-\Upsilon_{\varepsilon}(u)\right| \geq 1 / \sqrt{\varepsilon}}\left[|\nabla u|^{2}+V_{\varepsilon} u^{2}-2 F(u)\right] d x \leq\left((1+q) c^{\prime} \delta\right)^{2} .
$$

This implies that $u \in G_{(1+q) \sqrt{2} c^{\prime} \delta}\left(\mathcal{Z}_{\varepsilon}^{10 \beta}\right)$, and proves the second inclusion in (26). 


\section{A starting surface and its energy estimate}

From condition (V2), we can find a manifold $L_{\varepsilon} \in \mathcal{L}\left(L_{0}\right)$ and a map $\gamma_{\varepsilon} \in \Lambda_{L_{\varepsilon}} \subset$ $C\left(L_{\varepsilon}, O\right)$ such that $V\left(\gamma_{\varepsilon}(z)\right) \leq m+\varepsilon$ for any $z \in L_{\varepsilon}$. We may assume that $\partial L_{\varepsilon}=L_{0}$ and $\gamma_{\varepsilon}(z)=z$ for $z \in L_{0}$. We choose any positive number $\bar{\alpha}<\left(m-m_{0}\right) / 2$. Then, if $\varepsilon>0$ is sufficiently small, by condition (V3), there exist $\alpha_{0} \in(0, \bar{\alpha})$ and a continuous map $\Phi:[0,1] \times O \rightarrow O$ such that for small $\varepsilon>0$,

$$
\begin{gathered}
\Phi\left(1, \gamma_{\varepsilon}(z)\right)=\Phi(1, z)=z \quad \text { for } z \in L_{0}, \\
V\left(\Phi\left(1, \gamma_{\varepsilon}(z)\right)\right) \leq m-\alpha_{0} \quad \text { for } \Phi\left(1, \gamma_{\varepsilon}(z)\right) \notin \Omega^{\beta} \\
V\left(\Phi\left(1, \gamma_{\varepsilon}(z)\right)\right) \leq m+\varepsilon \quad \text { for any } z \in L_{\varepsilon} .
\end{gathered}
$$

We define a starting surface $\Theta_{\varepsilon}(z) \equiv \Phi\left(1, \gamma_{\varepsilon}(z)\right), z \in L_{\varepsilon}$. We note that $\Theta_{\varepsilon}(z)=z$ for $z \in L_{0}$. Now, for $N \geq 3$, taking a solution $U \in S_{m}$, we define a map $A_{\varepsilon}:(0, \infty) \times L_{\varepsilon} \rightarrow$ $H_{\varepsilon}$ by

$$
A_{\varepsilon}(t, z)(x)=\phi_{\varepsilon}\left(x / t-\Theta_{\varepsilon}(z) / t \varepsilon\right) U\left(x / t-\Theta_{\varepsilon}(z) / t \varepsilon\right) .
$$

We can find a large $T>1$ such that $\Gamma_{\varepsilon}\left(A_{\varepsilon}(T, z)\right)<-1$ for small $\varepsilon>0$ and any $z \in L_{\varepsilon}$. We define $A_{\varepsilon}(0, z)=0$. Then we see that $A_{\varepsilon}:[0, T] \times L_{\varepsilon} \rightarrow H_{\varepsilon}$ is continuous.

For $N=2$, we need to take more involved operations on $\phi_{\varepsilon} U$ as in [9]. We recall some facts from [9]. For a fixed $U \in S_{m}$, we define $g(\theta, s):(0, \infty) \times(0, \infty) \rightarrow \mathbb{R}$ by

$$
g(\theta, s)=L_{m}(\theta U(\cdot / s))=\frac{\theta^{2}}{2}\|\nabla U\|_{L^{2}}^{2}+s^{2} \int_{\mathbb{R}^{2}}\left[\frac{1}{2}(\theta U)^{2}-F(\theta U)\right] d x .
$$

For any small $\iota>0$ and $\theta_{0} \in(0,1), s_{0} \in(0,1-\iota)$, let $\zeta(t)=(\theta(t), s(t)):[0, \infty) \rightarrow \mathbb{R}^{2}$ be a piecewise linear curve joining

$$
\begin{aligned}
\left(0, s_{0}\right) \rightarrow\left(1-\theta_{0}, s_{0}\right) \rightarrow\left(1-\theta_{0}, 1-\iota\right) \rightarrow(1,1-\iota) \rightarrow(1,1) & \rightarrow(1,1+\iota) \\
\rightarrow\left(1+\theta_{0}, 1+\iota\right) & \rightarrow\left(1+\theta_{0}, \infty\right)
\end{aligned}
$$

Let $0 \equiv t_{0}<t_{1}<\cdots<t_{6}<t_{7} \equiv \infty$ be such that for each $i=0, \ldots, 7, \zeta\left(t_{i}\right)$ is an end point of a linear segment of the piecewise linear curve $\zeta$. We set

$$
U_{t}(x)=\theta(t) U(x / s(t)) .
$$

Then, for any small $\iota>0$, there exist $\theta_{0} \in(0,1)$ and $s_{0} \in(0,1-\iota)$ such that for any small $\iota>0$, the function $t \mapsto L_{m}\left(U_{t}\right)=g(\zeta(t))$ is strictly increasing on $\left(t_{0}, t_{3}\right)$, constant on $\left(t_{3}, t_{5}\right)$, strictly decreasing on $\left(t_{5}, t_{7}\right)$ and $\lim _{t \rightarrow \infty} L_{m}\left(U_{t}\right)=-\infty$. Then we define a map $A_{\varepsilon}:(0, \infty) \times L_{\varepsilon} \rightarrow H_{\varepsilon}$ by

$$
A_{\varepsilon}(t, z)(x)=\phi_{\varepsilon}\left(x / s(t)-\Theta_{\varepsilon}(z) / s(t) \varepsilon\right) \theta(t) U\left(x / s(t)-\Theta_{\varepsilon}(z) / s(t) \varepsilon\right) .
$$


Proposition 7. We have

$$
\lim _{\varepsilon \rightarrow 0} \max _{t \in[0, T], z \in L_{\varepsilon}} \Gamma_{\varepsilon}\left(A_{\varepsilon}(t, z)\right) \leq E_{m} .
$$

Moreover, for any small $v \in(0,1)$, taking $\iota \in(0, v / 2)$ if $N=2$, we see that

$$
\limsup _{\varepsilon \rightarrow 0} \max \left\{\Gamma_{\varepsilon}\left(A_{\varepsilon}(t, z)\right) \mid t \in[0, T] \backslash(1-v, 1+v), z \in L_{\varepsilon}\right\}<E_{m},
$$

and

$$
\limsup _{\varepsilon \rightarrow 0} \max \left\{\Gamma_{\varepsilon}\left(A_{\varepsilon}(t, z)\right) \mid t \in[0, T], z \in L_{\varepsilon}, \Theta_{\varepsilon}(z) \notin \Omega^{\beta}\right\}<E_{m} .
$$

Proof. First we consider the case $N \geq 3$. Then

$$
\begin{aligned}
& \Gamma_{\varepsilon}\left(A_{\varepsilon}(t, z)\right) \\
& \quad=\frac{1}{2} \int_{\mathbb{R}^{N}}\left[t^{N-2}\left|\nabla\left(\phi_{\varepsilon} U\right)\right|^{2}+t^{N} V\left(\varepsilon t x+\Theta_{\varepsilon}(z)\right)\left(\phi_{\varepsilon} U\right)^{2}\right] d x-\int_{\mathbb{R}^{N}} t^{N} F\left(\phi_{\varepsilon} U\right) d x .
\end{aligned}
$$

Then, from (28) and the exponential decay of $U$ in Proposition 1,

$$
\begin{aligned}
\Gamma_{\varepsilon}\left(A_{\varepsilon}(t, z)\right) & =\frac{1}{2} \int_{\mathbb{R}^{N}}\left[t^{N-2}|\nabla U|^{2}+t^{N} V\left(\Theta_{\varepsilon}(z)\right) U^{2}\right] d x-\int_{\mathbb{R}^{N}} t^{N} F(U) d x+o(1) \\
& \leq \frac{1}{2} \int_{\mathbb{R}^{N}}\left[t^{N-2}|\nabla U|^{2}+t^{N} m U^{2}\right] d x-\int_{\mathbb{R}^{N}} t^{N} F(U) d x+o(1)
\end{aligned}
$$

uniformly for $z \in L_{\varepsilon}$ as $\varepsilon \rightarrow 0$. We see from the Pohozaev identity (18) that the function

$$
g(t) \equiv \frac{1}{2} \int_{\mathbb{R}^{N}}\left[t^{N-2}|\nabla U|^{2}+t^{N} m U^{2}\right] d x-\int_{\mathbb{R}^{N}} t^{N} F(U) d x
$$

satisfies

$$
\begin{aligned}
g(t) \leq g(1) & \text { for } t \in[0, T], \\
g^{\prime}(t)(t-1)<0 & \text { for } t \in(0, T) \backslash\{0\} .
\end{aligned}
$$

Thus, we get the first and second estimates.

For the third estimate, we note from (27), (28) and the above estimates that when $\Theta_{\varepsilon} \notin \Omega^{\beta}$,

$$
\begin{aligned}
\Gamma_{\varepsilon}\left(A_{\varepsilon}(t, z)\right) & =\frac{1}{2} \int_{\mathbb{R}^{N}}\left[t^{N-2}|\nabla U|^{2}+t^{N} V\left(\Theta_{\varepsilon}(z)\right) U^{2}\right] d x-\int_{\mathbb{R}^{N}} t^{N} F(U) d x+o(1) \\
& \leq \frac{1}{2} \int_{\mathbb{R}^{N}}\left[t^{N-2}|\nabla U|^{2}+t^{N}\left(m-\alpha_{0}\right) U^{2}\right] d x-\int_{\mathbb{R}^{N}} t^{N} F(U) d x+o(1) \\
& =g(t)-\frac{t^{N} \alpha_{0}}{2} \int_{\mathbb{R}^{N}} U^{2} d x+o(1) .
\end{aligned}
$$


It is easy to see that

$$
g(t)-\frac{t^{n} \alpha_{0}}{2} \int_{\mathbb{R}^{N}} U^{2} d x \leq \max \left\{g(1 / 2), g(1)-\frac{\alpha_{0}}{2^{N+1}} \int_{\mathbb{R}^{N}} U^{2} d x\right\} .
$$

This proves the third estimate, and completes the proof for $N \geq 3$.

For $N=2$, we see that

$$
\begin{aligned}
& \Gamma_{\varepsilon}\left(A_{\varepsilon}(t, z)\right) \\
&=\frac{1}{2} \int_{\mathbb{R}^{N}}\left[(\theta(t))^{2}|\nabla U|^{2}+(s(t))^{2} V\left(\Theta_{\varepsilon}(z)\right)(\theta(t) U)^{2}\right] d x \\
& \quad-\int_{\mathbb{R}^{N}}(s(t))^{2} F(\theta(t) U) d x+o(1) \\
& \leq \frac{1}{2} \int_{\mathbb{R}^{N}}\left[(\theta(t))^{2}|\nabla U|^{2}+(s(t))^{2} m(\theta(t) U)^{2}\right] d x-\int_{\mathbb{R}^{N}}(s(t))^{2} F(\theta(t) U) d x+o(1) \\
&= L_{m}\left(U_{t}\right)+o(1)
\end{aligned}
$$

uniformly for $z \in L_{\varepsilon}$ as $\varepsilon \rightarrow 0$. Now the claim follows from the construction $U_{t}$ for $N=2$.

\section{Gradient estimate}

Now we define

$$
C_{\varepsilon}=\max _{t \in[0, T], z \in L_{\varepsilon}} \Gamma_{\varepsilon}\left(A_{\varepsilon}(t, z)\right) .
$$

Proposition 8. For sufficiently small $\delta>0$ and any $\delta^{\prime} \in(0, \delta)$, there exists $\mu=$ $\mu\left(\delta, \delta^{\prime}\right)>0$, independent of small $\varepsilon>0$, such that

$$
\inf \left\{\left\|\Gamma_{\varepsilon}^{\prime}(u)\right\|_{\varepsilon}^{*} \mid u \in \Gamma_{\varepsilon}^{C_{\varepsilon}} \cap\left(G_{2 \delta}\left(\mathcal{Z}_{\varepsilon}^{10 \beta}\right) \backslash G_{\delta^{\prime}}\left(\mathcal{Z}_{\varepsilon}^{10 \beta}\right)\right), \Upsilon_{\varepsilon}(u) \in \Omega_{\varepsilon}^{9 \beta}\right\} \geq \mu\left(\delta, \delta^{\prime}\right) .
$$

Proof. To the contrary, suppose that for some $\delta^{\prime} \in(0, \delta)$, there exists an element $u_{\varepsilon} \in$ $\Gamma_{\varepsilon}^{C_{\varepsilon}} \cap\left(G_{2 \delta}\left(\mathcal{Z}_{\varepsilon}^{10 \beta}\right) \backslash G_{\delta^{\prime}}\left(\mathcal{Z}_{\varepsilon}^{10 \beta}\right)\right)$ with $\Upsilon_{\varepsilon}(u) \in \Omega_{\varepsilon}^{9 \beta}$ such that $\Gamma_{\varepsilon}^{\prime}\left(u_{\varepsilon}\right)$ converges to 0 , up to a subsequence, as $\varepsilon \rightarrow 0$. Since $u_{\varepsilon} \in \Gamma_{\varepsilon}^{C_{\varepsilon}} \cap G_{2 \delta}\left(\mathcal{Z}_{\varepsilon}^{10 \beta}\right)$ and $\Upsilon_{\varepsilon}\left(u_{\varepsilon}\right) \in \Omega_{\varepsilon}^{9 \beta}$, from Proposition 3 there exists $x_{\varepsilon} \in \mathbb{R}^{N}$ with $\operatorname{dist}\left(x_{\varepsilon}, \Omega^{9 \beta}\right) \leq \varepsilon R_{0}$ such that $u_{\varepsilon}=$ $\phi_{\varepsilon}\left(\cdot-x_{\varepsilon} / \varepsilon\right) U_{\varepsilon}\left(\cdot-x_{\varepsilon} / \varepsilon\right)+w_{\varepsilon}$ for some $U_{\varepsilon} \in S$ and some $w_{\varepsilon} \in H_{\varepsilon}$ with $\left\|w_{\varepsilon}\right\|_{\varepsilon} \leq 2 \delta$.

Suppose there exist $y_{\varepsilon} \in B\left(x_{\varepsilon} / \varepsilon, 2 / \varepsilon^{1 / 3}\right) \backslash B\left(x_{\varepsilon} / \varepsilon, 1 / 4 \varepsilon^{1 / 3}\right)$ and $R>0$ satisfying $\liminf _{\varepsilon \rightarrow 0} \int_{B\left(y_{\varepsilon}, R\right)} u_{\varepsilon}^{2} d y>0$. Taking a subsequence, we can assume that as $\varepsilon \rightarrow 0, \varepsilon y_{\varepsilon}$ converges to some $x_{0}$ in the closure of $\Omega^{9 \beta}$ and $u_{\varepsilon}\left(\cdot+y_{\varepsilon}\right) \rightarrow \tilde{W}$ weakly in $H^{1}\left(\mathbb{R}^{N}\right)$ for some $\tilde{W} \in H^{1}\left(\mathbb{R}^{N}\right) \backslash\{0\}$. Then $\tilde{W}$ satisfies

$$
\Delta \tilde{W}(y)-V\left(x_{0}\right) \tilde{W}(y)+f(\tilde{W}(y))=0 \quad \text { for } y \in \mathbb{R}^{N} .
$$

By definition, $L_{V\left(x_{0}\right)}(\tilde{W}) \geq E_{V\left(x_{0}\right)}$. Also, for large $R>0$,

$$
\liminf _{\varepsilon \rightarrow 0} \int_{B\left(y_{\varepsilon}, R\right)}\left|\nabla u_{\varepsilon}\right|^{2} d y \geq \frac{1}{2} \int_{\mathbb{R}^{N}}|\nabla \tilde{W}|^{2} d y .
$$


Since $V\left(x_{0}\right) \geq \underline{m}(d)$, we see from (19) and (30) that

$$
\liminf _{\varepsilon \rightarrow 0} \int_{B\left(x_{\varepsilon}, R\right)}\left|\nabla u_{\varepsilon}\right|^{2} d y \geq \frac{N}{2} L_{V\left(x_{0}\right)}(\tilde{W}) \geq \frac{N}{2} E_{\underline{m}(d)}>0 .
$$

Then, taking $\delta \in\left(0, \sqrt{(N / 4) E_{\underline{m}(d)}}\right)$, we get a contradiction. Since there does not exist such a sequence $\left\{y_{\varepsilon}\right\}_{\varepsilon} \in B\left(x_{\varepsilon} / \varepsilon, 2 / \varepsilon^{1 / 3}\right) \backslash B\left(x_{\varepsilon} / \varepsilon, 1 / 4 \varepsilon^{1 / 3}\right)$ and

$$
\operatorname{supp}\left(\nabla \phi_{\varepsilon}\left(\cdot-x_{\varepsilon} / \varepsilon\right)\right)=B\left(x_{\varepsilon} / \varepsilon, 1 / \varepsilon^{1 / 3}\right) \backslash B\left(x_{\varepsilon} / \varepsilon, 1 / 2 \varepsilon^{1 / 3}\right),
$$

we deduce from a result of P.-L. Lions (see [29, Lemma I.1]) that for $N \geq 3$,

$$
\liminf _{\varepsilon \rightarrow 0} \int_{\operatorname{supp}\left(\left|\nabla \phi_{\varepsilon}\left(-x_{\varepsilon} / \varepsilon\right)\right|\right)}\left|u_{\varepsilon}\right|^{p+1} d y=0 .
$$

For $N=2$, we see from [9, Lemma 1] that $\lim _{\varepsilon \rightarrow \infty} \int_{\operatorname{supp}\left(\left|\nabla \phi_{\varepsilon}\left(-x_{\varepsilon} / \varepsilon\right)\right|\right)} G\left(u_{\varepsilon}\right) d x=0$ for any $G \in C\left(\mathbb{R}, \mathbb{R}\right.$ ) satisfying (i) $\lim _{t \rightarrow 0} G(t) / t^{2}=0$ and (ii) for any $\alpha>0$ there exists $C_{\alpha}>0$ such that $|G(t)| \leq C_{\alpha} e^{\alpha t^{2}}$ for all $t \in \mathbb{R}$. As a consequence, we can derive using (f1), (f2) and boundedness of $\left\{\left\|u_{\varepsilon}\right\|_{L^{2}}\right\}_{\varepsilon}$ that for $\hat{u}_{\varepsilon} \equiv\left(1-\phi_{\varepsilon}\left(\cdot-x_{\varepsilon} / \varepsilon\right)\right)^{2} u_{\varepsilon}$,

$$
\lim _{\varepsilon \rightarrow 0} \int_{\mathbb{R}^{N}}\left(f\left(u_{\varepsilon}\right) \hat{u}_{\varepsilon}-f\left(\hat{u}_{\varepsilon}\right) \hat{u}_{\varepsilon}\right) d y=0
$$

Note that

$$
\Gamma_{\varepsilon}^{\prime}\left(u_{\varepsilon}\right) \hat{u}_{\varepsilon}=\int_{\mathbb{R}^{N}}\left[\left|\nabla \hat{u}_{\varepsilon}\right|^{2}-u_{\varepsilon}^{2}\left|\nabla \phi_{\varepsilon}\left(\cdot-x_{\varepsilon} / \varepsilon\right)\right|^{2}+V_{\varepsilon} \hat{u}_{\varepsilon}^{2}-f\left(u_{\varepsilon}\right) \hat{u}_{\varepsilon}\right] d y .
$$

Since $\left|\nabla \phi_{\varepsilon}\left(\cdot-x_{\varepsilon} / \varepsilon\right)\right| \leq 3 \varepsilon^{1 / 3}$, we see that

$$
0=\lim _{\varepsilon \rightarrow 0} \Gamma_{\varepsilon}^{\prime}\left(u_{\varepsilon}\right) \hat{u}_{\varepsilon}=\lim _{\varepsilon \rightarrow 0} \int_{\mathbb{R}^{N}}\left[\left|\nabla \hat{u}_{\varepsilon}\right|^{2}+V_{\varepsilon} \hat{u}_{\varepsilon}^{2}-f\left(\hat{u}_{\varepsilon}\right) \hat{u}_{\varepsilon}\right] d y .
$$

We note that $\left\|\hat{u}_{\varepsilon}\right\|_{\varepsilon} \leq 3 \delta$ for small $\varepsilon>0$. Then, for $N \geq 3$, it follows from Sobolev's inequality that for some $C, c>0$,

$$
\begin{aligned}
\int_{\mathbb{R}^{N}}\left[\left|\nabla \hat{u}_{\varepsilon}\right|^{2}+V_{\varepsilon} \hat{u}_{\varepsilon}^{2}-f\left(\hat{u}_{\varepsilon}\right) \hat{u}_{\varepsilon}\right] d y & \geq\left\|\hat{u}_{\varepsilon}\right\|_{\varepsilon}^{2}-\frac{V_{0}}{2} \int_{\mathbb{R}^{N}} \hat{u}_{\varepsilon}^{2} d y-C \int_{\mathbb{R}^{N}}\left(\hat{u}_{\varepsilon}\right)^{2 N /(N-2)} d y \\
& \geq \frac{1}{2}\left\|\hat{u}_{\varepsilon}\right\|_{\varepsilon}^{2}-C c\left\|\hat{u}_{\varepsilon}\right\|_{\varepsilon}^{2 N /(N-2)} \\
& \geq\left\|\hat{u}_{\varepsilon}\right\|_{\varepsilon}^{2}\left(\frac{1}{2}-C c(3 \delta)^{4 /(N-2)}\right)
\end{aligned}
$$

Taking $\delta>0$ small enough, we see from (32) and (33) that

$$
\lim _{\varepsilon \rightarrow 0}\left\|\hat{u}_{\varepsilon}\right\|_{\varepsilon}=0
$$

For $N=2$, recalling $f(t)=C_{\alpha_{0}}^{\prime} t^{4}$ for $t \geq 3 K$, we can argue in a similar way.

We define $v_{\varepsilon}(x) \equiv u_{\varepsilon}\left(x+x_{\varepsilon} / \varepsilon\right)$. Then $v_{\varepsilon}$ converges weakly to some $W$ in $H^{1}$ as $\varepsilon \rightarrow 0$ after extracting a subsequence. 
We may assume that $x_{\varepsilon}$ converges to a point $x_{0} \in \Omega^{10 \beta}$ as $\varepsilon \rightarrow 0$. Then

$$
\Delta W-V\left(x_{0}\right) W+f(W)=0 \quad \text { in } \mathbb{R}^{N} .
$$

Just as before, for $z_{\varepsilon} \in \mathbb{R}^{N}$ satisfying $\lim _{\varepsilon \rightarrow 0}\left|z_{\varepsilon}-x_{\varepsilon} / \varepsilon\right|=\infty$, and $R>0$,

$$
\lim _{\varepsilon \rightarrow 0} \int_{B\left(z_{\varepsilon}, R\right)} u_{\varepsilon}^{2} d x=0 .
$$

This and (34) imply that

$$
\lim _{\varepsilon \rightarrow 0} \int_{\mathbb{R}^{N}} f\left(u_{\varepsilon}\right) u_{\varepsilon} d x=\int_{\mathbb{R}^{N}} f(W) W d x, \quad \lim _{\varepsilon \rightarrow 0} \int_{\mathbb{R}^{N}} F\left(u_{\varepsilon}\right) d x=\int_{\mathbb{R}^{N}} F(W) d x .
$$

Thus we see that

$$
\lim _{\varepsilon \rightarrow 0} \int_{\mathbb{R}^{N}}\left[\left|\nabla u_{\varepsilon}\right|^{2}+V_{\varepsilon} u_{\varepsilon}^{2}\right] d x=\int_{\mathbb{R}^{N}}\left[|\nabla W|^{2}+V\left(x_{0}\right) W^{2}\right] d x,
$$

and $L_{V\left(x_{0}\right)}(W) \leq E_{m}$; it follows that for some $y_{0} \in \mathbb{R}^{N}$,

$$
\tilde{W} \equiv W\left(\cdot+y_{0}\right) \in S .
$$

Note that $\lim _{\varepsilon \rightarrow 0} V(\varepsilon x)=V\left(x_{0}\right)$ uniformly on $B\left(x_{\varepsilon} / \varepsilon, 1 / \varepsilon^{1 / 3}\right)$. Now, we see from (34) that

$$
\lim _{\varepsilon \rightarrow 0}\left\|u_{\varepsilon}-\left(\phi_{\varepsilon} \tilde{W}\right)\left(\cdot-y_{0}-x_{\varepsilon} / \varepsilon\right)\right\|_{\varepsilon}=0 .
$$

This implies that for small $\varepsilon>0, u_{\varepsilon} \in G_{\delta^{\prime}}\left(\mathcal{Z}_{\varepsilon}^{10 \beta}\right)$ for small $\varepsilon>0$. This is a contradiction, and completes the proof.

\section{An energy decreasing deformation for tails}

For $u \in H_{\varepsilon}$, we suppose that

$$
\int_{\mathbb{R}^{N} \backslash B(y, R)}\left[|\nabla u|^{2}+V_{\varepsilon} u^{2}\right] d x \leq b^{2} / 2 .
$$

We consider the following minimization problem:

$$
I_{y, b}^{R}(u) \equiv \inf \left\{\int_{\mathbb{R}^{N} \backslash B(y, R)}\left[\frac{1}{2}\left(|\nabla v|^{2}+V_{\varepsilon} v^{2}\right)-F(v)\right] d x \mid v \in H_{y, b}^{R}(u)\right\},
$$

where

$$
H_{y, b}^{R}(u) \equiv\left\{v \in H_{\varepsilon} \mid \int_{\mathbb{R}^{N} \backslash B(y, R)}\left[|\nabla v|^{2}+V_{\varepsilon} v^{2}\right] d x \leq b^{2}, v=u \text { on } B(y, R)\right\} .
$$


Proposition 9. Assume (35) holds with sufficiently small $b>0$. Then there exists $a$ unique minimizer $v$ of $I_{y, b}^{R}(u)$ in $H_{y, b}^{R}(u)$ which satisfies $\int_{\mathbb{R}^{N} \backslash B(y, R)}\left(|\nabla v|^{2}+V_{\varepsilon} v^{2}\right) d x$ $<b^{2}$, and

$$
\Delta v-V_{\varepsilon} v+f(v)=0 \quad \text { in } \mathbb{R}^{N} \backslash B(0, R), \quad v=u \text { in } B(y, R) .
$$

Moreover, there exist $c, C>0$, independent of small $\varepsilon>0$, such that

$$
v(x) \leq C \exp (-c(|x-y|-R-1)) \quad \text { for }|x-y| \geq R+1 .
$$

Proof. Since $f^{\prime}(0)=0$ and the growth condition (f2) holds, it is rather standard to show that if $b>0$ is sufficiently small, then

$$
\int_{\mathbb{R}^{N} \backslash B(y, R)}\left[\frac{1}{2}\left(|\nabla u|^{2}+V_{\varepsilon} u^{2}\right)-F(u)\right] d x<3 b^{2} / 8,
$$

and for $v \in H_{y, \delta}^{R}(u)$ with $\int_{\mathbb{R}^{N} \backslash B(y, R)}\left(|\nabla v|^{2}+V_{\varepsilon} v^{2}\right) d x=b^{2}$,

$$
\int_{\mathbb{R}^{N} \backslash B(y, R)}\left[\frac{1}{2}\left(|\nabla v|^{2}+V_{\varepsilon} v^{2}\right)-F(v)\right] d x>3 b^{2} / 8 .
$$

Then, as in [12] and [6], we see that there exists a unique minimizer $v(u, y, R)$ of $I_{y, b}^{R}(u)$ with $\int_{\mathbb{R}^{N} \backslash B(y, R)}\left(\left|\nabla v_{\varepsilon}\right|^{2}+V_{\varepsilon}\left(v_{\varepsilon}\right)^{2}\right) d x<b^{2}$. For details, see Proposition 5.7 of [12] and Proposition 2.3 of [6].

In what follows, we denote the unique minimizer of $I_{y, b}^{R}(u)$ in $H_{y, b}^{R}(u)$ by $v(u, y, R)$.

From Proposition 3, we see that for any $u \in G_{2 \delta}\left(\mathcal{Z}_{\varepsilon}^{10 \beta}\right)$, there exists $y \in \mathbb{R}^{N}$ satisfying $\left|y-\Upsilon_{\varepsilon}(u)\right| \leq R_{0}$ and $\left\|u-\phi_{\varepsilon}(\cdot-y) U(\cdot-y)\right\|_{\varepsilon} \leq 2 \delta$. Then, for small $\varepsilon>0$,

$$
\operatorname{supp}\left(\phi_{\varepsilon}(\cdot-y) U(\cdot-y)\right) \subset B\left(\Upsilon_{\varepsilon}(u), 1 / \sqrt{\varepsilon}\right) .
$$

Thus, it follows that for any $u \in G_{2 \delta}\left(\mathcal{Z}_{\varepsilon}^{10 \beta}\right)$,

$$
\int_{\mathbb{R}^{N} \backslash B\left(\Upsilon_{\varepsilon}(u), 1 / \sqrt{\varepsilon}\right)}\left(|\nabla u|^{2}+V_{\varepsilon} u^{2}\right) d x \leq(2 \delta)^{2} .
$$

We take a small $\delta>0$ so that Proposition 9 holds for $b=2 \sqrt{2} \delta$. Then it is important to see from the definition of the new neighborhood $G_{2 \delta}\left(\mathcal{Z}_{\varepsilon}^{10 \beta}\right)$ that $v\left(u, \Upsilon_{\varepsilon}(u), 1 / \sqrt{\varepsilon}\right) \in$ $G_{2 \delta}\left(\mathcal{Z}_{\varepsilon}^{10 \beta}\right)$ for $u \in G_{2 \delta}\left(\mathcal{Z}_{\varepsilon}^{10 \beta}\right)$. We define

$$
\tau_{\varepsilon}(u) \equiv v\left(u, \Upsilon_{\varepsilon}(u), 1 / \sqrt{\varepsilon}\right) \quad \text { for } u \in G_{2 \delta}\left(\mathcal{Z}_{\varepsilon}^{10 \beta}\right) .
$$

From the uniqueness of a minimizer of $I_{\Upsilon_{\varepsilon}(u), 2 \sqrt{2} \delta}^{R}(u)$, we see that $\tau_{\varepsilon}$ is continuous on $G_{2 \delta}\left(\mathcal{Z}_{\varepsilon}^{10 \beta}\right)$. We also see from the definition $\tau_{\varepsilon}(u)$ that for any $c \in(0,1]$,

$$
\tau_{\varepsilon}(u) \in G_{c 2 \delta}\left(\mathcal{Z}_{\varepsilon}^{10 \beta}\right) \quad \text { for } u \in G_{c 2 \delta}\left(\mathcal{Z}_{\varepsilon}^{10 \beta}\right) .
$$

It is important to note that for any $u \in G_{2 \delta}\left(\mathcal{Z}_{\varepsilon}^{10 \beta}\right)$,

$$
\Gamma_{\varepsilon}\left(\tau_{\varepsilon}(u)\right) \leq \Gamma_{\varepsilon}(u) .
$$

Now we prove the following result. 


\section{Proposition 10.}

$$
\left|\Upsilon_{\varepsilon}\left(\tau_{\varepsilon}(u)\right)-\Upsilon_{\varepsilon}(u)\right|<2 R_{0} \quad \text { for any } u \in G_{2 \delta}\left(\mathcal{Z}_{\varepsilon}^{10 \beta}\right) \text {. }
$$

Proof. By Proposition 3 there exist $U \in S$ and $y \in \Omega^{10 \beta}$ such that $\left|y-\Upsilon_{\varepsilon}(u)\right| \leq R_{0}$ and $\left\|u-\phi_{\varepsilon}(\cdot-y) U(\cdot-y)\right\|_{\varepsilon} \leq 2 \delta$. Then Proposition 9 implies that for small $\varepsilon>0$,

$$
\begin{aligned}
\| \tau_{\varepsilon}(u) & -\phi_{\varepsilon}(\cdot-y) U(\cdot-y) \|_{\varepsilon}^{2} \\
= & \int_{B\left(\Upsilon_{\varepsilon}(u), 1 / \sqrt{\varepsilon}\right)}\left[\left|\nabla\left(u-\phi_{\varepsilon}(\cdot-y) U(\cdot-y)\right)\right|^{2}+V_{\varepsilon}\left(u-\phi_{\varepsilon}(\cdot-y) U(\cdot-y)\right)^{2}\right] d x \\
& +\int_{\mathbb{R}^{N} \backslash B\left(\Upsilon_{\varepsilon}(u), 1 / \sqrt{\varepsilon}\right)}\left[\left|\nabla \tau_{\varepsilon}(u)\right|^{2}+V_{\varepsilon} \tau_{\varepsilon}(u)^{2}\right] d x \\
\leq & (2 \delta)^{2}+(2 \sqrt{2} \delta)^{2} \leq(4 \delta)^{2} .
\end{aligned}
$$

Note that for $\left|z-\Upsilon_{\varepsilon}(u)\right| \geq 2 R_{0}$,

$$
|y-z| \geq\left|z-\Upsilon_{\varepsilon}(u)\right|-\left|y-\Upsilon_{\varepsilon}(u)\right| \geq R_{0} .
$$

Thus, for $\left|z-\Upsilon_{\varepsilon}(u)\right| \geq 2 R_{0}$, it follows from (21) and (22) that

$$
\begin{aligned}
\| \tau_{\varepsilon}(u)- & \phi_{\varepsilon}(\cdot-z) U(\cdot-z) \|_{\varepsilon} \\
& \geq\left\|\phi_{\varepsilon}(\cdot-y) U(\cdot-y)-\phi_{\varepsilon}(\cdot-z) U(\cdot-z)\right\|_{\varepsilon}-\left\|\tau_{\varepsilon}(u)-\phi_{\varepsilon}(\cdot-y) U(\cdot-y)\right\|_{\varepsilon} \\
& \geq \xi / 2-4 \delta \geq 6 \delta .
\end{aligned}
$$

Hence $\sigma\left(\operatorname{dist}_{\varepsilon}\left(\tau_{\varepsilon}(u), S(z)\right)\right)=0$ if $\left|z-\Upsilon_{\varepsilon}(u)\right| \geq 2 R_{0}$. This implies that $\Upsilon_{\varepsilon}\left(\tau_{\varepsilon}(u)\right) \in$ $B\left(\Upsilon_{\varepsilon}(u), 2 R_{0}\right)$.

Note that for $z \in L_{\varepsilon}$ and $t \in[0, T]$,

$$
A_{\varepsilon}(t, z)(x)=\phi_{\varepsilon}\left(x / t-\Theta_{\varepsilon}(z) / t \varepsilon\right) U\left(x / t-\Theta_{\varepsilon}(z) / t \varepsilon\right)
$$

and

$$
\operatorname{supp}(A(t, z)) \subset B\left(\Theta_{\varepsilon}(z) / \varepsilon, 1 / \varepsilon^{1 / 3}\right) .
$$

Now, since $\left\{A_{\varepsilon}(t, z) \mid t \in[0, T], z \in L_{\varepsilon}\right\}$ is compact, we can extend the center of mass $\Upsilon_{\varepsilon}$ on $G_{2 \delta}\left(\mathcal{Z}_{\varepsilon}^{10 \beta}\right)$ to a function $\widetilde{\Upsilon}_{\varepsilon}$ on

$$
G_{2 \delta}\left(\mathcal{Z}_{\varepsilon}^{10 \beta}\right) \cup\left\{A_{\varepsilon}(t, z) \mid t \in[0, T], z \in L_{\varepsilon}\right\}
$$

so that for any $t \in[0, T]$ and $z \in \Omega_{\varepsilon}^{10 \beta}$ with $A_{\varepsilon}(t, z) \notin G_{2 \delta}\left(\mathcal{Z}_{\varepsilon}^{10 \beta}\right)$,

$$
\left|\widetilde{\Upsilon}_{\varepsilon}\left(A_{\varepsilon}(t, z)\right)-\Theta_{\varepsilon}(z) / \varepsilon\right| \leq 3 R_{0}
$$

and

$$
\widetilde{\Upsilon}_{\varepsilon}\left(A_{\varepsilon}(t, z)\right)=\Theta_{\varepsilon}(z) / \varepsilon \quad \text { in a neighborhood } N \text { of } \partial\left([0, T] \times L_{\varepsilon}\right) .
$$

Then we see that if $\left|x-\widetilde{\Upsilon}_{\varepsilon}\left(A_{\varepsilon}(t, z)\right)\right|=1 / \sqrt{\varepsilon}$, then $A_{\varepsilon}(t, z)(x)=0$ for small $\varepsilon>0$. Thus we can also extend $\tau_{\varepsilon}$ continuously onto $G_{2 \delta}\left(\mathcal{Z}_{\varepsilon}^{10 \beta}\right) \cup\left\{A_{\varepsilon}(t, z) \mid t \in[0, T], z \in L_{\varepsilon}\right\}$ so that for $\varepsilon>0$ is sufficiently small,

$$
\tau_{\varepsilon}\left(A_{\varepsilon}(t, z)\right)=A_{\varepsilon}(t, z) \quad \text { if } A_{\varepsilon}(t, z) \notin G_{2 \delta}\left(\mathcal{Z}_{\varepsilon}^{10 \beta}\right) .
$$




\section{Energy decreasing deformation through a flow $\Phi$}

We take a large $D>\max \{1,10 \beta\}$ so that $O \subset B(0, D)$, and define

$$
\omega \equiv \max _{|x| \leq 10 D} V(x) / \min _{|x| \leq 10 D} V(x)>1 .
$$

Then we find $\psi_{\varepsilon} \in C_{0}^{\infty}\left(\mathbb{R}^{N},[0,1]\right)$ such that $\psi_{\varepsilon}(x)=0$ for $|x| \geq 8 D / \varepsilon, \psi_{\varepsilon}(x)=1$ for $|x| \leq 7 D / \varepsilon$ and $\left|\nabla \psi_{\varepsilon}\right| \leq \varepsilon$. We pick $\varphi_{1} \in C_{0}^{\infty}\left(\mathbb{R}^{N},[0,1]\right)$ such that $\varphi_{1}(x)=1$ for $x \in \Omega^{6 \beta} \backslash \Omega^{3 \beta}$, and $\varphi_{1}(x)=0$ for $x \in \Omega^{2 \beta} \cup\left(\mathbb{R}^{N} \backslash \Omega^{7 \beta}\right)$. We also select $\varphi_{2} \in$ $C^{2}\left(G_{2 \delta}\left(\mathcal{Z}_{\varepsilon}^{10 \beta}\right),[0,1]\right)$ such that $\varphi_{2}(u)=1$ for $u \in G_{\delta / 6 \omega}\left(\mathcal{Z}^{10 \beta}\right)$ and $\varphi_{2}(u)=0$ for $u \notin G_{\delta / 4 \omega}\left(\mathcal{Z}^{10 \beta}\right)$. Then we define a function $\Psi:[0, \infty) \times \mathbb{R}^{N} \times H_{\varepsilon} \rightarrow \mathbb{R}^{N}$ by

$$
\Psi(l, x, u) \equiv \Phi\left(\varphi_{1}(x) \varphi_{2}(u) l, x\right) .
$$

We fix $l_{p} \in(0,1)$ so that

$$
|\Phi(l, x)-x| \leq \beta / 10 \quad \text { for any } l \in\left[0, l_{p}\right] \text { and } x \in \Omega^{8 \beta} .
$$

Then we define $P_{\varepsilon}:\left[0, l_{p}\right] \times G_{2 \delta}\left(\mathcal{Z}_{\varepsilon}^{10 \beta}\right) \rightarrow H_{\varepsilon}$ by

$$
P_{\varepsilon}(l, u)(x) \equiv\left(1-\psi_{\varepsilon}(x)\right) u(x)+\left(\psi_{\varepsilon} u\right)\left(x-\frac{\Psi\left(l, \varepsilon \Upsilon_{\varepsilon}(u), u\right)}{\varepsilon}+\Upsilon_{\varepsilon}(u)\right) .
$$

We note that $P_{\varepsilon}(l, u)=u$ for any $l \in\left[0, l_{p}\right]$ if $\Upsilon_{\varepsilon}(u) \in \Omega_{\varepsilon}^{2 \beta} \cup\left(\mathbb{R}^{N} \backslash \Omega_{\varepsilon}^{7 \beta}\right)$ or $u \notin$ $G_{\delta / 4 \omega}\left(\mathcal{Z}_{\varepsilon}^{10 \beta}\right)$. We take a small $\delta>0$ so that $q=q(\delta)$ given in Proposition 5 satisfies $1-q>1 / \sqrt{2}$. Through the deformation $P_{\varepsilon}$, the center of mass $\Upsilon_{\varepsilon}$ moves roughly like the deformation $\Psi$, as we see in the following proposition.

Proposition 11. For $u \in G_{2 \delta}\left(\mathcal{Z}_{\varepsilon}^{10 \beta}\right)$,

$$
\left|\Upsilon_{\varepsilon}\left(P_{\varepsilon}(l, u)\right)-\Psi\left(l, \varepsilon \Upsilon_{\varepsilon}(u), u\right) / \varepsilon\right| \leq 2 R_{0} .
$$

Proof. Since $\Psi\left(l, \varepsilon \Upsilon_{\varepsilon}(u), u\right)=\varepsilon \Upsilon_{\varepsilon}(u)$ and $P_{\varepsilon}(l, u)=u$ if $u \in G_{2 \delta}\left(\mathcal{Z}_{\varepsilon}^{10 \beta}\right) \backslash G_{\delta / 4 \omega}\left(\mathcal{Z}_{\varepsilon}^{10 \beta}\right)$ or $\Upsilon_{\varepsilon}(u) \notin \Omega_{\varepsilon}^{7 \beta}$, we may assume $u \in G_{\delta / 4 \omega}\left(\mathcal{Z}_{\varepsilon}^{10 \beta}\right)$ and $\Upsilon_{\varepsilon}(u) \in \Omega_{\varepsilon}^{7 \beta}$. Then, from Proposition 3, it suffices to show

$$
\left\|P_{\varepsilon}(l, u)-\phi_{\varepsilon}(\cdot-y) U(\cdot-y)\right\|_{\varepsilon} \leq 2 \delta
$$

for some $U \in S$ and $y \in \mathbb{R}^{N}$ with $\left|y-\Psi\left(l, \varepsilon \Upsilon_{\varepsilon}(u), u\right) / \varepsilon\right| \leq R_{0}$. From Proposition 3, we take $U \in S$ and $z \in \mathbb{R}^{N}$ with $\left|z-\Upsilon_{\varepsilon}(u)\right| \leq R_{0}$ such that

$$
\left\|u-\phi_{\varepsilon}(\cdot-z) U(\cdot-z)\right\|_{\varepsilon} \leq \delta / 4 \omega .
$$

Then $\int_{|x| \geq 7 D / \varepsilon}\left(|\nabla u|^{2}+V_{\varepsilon} u^{2}\right) d x \leq \delta^{2} / 16 \omega^{2}$ since $\operatorname{supp}\left(\phi_{\varepsilon} U\right)(\cdot-z) \subset B(0,7 D / \varepsilon)$. 
Denoting $d_{\varepsilon}(l, u) \equiv \Psi\left(l, \varepsilon \Upsilon_{\varepsilon}(u), u\right) / \varepsilon-\Upsilon_{\varepsilon}(u)$, we deduce that for small $\varepsilon>0$,

$$
\begin{aligned}
\| P_{\varepsilon}(l, u)- & \left(\phi_{\varepsilon} U\right)\left(\cdot-z-d_{\varepsilon}(l, u)\right) \|_{\varepsilon} \\
& \leq\left\|\left(1-\psi_{\varepsilon}\right) u\right\|_{\varepsilon}+\left\|\left(\psi_{\varepsilon} u\right)\left(\cdot-d_{\varepsilon}(l, u)\right)-\left(\phi_{\varepsilon} U\right)\left(\cdot-z-d_{\varepsilon}(l, u)\right)\right\|_{\varepsilon} \\
& \leq \delta / 4 \omega+O(\varepsilon)+\sqrt{\omega}\left\|\psi_{\varepsilon} u-\left(\phi_{\varepsilon} U\right)(\cdot-z)\right\|_{\varepsilon} \\
& \leq \delta / 4 \omega+O(\varepsilon)+\sqrt{\omega}\left\|u-\left(\phi_{\varepsilon} U\right)(\cdot-z)\right\|_{\varepsilon}+\sqrt{\omega}\left\|\left(1-\psi_{\varepsilon}\right) u\right\|_{\varepsilon} \\
& \leq \delta / 2+O(\varepsilon)+\delta / 4 \sqrt{\omega} \leq \delta .
\end{aligned}
$$

Since $\left|z+d_{\varepsilon}(l, u)-\Psi\left(l, \varepsilon \Upsilon_{\varepsilon}(u), u\right) / \varepsilon\right|=\left|z-\Upsilon_{\varepsilon}(u)\right| \leq R_{0}$, this proves the claim.

Proposition 12. If $u \in G_{\delta / 4 \omega}\left(\mathcal{Z}_{\varepsilon}^{10 \beta}\right)$ and $\Upsilon_{\varepsilon}(u) \in \Omega_{\varepsilon}^{7 \beta}$, then $P_{\varepsilon}\left(l, \tau_{\varepsilon}(u)\right) \in G_{\delta / 2}\left(\mathcal{Z}_{\varepsilon}^{10 \beta}\right)$ for $l \in\left[0, l_{p}\right]$; thus $P_{\varepsilon}\left(l, \tau_{\varepsilon}(u)\right) \in G_{2 \delta}\left(\mathcal{Z}_{\varepsilon}^{10 \beta}\right)$ for all $l \in\left[0, l_{p}\right]$ and $u \in G_{2 \delta}\left(\mathcal{Z}_{\varepsilon}^{10 \beta}\right)$.

Proof. From Proposition 10, we see that $\left|\varepsilon \Upsilon\left(\tau_{\varepsilon}(u)\right)-\varepsilon \Upsilon_{\varepsilon}(u)\right| \leq 2 \varepsilon R_{0}$ for any $u \in$ $G_{2 \delta}\left(\mathcal{Z}_{\varepsilon}^{10 \beta}\right)$. Thus it follows from (46) that for small $\varepsilon>0$,

$$
\Upsilon_{\varepsilon}\left(P_{\varepsilon}\left(l, \tau_{\varepsilon}(u)\right)\right) \in \Omega_{\varepsilon}^{8 \beta} \quad \text { when } l \in\left[0, l_{p}\right] .
$$

We recall that for $u \in N_{2 \delta}\left(\mathcal{Z}_{\varepsilon}^{10 \beta}\right)$ and $v \in H_{\varepsilon}$,

$$
|v|_{\varepsilon, u} \equiv \int_{\left|x-\Upsilon_{\varepsilon}(u)\right| \leq 1 / \sqrt{\varepsilon}}\left[|\nabla v|^{2}+V_{\varepsilon} v^{2}\right] d x .
$$

Since $u \in G_{\delta / 4 \omega}\left(\mathcal{Z}_{\varepsilon}^{10 \beta}\right)$, it follows that $\tau_{\varepsilon}(u) \in G_{\delta / 4 \omega}\left(\mathcal{Z}_{\varepsilon}^{10 \beta}\right) \subset N_{\delta / 4 \omega(1-q)}\left(\mathcal{Z}_{\varepsilon}^{10 \beta}\right)$ and

$$
\int_{\left|y-\Upsilon_{\varepsilon}\left(\tau_{\varepsilon}(u)\right)\right| \geq 1 / \sqrt{\varepsilon}}\left[\left|\nabla \tau_{\varepsilon}(u)\right|^{2}+V_{\varepsilon}\left(\tau_{\varepsilon}(u)\right)^{2}-2 F\left(\tau_{\varepsilon}(u)\right)\right] d y \leq(\delta / 4 \omega)^{2} / 2 .
$$

Since $\tau_{\varepsilon}(u) \in N_{\delta / 4 \omega(1-q)}\left(\mathcal{Z}_{\varepsilon}^{10 \beta}\right)$ and $\Upsilon_{\varepsilon}\left(\tau_{\varepsilon}(u)\right) \in \Omega_{\varepsilon}^{8 \beta}$ for small $\varepsilon>0$, it follows that for some $z \in \Omega^{9 \beta}$ and $U \in S$,

$$
\left\|\tau_{\varepsilon}(u)-\phi_{\varepsilon}(\cdot-z / \varepsilon) U(\cdot-z / \varepsilon)\right\|_{\varepsilon} \leq \delta / 4 \omega(1-q) .
$$

Then, denoting

$$
d_{\varepsilon}(l, u) \equiv \frac{\Psi\left(l, \varepsilon \Upsilon_{\varepsilon}\left(\tau_{\varepsilon}(u)\right), \tau_{\varepsilon}(u)\right)}{\varepsilon}-\Upsilon_{\varepsilon}\left(\tau_{\varepsilon}(u)\right), \quad g_{\varepsilon}(l, u) \equiv \Upsilon_{\varepsilon}\left(P_{\varepsilon}\left(l, \tau_{\varepsilon}(u)\right)\right),
$$

we see from a change of variables that for small $\varepsilon>0$ and $l \in\left(0, l_{p}\right)$,

$$
\begin{aligned}
& \left|P_{\varepsilon}\left(l, \tau_{\varepsilon}(u)\right)(\cdot)-\phi_{\varepsilon}\left(\cdot-z / \varepsilon-d_{\varepsilon}(l, u)\right) U\left(\cdot-z / \varepsilon-d_{\varepsilon}(l, u)\right)\right|_{\varepsilon, P_{\varepsilon}\left(l, \tau_{\varepsilon}(u)\right)} \\
& =\int_{\left|y-g_{\varepsilon}(l, u)+d_{\varepsilon}(l, u)\right| \leq 1 / \sqrt{\varepsilon}}\left|\nabla\left(\tau_{\varepsilon}(u)(y)\right)-\nabla\left(\phi_{\varepsilon}(y-z / \varepsilon) U(y-z / \varepsilon)\right)\right|^{2} d y \\
& \quad+\int_{\left|y-g_{\varepsilon}(l, u)+d_{\varepsilon}(l, u)\right| \leq 1 / \sqrt{\varepsilon}} V_{\varepsilon}\left(y+d_{\varepsilon}(l, u)\right)\left(\tau_{\varepsilon}(u)(y)-\phi_{\varepsilon}(y-z / \varepsilon) U(y-z / \varepsilon)\right)^{2} d y .
\end{aligned}
$$


Here, we have used the fact that

$$
P_{\varepsilon}\left(l, \tau_{\varepsilon}(u)\right)(y)=\tau_{\varepsilon}(u)\left(y-d_{\varepsilon}(l, u)\right) \quad \text { for }\left|y-g_{\varepsilon}(l, e)+d_{\varepsilon}(l, u)\right| \leq 1 / \sqrt{\varepsilon} .
$$

Note that if $\left|y-g_{\varepsilon}(l, u)+d_{\varepsilon}(l, u)\right| \leq 1 / \sqrt{\varepsilon}$, then $\left|\varepsilon y+\varepsilon d_{\varepsilon}(l, u)\right| \leq 2 D,|\varepsilon y| \leq 3 D$ for small $\varepsilon>0$ since $\varepsilon g_{\varepsilon}(l, u) \in \Omega^{9 \beta} \subset B(0, D)$ for $l \in\left[0, l_{p}\right]$ and small $\varepsilon>0$. It follows from the definition of $\omega$ in (45) that if $\left|y-g_{\varepsilon}(l, u)+d_{\varepsilon}(l, u)\right| \leq 1 / \sqrt{\varepsilon}$,

$$
V\left(\varepsilon y+\varepsilon d_{\varepsilon}(l, u)\right) \leq \omega V(\varepsilon y) .
$$

Consequently,

$$
\begin{aligned}
\mid P_{\varepsilon}\left(l, \tau_{\varepsilon}(u)\right)-\phi_{\varepsilon}(\cdot- & \left.z / \varepsilon-d_{\varepsilon}(l, u)\right)\left.U\left(\cdot-z / \varepsilon-d_{\varepsilon}(l, u)\right)\right|_{\varepsilon, P_{\varepsilon}\left(l, \tau_{\varepsilon}(u)\right)} \\
\leq & \omega\left\|\tau_{\varepsilon}(u)-\phi_{\varepsilon}(\cdot-z / \varepsilon) U(\cdot-z / \varepsilon)\right\|_{\varepsilon}^{2} \\
\leq & \omega(\delta / 4 \omega(1-q))^{2}=\left(\frac{\delta}{2}\right)^{2} \frac{1}{4 \omega(1-q)^{2}}<\frac{1}{2}\left(\frac{\delta}{2}\right)^{2} .
\end{aligned}
$$

We recall that for $l \in\left(0, l_{p}\right)$,

$$
\begin{aligned}
P_{\varepsilon}\left(l, \tau_{\varepsilon}(u)\right)(x)= & \left(1-\psi_{\varepsilon}(x)\right) \tau_{\varepsilon}(u)(x) \\
& +\left(\psi_{\varepsilon} \tau_{\varepsilon}(u)\right)\left(x-\frac{\Psi\left(l, \varepsilon \Upsilon_{\varepsilon}\left(\tau_{\varepsilon}(u)\right), \tau_{\varepsilon}(u)\right)}{\varepsilon}+\Upsilon_{\varepsilon}\left(\tau_{\varepsilon}(u)\right)\right)
\end{aligned}
$$

and

$$
g_{\varepsilon}(l, u)-d_{\varepsilon}(l, u)-\Upsilon_{\varepsilon}\left(\tau_{\varepsilon}(u)\right)=\Upsilon_{\varepsilon}\left(P_{\varepsilon}\left(l, \tau_{\varepsilon}(u)\right)\right)-\frac{\Psi\left(l, \varepsilon \Upsilon_{\varepsilon}\left(\tau_{\varepsilon}(u)\right), \tau_{\varepsilon}(u)\right)}{\varepsilon} .
$$

It is easy to see from Proposition 9 that for some $C, c>0$,

$$
\int_{\mathbb{R}^{N} \backslash B(0,5 D / \varepsilon)}\left[\left|\nabla\left(\left(1-\psi_{\varepsilon}\right) \tau_{\varepsilon}(u)\right)\right|^{2}+V_{\varepsilon}\left(\left(1-\psi_{\varepsilon}\right) \tau_{\varepsilon}(u)\right)^{2}\right] d x \leq C \exp (-c \varepsilon) .
$$

From (48) and Proposition 3, we see that

$$
\left|\Upsilon_{\varepsilon}\left(\tau_{\varepsilon}(u)\right)-z / \varepsilon\right| \leq R_{0} \quad \text { and } \quad\left|\Upsilon_{\varepsilon}\left(\psi_{\varepsilon} \tau_{\varepsilon}(u)\right)-z / \varepsilon\right| \leq R_{0}+o(\varepsilon)
$$

From (46), we deduce that $\left|d_{\varepsilon}\right| \leq \beta / \varepsilon$. Then (45) implies that for small $\varepsilon>0$,

$$
\begin{aligned}
& \left\|\left(\psi_{\varepsilon} \tau_{\varepsilon}(u)\right)\left(\cdot-d_{\varepsilon}(l, u)\right)-\left(\phi_{\varepsilon} U\right)\left(\cdot-z / \varepsilon-d_{\varepsilon}(l, u)\right)\right\|_{\varepsilon}^{2} \\
& \quad \leq \omega\left\|\psi_{\varepsilon} \tau_{\varepsilon}(u)-\left(\phi_{\varepsilon} U\right)(\cdot-z / \varepsilon)\right\|_{\varepsilon}^{2} \leq \omega(\delta / 4 \omega(1-q))^{2}+o(1) .
\end{aligned}
$$

Now, it follows from Proposition 3 that for small $\varepsilon>0$,

$$
\left|\Upsilon_{\varepsilon}\left(\left(\psi_{\varepsilon} \tau_{\varepsilon}(u)\right)\left(\cdot-d_{\varepsilon}(l, u)\right)\right)-z / \varepsilon-d_{\varepsilon}(l, u)\right| \leq R_{0} .
$$


This implies that for $l \in\left[0, l_{p}\right]$,

$$
\begin{aligned}
& \left|g_{\varepsilon}(l, u)-d_{\varepsilon}(l, u)-\Upsilon_{\varepsilon}\left(\tau_{\varepsilon}(u)\right)\right| \\
& \quad \leq\left|g_{\varepsilon}(l, u)-d_{\varepsilon}(l, u)-z / \varepsilon\right|+\left|z / \varepsilon-\Upsilon_{\varepsilon}\left(\tau_{\varepsilon}(u)\right)\right| \leq 2 R_{0}+o(1)
\end{aligned}
$$

when $\varepsilon>0$ is small. It follows from Proposition 9 that for small $\varepsilon>0$,

$$
\int_{\left|x-\Upsilon_{\varepsilon}\left(\tau_{\varepsilon}(u)\right)\right| \geq 1 / \sqrt{\varepsilon}}\left[\left|\nabla \tau_{\varepsilon}(u)\right|^{2}+V_{\varepsilon}(x)\left(\tau_{\varepsilon}(u)\right)^{2}\right] d x \leq(\delta / 4 \omega(1-q))^{2}+o(1) .
$$

Moreover, it follows from (25) that for $l \in\left[0, l_{p}\right]$,

$$
\begin{aligned}
& \int_{\left|x-g_{\varepsilon}(l, u)\right| \geq 1 / \sqrt{\varepsilon}} {\left[\left|\nabla P_{\varepsilon}\left(l, \tau_{\varepsilon}(u)\right)\right|^{2}+V_{\varepsilon}(x)\left(P_{\varepsilon}\left(l, \tau_{\varepsilon}(u)\right)\right)^{2}-2 F\left(P_{\varepsilon}\left(l, \tau_{\varepsilon}(u)\right)\right)\right] d x } \\
& \leq(1+q) \int_{\left|x-g_{\varepsilon}(l, u)\right| \geq 1 / \sqrt{\varepsilon}}\left[\left|\nabla P_{\varepsilon}\left(l, \tau_{\varepsilon}(u)\right)\right|^{2}+V_{\varepsilon}(x)\left(P_{\varepsilon}\left(l, \tau_{\varepsilon}(u)\right)\right)^{2}\right] d x .
\end{aligned}
$$

We see from (50) that for small $\varepsilon>0$ and $l \in\left[0, l_{p}\right]$,

$$
\begin{aligned}
& \int_{\left|x-g_{\varepsilon}(l, u)\right| \geq 1 / \sqrt{\varepsilon}}\left[\left|\nabla P_{\varepsilon}\left(l, \tau_{\varepsilon}(u)\right)\right|^{2}+V_{\varepsilon}(x)\left(P_{\varepsilon}\left(l, \tau_{\varepsilon}(u)\right)\right)^{2}\right] d x \\
& \quad \leq \int_{\left|x-g_{\varepsilon}(l, u)+d_{\varepsilon}(l, u)\right| \geq 1 / \sqrt{\varepsilon}}\left[\left|\nabla\left(\psi_{\varepsilon} \tau_{\varepsilon}(u)\right)\right|^{2}+V_{\varepsilon}\left(x+d_{\varepsilon}(u)\right)\left(\psi_{\varepsilon} \tau_{\varepsilon}(u)\right)^{2}\right] d x+o(\varepsilon) \\
& \quad \leq \omega \int_{\left|x-g_{\varepsilon}(l, u)+d_{\varepsilon}(l, u)\right| \geq 1 / \sqrt{\varepsilon},|x| \leq 10 D / \varepsilon}\left[\left|\nabla \tau_{\varepsilon}(u)\right|^{2}+V_{\varepsilon}(x)\left(\tau_{\varepsilon}(u)\right)^{2}\right] d x+o(\varepsilon) \\
& \leq \omega\left\|\tau_{\varepsilon}(u)-\phi_{\varepsilon}(\cdot-z / \varepsilon) U(\cdot-z / \varepsilon)\right\|_{\varepsilon}^{2}+o(\varepsilon) \leq \omega(\delta / 4 \omega(1-q))^{2}+o(\varepsilon) .
\end{aligned}
$$

Thus, for $l \in\left[0, l_{p}\right]$ and small $\varepsilon>0$,

$$
\begin{aligned}
\int_{\left|x-g_{\varepsilon}(l, u)\right| \geq 1 / \sqrt{\varepsilon}}\left[\left|\nabla P_{\varepsilon}\left(l, \tau_{\varepsilon}(u)\right)\right|^{2}+V_{\varepsilon}(x)\left(P_{\varepsilon}\left(l, \tau_{\varepsilon}(u)\right)\right)^{2}-2 F\left(P_{\varepsilon}\left(l, \tau_{\varepsilon}(u)\right)\right)\right] d x \\
\leq \omega(1+q)(\delta / 4 \omega(1-q))^{2}+o(\varepsilon) .
\end{aligned}
$$

Now, taking sufficiently small $\delta>0$, we see that for small $\varepsilon>0$ and $l \in\left(0, l_{p}\right)$,

$\int_{\left.\mid x-g_{\varepsilon}(l, u)\right) \mid \geq 1 / \sqrt{\varepsilon}}\left[\left|\nabla P_{\varepsilon}\left(l, \tau_{\varepsilon}(u)\right)\right|^{2}+V_{\varepsilon}\left(P_{\varepsilon}\left(l, \tau_{\varepsilon}(u)\right)\right)^{2}-2 F\left(P_{\varepsilon}\left(l, \tau_{\varepsilon}(u)\right)\right)\right] d x \leq \frac{1}{2}\left(\frac{\delta}{2}\right)^{2}$,

and

$$
\left|P_{\varepsilon}\left(l, \tau_{\varepsilon}(u)\right)-\phi_{\varepsilon}\left(\cdot-z / \varepsilon-d_{\varepsilon}(u)\right) U\left(\cdot-z / \varepsilon-d_{\varepsilon}(u)\right)\right|_{\varepsilon, P_{\varepsilon}\left(l, \tau_{\varepsilon}(u)\right)} \leq \frac{1}{2}\left(\frac{\delta}{2}\right)^{2} .
$$

This proves the first claim. 
If $\tau_{\varepsilon}(u) \in G_{\delta / 4 \omega}\left(\Omega^{10 \beta}\right)$ and $\Upsilon_{\varepsilon}\left(\tau_{\varepsilon}(u)\right) \in \Omega_{\varepsilon}^{7 \beta}$ for $u \in G_{2 \delta}\left(\Omega^{10 \beta}\right)$, it follows from the first claim that $P_{\varepsilon}\left(l, \tau_{\varepsilon}(u)\right) \in G_{2 \delta}\left(\Omega^{10 \beta}\right)$ for $l \in\left[0, l_{p}\right]$. If $\tau_{\varepsilon}(u) \notin G_{\delta / 4 \omega}\left(\Omega^{10 \beta}\right)$ and $\Upsilon_{\varepsilon}\left(\tau_{\varepsilon}(u)\right) \in \Omega_{\varepsilon}^{7 \beta}$ for $u \in G_{2 \delta}\left(\Omega^{10 \beta}\right)$, we see from the fact $\operatorname{supp}\left(\varphi_{2}\right) \subset G_{\delta / 4 \omega}\left(\Omega^{10 \beta}\right)$ that $P_{\varepsilon}\left(l, \tau_{\varepsilon}(u)\right)=\tau_{\varepsilon}(u)$. If $\Upsilon_{\varepsilon}\left(\tau_{\varepsilon}(u)\right) \notin \Omega_{\varepsilon}^{7 \beta}$ for some $u \in G_{2 \delta}\left(\Omega^{10 \beta}\right)$ it follows from the facts $\operatorname{supp}\left(\varphi_{1}\right) \subset \Omega^{7 \beta} \backslash \Omega^{2 \beta}$ that $P_{\varepsilon}\left(l, \tau_{\varepsilon}(u)\right)=\tau_{\varepsilon}(u)$. Since $\tau_{\varepsilon}$ is a map from $G_{2 \delta}\left(\Omega^{10 \beta}\right)$ to itself, the second claim follows.

Proposition 13. For $u \in G_{2 \delta}\left(\mathcal{Z}_{\varepsilon}^{10 \beta}\right)$ with $\Upsilon_{\varepsilon}(u) \in \Omega_{\varepsilon}^{8 \beta}$ and small $\varepsilon>0$, the energy functional $\Gamma_{\varepsilon}\left(P_{\varepsilon}\left(l, \tau_{\varepsilon}(u)\right)\right)$ is nonincreasing with respect to $l \in\left[0, l_{p}\right]$. If $u \in$ $G_{\delta / 10 \omega}\left(\mathcal{Z}^{10 \beta}\right)$ and $\Upsilon_{\varepsilon}(u) \in \Omega_{\varepsilon}^{5 \beta} \backslash \Omega_{\varepsilon}^{4 \beta}$, there exists a constant $\mu_{0}>0$, independent of small $\varepsilon>0$, such that

$$
\Gamma_{\varepsilon}\left(P_{\varepsilon}\left(l_{p}, \tau_{\varepsilon}(u)\right)\right)-\Gamma_{\varepsilon}\left(P_{\varepsilon}\left(0, \tau_{\varepsilon}(u)\right)\right) \leq-\mu_{0} .
$$

Proof. We see from a change of variables that for $0 \leq l<l+l^{\prime} \leq l_{p}$,

$$
\begin{aligned}
& \Gamma_{\varepsilon}\left(P_{\varepsilon}\left(l+l^{\prime}, \tau_{\varepsilon}(u)\right)\right)-\Gamma_{\varepsilon}\left(P_{\varepsilon}\left(l, \tau_{\varepsilon}(u)\right)\right) \\
&=\int_{\mathbb{R}^{N}} \nabla\left(\left(\left(1-\psi_{\varepsilon}\right) \tau_{\varepsilon}(u)\right)(x)\right) \cdot \nabla\left(\left(\psi_{\varepsilon} \tau_{\varepsilon}(u)\right)\left(x-d_{\varepsilon}\left(l+l^{\prime}, u\right)\right)\right) d x \\
&-\int_{\mathbb{R}^{N}} \nabla\left(\left(\left(1-\psi_{\varepsilon}\right) \tau_{\varepsilon}(u)\right)(x)\right) \cdot \nabla\left(\left(\psi_{\varepsilon} \tau_{\varepsilon}(u)\right)\left(x-d_{\varepsilon}(l, u)\right)\right) d x \\
&+\int_{\mathbb{R}^{N}} V_{\varepsilon}\left(\left(1-\psi_{\varepsilon}\right) \tau_{\varepsilon}(u)\right)(x)\left(\psi_{\varepsilon} \tau_{\varepsilon}(u)\right)\left(x-d_{\varepsilon}\left(l+l^{\prime}, u\right)\right) d x \\
&-\int_{\mathbb{R}^{N}} V_{\varepsilon}\left(\left(1-\psi_{\varepsilon}\right) \tau_{\varepsilon}(u)\right)(x)\left(\psi_{\varepsilon} \tau_{\varepsilon}(u)\right)\left(x-d_{\varepsilon}(l, u)\right) d x \\
&-\int_{\mathbb{R}^{N}}\left[F \left(P_{\varepsilon}\left(l+l^{\prime}, \tau_{\varepsilon}(u)\right)-F\left(P_{\varepsilon}\left(l, \tau_{\varepsilon}(u)\right)\right] d x\right.\right. \\
&+\frac{1}{2} \int_{\mathbb{R}^{N}} \widetilde{V}_{\varepsilon}\left[\left(\psi_{\varepsilon} \tau_{\varepsilon}(u)\right)\left(x+x_{\varepsilon}\right)\right]^{2} d x \\
& \equiv T I-T I I+T I I I-T I V-T V+T V I,
\end{aligned}
$$

where $x_{\varepsilon} \equiv \Upsilon_{\varepsilon}\left(\tau_{\varepsilon}(u)\right)$ and

$$
\widetilde{V}_{\varepsilon}(x) \equiv V\left(\varepsilon x+\Psi\left(l+l^{\prime}, \varepsilon x_{\varepsilon}, \tau_{\varepsilon}(u)\right)\right)-V\left(\varepsilon x+\Psi\left(l, \varepsilon x_{\varepsilon}, \tau_{\varepsilon}(u)\right)\right) .
$$

If $\varphi_{1}\left(\varepsilon x_{\varepsilon}\right) \varphi_{2}\left(\tau_{\varepsilon}(u)\right)=0$, it follows that $\Psi\left(l, \varepsilon x_{\varepsilon}, u\right)=x_{\varepsilon}$ and thus

$$
P_{\varepsilon}\left(l+l^{\prime}, \tau_{\varepsilon}(u)\right)=P_{\varepsilon}\left(l, \tau_{\varepsilon}(u)\right)=\tau_{\varepsilon}(u) ;
$$

hence the monotonicity property holds. From now on we assume that $\varphi_{1}\left(\varepsilon x_{\varepsilon}\right) \varphi_{2}\left(\tau_{\varepsilon}(u)\right)$ $>0$ and denote

$$
h \equiv \varphi_{1}\left(\varepsilon x_{\varepsilon}\right) \varphi_{2}\left(\tau_{\varepsilon}(u)\right) l^{\prime} .
$$

In this case, $\varepsilon x_{\varepsilon} \in \Omega^{7 \beta} \backslash \Omega^{2 \beta}$. Now, we see that

$$
\frac{1}{h}\left[\Gamma_{\varepsilon}\left(P_{\varepsilon}\left(l+l^{\prime}, \tau_{\varepsilon}(u)\right)\right)-\Gamma_{\varepsilon}\left(P_{\varepsilon}\left(l, \tau_{\varepsilon}(u)\right)\right)\right]=\frac{T I-T I I}{h}+\frac{T I I I-T I V}{h}-\frac{T V}{h}+\frac{T V I}{h} .
$$


From the decay property of $\tau_{\varepsilon}(u)$ in Proposition 9, elliptic estimates and Lipschitz continuity of $\Phi$ in condition (V3), we deduce that for small $\varepsilon>0$,

$$
\left|\frac{T I-T I I}{h}\right|+\left|\frac{T I I I-T I V}{h}\right| \leq o(\varepsilon) .
$$

For an estimate of $T V$, we see from a change of variables and (46) that

$$
\begin{aligned}
T V= & \int_{\mathbb{R}^{N}} F\left(\left(\left(1-\psi_{\varepsilon}\right) \tau_{\varepsilon}(u)\right)\left(x+d_{\varepsilon}\left(l+l^{\prime}, u\right)\right)+\left(\psi_{\varepsilon} \tau_{\varepsilon}(u)\right)(x)\right) d x \\
& -\int_{\mathbb{R}^{N}} F\left(\left(\left(1-\psi_{\varepsilon}\right) \tau_{\varepsilon}(u)\right)\left(x+d_{\varepsilon}(l, u)\right)+\left(\psi_{\varepsilon} \tau_{\varepsilon}(u)\right)(x)\right) d x \\
= & \int_{\mathbb{R}^{N} \backslash B(0,6 D / \varepsilon)} F\left(\left(\left(1-\psi_{\varepsilon}\right) \tau_{\varepsilon}(u)\right)\left(x+d_{\varepsilon}\left(l+l^{\prime}, u\right)\right)+\left(\psi_{\varepsilon} \tau_{\varepsilon}(u)\right)(x)\right) d x \\
& -\int_{\mathbb{R}^{N} \backslash B(0,6 D / \varepsilon)} F\left(\left(\left(1-\psi_{\varepsilon}\right) \tau_{\varepsilon}(u)\right)\left(x+d_{\varepsilon}(l, u)\right)+\left(\psi_{\varepsilon} \tau_{\varepsilon}(u)\right)(x)\right) d x \\
= & \int_{\mathbb{R}^{N} \backslash B(0,6 D / \varepsilon)} \tilde{f}\left(\varepsilon, l, l^{\prime}, x\right) g\left(\varepsilon, l, l^{\prime}, u\right) d x,
\end{aligned}
$$

where

$$
\begin{aligned}
g\left(\varepsilon, l, l^{\prime}, u\right) \equiv & \left(\left(1-\psi_{\varepsilon}\right) \tau_{\varepsilon}(u)\right)\left(x+d_{\varepsilon}\left(l+l^{\prime}, u\right)\right)-\left(\left(1-\psi_{\varepsilon}\right) \tau_{\varepsilon}(u)\right)\left(x+d_{\varepsilon}(l, u)\right), \\
\tilde{f}\left(\varepsilon, l, l^{\prime}, x\right) \equiv & \frac{F\left(\left(\left(1-\psi_{\varepsilon}\right) \tau_{\varepsilon}(u)\right)\left(x+d_{\varepsilon}\left(l+l^{\prime}, u\right)\right)+\left(\psi_{\varepsilon} \tau_{\varepsilon}(u)\right)(x)\right)}{g\left(\varepsilon, l, l^{\prime}, u\right)} \\
& -\frac{F\left(\left(\left(1-\psi_{\varepsilon}\right) \tau_{\varepsilon}(u)\right)\left(x+d_{\varepsilon}(l, u)\right)+\left(\psi_{\varepsilon} \tau_{\varepsilon}(u)\right)(x)\right)}{g\left(\varepsilon, l, l^{\prime}, u\right)} .
\end{aligned}
$$

Then, from the decay property of $\tau_{\varepsilon}(u)$ in Proposition 9, elliptic estimates and Lipschitz continuity of $\Phi$ in condition (V3), we deduce that for small $\varepsilon>0$,

$$
\left|\frac{T V}{h}\right| \leq o(1)
$$

To estimate $T V I / h$, we note that for the constant $c_{2}$ in condition (V3),

$$
\begin{aligned}
\frac{T V I}{h} & =\frac{1}{2} \int_{\mathbb{R}^{N}} \frac{\widetilde{V}_{\varepsilon}}{h}\left[\left(\psi_{\varepsilon} \tau_{\varepsilon}(u)\right)\left(x+x_{\varepsilon}\right)\right]^{2} d x \\
& =\frac{1}{2} \int_{|x| \leq c_{2} / \varepsilon} \frac{\widetilde{V}_{\varepsilon}}{h}\left[\left(\psi_{\varepsilon} \tau_{\varepsilon}(u)\right)\left(x+x_{\varepsilon}\right)\right]^{2} d x+\frac{1}{2} \int_{|x| \geq c_{2} / \varepsilon} \frac{\widetilde{V}_{\varepsilon}}{h}\left[\left(\psi_{\varepsilon} \tau_{\varepsilon}(u)\right)\left(x+x_{\varepsilon}\right)\right]^{2} d x \\
& \equiv A+B .
\end{aligned}
$$


We see that

$$
\begin{aligned}
B= & \frac{1}{2 h} \int_{\left|x-\Psi\left(l+l^{\prime}, \varepsilon x_{\varepsilon}, \tau_{\varepsilon}(u)\right) / \varepsilon\right| \geq \frac{c_{2}}{\varepsilon}} V(\varepsilon x)\left[\left(\psi_{\varepsilon} \tau_{\varepsilon}(u)\right)\left(x-\frac{\Psi\left(l+l^{\prime}, \varepsilon x_{\varepsilon}, \tau_{\varepsilon}(u)\right)}{\varepsilon}+x_{\varepsilon}\right)\right]^{2} d x \\
& -\frac{1}{2 h} \int_{\left|x-\Psi\left(l, \varepsilon x_{\varepsilon}, \tau_{\varepsilon}(u)\right) / \varepsilon\right| \geq \frac{c_{2}}{\varepsilon}} V(\varepsilon x)\left[\left(\psi_{\varepsilon} \tau_{\varepsilon}(u)\right)\left(x-\frac{\Psi\left(l, \varepsilon x_{\varepsilon}, \tau_{\varepsilon}(u)\right)}{\varepsilon}+x_{\varepsilon}\right)\right]^{2} d x .
\end{aligned}
$$

Then, denoting

$$
\begin{aligned}
D_{\varepsilon} \equiv & \left\{x \in \mathbb{R}^{N}|| x-\frac{\Psi_{\varepsilon}\left(l+l^{\prime}, \varepsilon x_{\varepsilon}, \tau_{\varepsilon}(u)\right)}{\varepsilon}\left|\geq \frac{c_{2}}{\varepsilon},\right| x-\frac{\Psi\left(l, \varepsilon x_{\varepsilon}, \tau_{\varepsilon}(u)\right)}{\varepsilon} \mid \leq \frac{c_{2}}{\varepsilon}\right\}, \\
\widetilde{D_{\varepsilon}} \equiv & \left\{x \in \mathbb{R}^{N}|| x-\frac{\Psi_{\varepsilon}\left(l, \varepsilon x_{\varepsilon}, \tau_{\varepsilon}(u)\right)}{\varepsilon}\left|\geq \frac{c_{2}}{\varepsilon},\right| x-\frac{\Psi\left(l+l^{\prime}, \varepsilon x_{\varepsilon}, \tau_{\varepsilon}(u)\right)}{\varepsilon} \mid \leq \frac{c_{2}}{\varepsilon}\right\}, \\
E_{\varepsilon} \equiv & {\left[\left(\psi_{\varepsilon} \tau_{\varepsilon}(u)\right)\left(x-\frac{\Psi_{\varepsilon}\left(l+l^{\prime}, \varepsilon x_{\varepsilon}, \tau_{\varepsilon}(u)\right)}{\varepsilon}+x_{\varepsilon}\right)\right]^{2} } \\
& -\left[\left(\psi_{\varepsilon} \tau_{\varepsilon}(u)\right)\left(x-\frac{\Psi\left(l, \varepsilon x_{\varepsilon}, \tau_{\varepsilon}(u)\right)}{\varepsilon}+x_{\varepsilon}\right)\right]^{2},
\end{aligned}
$$

we see that

$$
\begin{aligned}
|B| \leq & \frac{1}{2 h} \int_{D_{\varepsilon}} V(\varepsilon x)\left[\left(\psi_{\varepsilon} \tau_{\varepsilon}(u)\right)\left(x-\Psi\left(l+l^{\prime}, \varepsilon x_{\varepsilon}, \tau_{\varepsilon}(u)\right) / \varepsilon+x_{\varepsilon}\right)\right]^{2} d x \\
& +\frac{1}{2 h} \int_{\widetilde{D_{\varepsilon}}} V(\varepsilon x)\left[\left(\psi_{\varepsilon} \tau_{\varepsilon}(u)\right)\left(x-\Psi\left(l+l^{\prime}, \varepsilon x_{\varepsilon}, \tau_{\varepsilon}(u)\right) / \varepsilon+x_{\varepsilon}\right)\right]^{2} d x \\
& +\frac{1}{2 h} \int_{\left|x-\Phi\left(l, \varepsilon x_{\varepsilon}, \tau_{\varepsilon}(u)\right) / \varepsilon\right| \geq c_{2} / \varepsilon} V(\varepsilon x)\left|E_{\varepsilon}\right| d x \\
\equiv & B_{1}+B_{2}+B_{3} .
\end{aligned}
$$

It is easy to see that for some constant $M$, depending only on $N$,

$$
\left|D_{\varepsilon}\right|+\left|\widetilde{D_{\varepsilon}}\right| \leq \frac{M}{\varepsilon^{N-1}}\left|\frac{\Psi\left(l+l^{\prime}, \varepsilon x_{\varepsilon}, \tau_{\varepsilon}(u)\right)}{\varepsilon}-\frac{\Psi\left(l, \varepsilon x_{\varepsilon}, \tau_{\varepsilon}(u)\right)}{\varepsilon}\right|
$$

when $l^{\prime}>0$ is small. From the Lipschitz continuity of $\Phi$ in condition (V3), it follows that

$$
\begin{aligned}
& \left|\frac{\Psi\left(l+l^{\prime}, \varepsilon x_{\varepsilon}, \tau_{\varepsilon}(u)\right)}{\varepsilon}-\frac{\Psi\left(l, \varepsilon x_{\varepsilon}, \tau_{\varepsilon}(u)\right)}{\varepsilon}\right| \\
& \quad=\frac{1}{\varepsilon}\left|\Phi\left(\varphi_{1}\left(\varepsilon x_{\varepsilon}\right) \varphi_{2}\left(\tau_{\varepsilon}(u)\right)\left(l+l^{\prime}\right), \varepsilon x_{\varepsilon}\right)-\Phi\left(\varphi_{1}\left(\varepsilon x_{\varepsilon}\right) \varphi_{2}\left(\tau_{\varepsilon}(u)\right) l, \varepsilon x_{\varepsilon}\right)\right| \leq \frac{\mu h}{\varepsilon} .
\end{aligned}
$$

Thus, we see that

$$
\left|D_{\varepsilon}\right|+\left|\widetilde{D_{\varepsilon}}\right| \leq M \mu h / \varepsilon^{N}
$$


For small $h>l^{\prime}>0$, we see from (51) that for $x \in \widetilde{D}_{\varepsilon}$,

$$
\begin{aligned}
& \left|\frac{\Psi\left(l+l^{\prime}, \varepsilon x_{\varepsilon}, \tau_{\varepsilon}(u)\right)}{\varepsilon}-x\right| \\
& \quad \geq\left|x-\frac{\Psi\left(l, \varepsilon x_{\varepsilon}, \tau_{\varepsilon}(u)\right)}{\varepsilon}\right|-\left|\frac{\Psi\left(l+l^{\prime}, \varepsilon x_{\varepsilon}, \tau_{\varepsilon}(u)\right)}{\varepsilon}-\frac{\Psi\left(l, \varepsilon x_{\varepsilon}, \tau_{\varepsilon}(u)\right)}{\varepsilon}\right| \\
& \quad \geq \frac{c_{2}}{\varepsilon}-\frac{\mu h}{\varepsilon} \geq \frac{c_{2}}{2 \varepsilon} .
\end{aligned}
$$

This implies that if $l^{\prime}>0$ is small,

$$
\left|x-\frac{\Psi\left(l, \varepsilon x_{\varepsilon}, \tau_{\varepsilon}(u)\right)}{\varepsilon}\right| \geq \frac{c_{2}}{2 \varepsilon}
$$

for any $x \in D_{\varepsilon} \cup \widetilde{D}_{\varepsilon}$. The decay property of $\tau_{\varepsilon}(u)$ implies that for some $C, c>0$,

$$
\left|B_{1}\right|+\left|B_{2}\right| \leq C \exp (-c / \sqrt{\varepsilon}) .
$$

Denoting $\tilde{x} \equiv x-\Psi\left(l, \varepsilon x_{\varepsilon}, \tau_{\varepsilon}(u)\right) / \varepsilon+x_{\varepsilon}$, we see from the decay property of $\tau_{\varepsilon}(u)=$ $v\left(u, \Upsilon_{\varepsilon}(u), 1 / \sqrt{\varepsilon}\right)$ in Proposition 9, standard $C^{2}$-estimates [22] for the solution of (36) and the Lipschitz continuity of $\Phi$ in condition (V3) that for some $C, c>0$,

$$
\begin{aligned}
\limsup _{l^{\prime} \rightarrow 0}\left|B_{3}\right| & \leq \frac{\mu}{\varepsilon} \int_{\left|x-\Psi\left(l, \varepsilon x_{\varepsilon}, \tau_{\varepsilon}(u)\right) / \varepsilon\right| \geq c_{2} / \varepsilon} V(\varepsilon x)\left(\psi_{\varepsilon} \tau_{\varepsilon}(u)\right)(\tilde{x})\left|\nabla\left(\psi_{\varepsilon} \tau_{\varepsilon}(u)\right)(\tilde{x})\right| d x \\
& \leq C \exp (-c / \sqrt{\varepsilon}) .
\end{aligned}
$$

To estimate $A=\frac{1}{2} \int_{|x| \leq c_{2} / \varepsilon} \frac{\widetilde{V}_{\varepsilon}}{h}\left[\left(\psi_{\varepsilon} \tau_{\varepsilon}(u)\right)\left(x+x_{\varepsilon}\right)\right]^{2} d x$, we denote

$$
H \equiv \varphi_{1}\left(\varepsilon x_{\varepsilon}\right) \varphi_{2}\left(\tau_{\varepsilon}(u)\right) l .
$$

Then we see from condition (V3) that if $\varepsilon x_{\varepsilon} \in \Omega^{7 \beta} \backslash \Omega^{2 \beta}$, then

$$
\begin{aligned}
\limsup _{h \rightarrow 0} \frac{\widetilde{V}_{\varepsilon}}{h} & =\limsup _{h \rightarrow 0} \frac{V\left(\varepsilon x+\Psi\left(l+l^{\prime}, \varepsilon x_{\varepsilon}, \tau_{\varepsilon}(u)\right)\right)-V\left(\varepsilon x+\Psi\left(l, \varepsilon x_{\varepsilon}, \tau_{\varepsilon}(u)\right)\right)}{h} \\
& =\limsup _{h \rightarrow 0} \frac{V\left(\varepsilon x+\Phi\left(H+h, \varepsilon x_{\varepsilon}\right)\right)-V\left(\varepsilon x+\Phi\left(H, \varepsilon x_{\varepsilon}\right)\right)}{h} \leq-\alpha
\end{aligned}
$$

uniformly for $|x| \leq c_{2} / \varepsilon$. Thus, for small $\varepsilon>0$,

$$
\limsup _{l^{\prime} \rightarrow 0} A \leq-\alpha \zeta_{\varepsilon} / 4
$$

where

$$
\zeta_{\varepsilon} \equiv \inf _{u \in G_{2 \delta}\left(\mathcal{Z}_{\varepsilon}^{10 \beta}\right)} \int_{\left|x_{\varepsilon}-x\right| \leq c_{2} / \varepsilon} u^{2} d x>0
$$


Since $\left|x_{\varepsilon}-\Upsilon_{\varepsilon}(u)\right|=\left|\Upsilon_{\varepsilon}\left(\tau_{\varepsilon}(u)\right)-\Upsilon_{\varepsilon}(u)\right| \leq 2 R_{0}$, taking a small $\delta>0$, we see that $\zeta_{0} \equiv \liminf _{\varepsilon \rightarrow 0} \zeta_{\varepsilon}>0$. Thus, combining the estimates for TI, TII, TIII, TIV, TV and $T V I$, we conclude that for sufficiently small $l^{\prime}, \varepsilon>0$,

$$
\frac{1}{h}\left[\Gamma_{\varepsilon}\left(P_{\varepsilon}\left(l+l^{\prime}, \tau_{\varepsilon}(u)\right)\right)-\Gamma_{\varepsilon}\left(P_{\varepsilon}\left(l, \tau_{\varepsilon}(u)\right)\right)\right] \leq-\alpha \zeta_{0} / 8
$$

This proves that $\Gamma_{\varepsilon}\left(P_{\varepsilon}\left(l, \tau_{\varepsilon}(u)\right)\right)$ is decreasing with respect to $l \in\left[0, l_{p}\right]$.

If $u \in G_{\delta / 10 \omega}\left(\mathcal{Z}^{10 \beta}\right)$ and $\Upsilon_{\varepsilon}(u) \in \Omega_{\varepsilon}^{5 \beta} \backslash \Omega_{\varepsilon}^{4 \beta}$, we see from Proposition 10 that $\tau_{\varepsilon}(u) \in G_{\delta / 10 \omega}\left(\mathcal{Z}^{10 \beta}\right), \Upsilon_{\varepsilon}\left(\tau_{\varepsilon}(u)\right) \in \Omega_{\varepsilon}^{6 \beta} \backslash \Omega_{\varepsilon}^{3 \beta}$ for small $\varepsilon>0$. Then $h=$ $\varphi_{1}\left(\varepsilon \Upsilon_{\varepsilon}\left(\tau_{\varepsilon}(u)\right) \varphi_{2}\left(\tau_{\varepsilon}(u)\right) l^{\prime}=l^{\prime}\right.$. Thus, the last claim follows.

\section{Proof of the main theorem}

We will show that for any small $\nu, \delta>0$, there exists a critical point of $\Gamma_{\varepsilon}$ in

$$
\Omega(\varepsilon, v, \delta) \equiv\left(\Gamma_{\varepsilon}^{C_{\varepsilon}} \backslash \Gamma_{\varepsilon}^{E_{m}-v}\right) \cap G_{2 \delta}\left(\mathcal{Z}_{\varepsilon}^{10 \beta}\right)
$$

when $\varepsilon>0$ is sufficiently small. We take a small $\delta>0$ so that $q=q(\delta)<1 / 1000$. Then we see from (26) that

$$
G_{\delta / 3}\left(\mathcal{Z}_{\varepsilon}^{10 \beta}\right) \subset N_{\delta / 2}\left(\mathcal{Z}_{\varepsilon}^{10 \beta}\right) \subset G_{4 \delta / 5}\left(\mathcal{Z}_{\varepsilon}^{10 \beta}\right) \subset N_{\delta}\left(\mathcal{Z}_{\varepsilon}^{10 \beta}\right) .
$$

To the contrary, we assume that for some small $\varepsilon>0$, there exist no critical points of $\Gamma_{\varepsilon}$ in $\Omega(\varepsilon, v, \delta)$. We note that $A_{\varepsilon}(1, z) \in \mathcal{Z}_{\varepsilon}^{10 \beta}$. From Proposition 7 , we can take $v, \delta>0$ so that for small $\varepsilon>0$,

$$
\Gamma_{\varepsilon}\left(A_{\varepsilon}(t, z)\right) \leq E_{m}-v \quad \text { if } \quad A_{\varepsilon}(t, z) \notin G_{\delta / 40 \omega}\left(\mathcal{Z}_{\varepsilon}^{10 \beta}\right) \text { or } \Upsilon_{\varepsilon}\left(A_{\varepsilon}(t, z)\right) \notin \Omega_{\varepsilon}^{2 \beta} .
$$

We take a smooth function $\chi^{\nu}$ on $\mathbb{R}$ such that $\chi^{v}(l)=1$ when $\left|l-E_{m}\right| \leq v / 2$ and $\chi^{v}(l)=0$ for $\left|l-E_{m}\right| \geq v$. We also find a smooth function $\kappa_{\varepsilon}^{\delta}$ on $H_{\varepsilon}$ such that $\kappa_{\varepsilon}^{\delta}(u)=1$ for $u \in G_{\delta}\left(\mathcal{Z}_{\varepsilon}^{10 \beta}\right)$ and $\kappa_{\varepsilon}^{\delta}(u)=0$ for $u \notin G_{2 \delta}\left(\mathcal{Z}_{\varepsilon}^{10 \beta}\right)$. Then we consider the following flow equation:

$$
\frac{d \eta_{\varepsilon}(s, u)}{d s}=-\chi^{v}\left(\Gamma_{\varepsilon}\left(\eta_{\varepsilon}\right)\right) \kappa_{\varepsilon}^{\delta}\left(\eta_{\varepsilon}\right) \Gamma_{\varepsilon}^{\prime}\left(\eta_{\varepsilon}\right) /\left\|\Gamma_{\varepsilon}^{\prime}\left(\eta_{\varepsilon}\right)\right\|_{\varepsilon}^{*}, \quad \eta_{\varepsilon}(0)=u \in H_{\varepsilon} .
$$

There is a unique solution $\eta_{\varepsilon}=\eta_{\varepsilon}(s, u)$ for $s \in[0, \infty)$. Note that if $u \in\left(\Gamma_{\varepsilon}^{C_{\varepsilon}} \backslash \Gamma_{\varepsilon}^{E_{m}-v}\right) \cap$ $G_{2 \delta}\left(\mathcal{Z}_{\varepsilon}^{10 \beta}\right)$, then

$$
\eta_{\varepsilon}(s, u) \in\left(\Gamma_{\varepsilon}^{C_{\varepsilon}} \backslash \Gamma_{\varepsilon}^{E_{m}-v}\right) \cap G_{2 \delta}\left(\mathcal{Z}_{\varepsilon}^{10 \beta}\right) \quad \text { for all } s \in[0, \infty) .
$$

Proposition 14. Let $u \in G_{2 \delta}\left(\mathcal{Z}_{\varepsilon}^{10 \beta}\right)$ and $\Upsilon_{\varepsilon}(u) \in \mathcal{Z}_{\varepsilon}^{9 \beta}$. Suppose that for $0 \leq s_{1}=$ $s_{1}(\varepsilon)<s_{2}=s_{2}(\varepsilon)$ and some constant $c>0$, independent of small $\varepsilon>0$,

$$
\left|\Upsilon_{\varepsilon}\left(\eta_{\varepsilon}\left(s_{1}, u\right)\right)-\Upsilon_{\varepsilon}\left(\eta_{\varepsilon}\left(s_{2}, u\right)\right)\right| \geq c / \varepsilon
$$

Then

$$
\lim _{\varepsilon \rightarrow 0}\left|s_{2}(\varepsilon)-s_{1}(\varepsilon)\right|=\infty
$$


Proof. We consider the function $\Upsilon_{\varepsilon}\left(\eta_{\varepsilon}(s, u)\right), s \in\left[s_{1}, s_{2}\right]$. We can take a partition $s_{1}=$ $s^{0}<s^{1}<\cdots<s^{k-1}<s^{k}=s_{2}$ of $\left[s_{1}, s_{2}\right]$ such that $\left|\Upsilon_{\varepsilon}\left(\eta_{\varepsilon}\left(s^{i}, u\right)\right)-\Upsilon_{\varepsilon}\left(\eta_{\varepsilon}\left(s^{i-1}, u\right)\right)\right| \geq$ $c / k \varepsilon$ for $i=1, \ldots, k$. From Proposition 3 , for each $i=0, \ldots, k$, there exist $z_{i} \in \Omega_{\varepsilon}^{10 \beta}$ and $W_{i} \in S\left(z_{i}\right)$ satisfying

$$
\left\|\eta_{\varepsilon}\left(s^{i}, u\right)-W_{i}\right\|_{\varepsilon} \leq 2 \delta, \quad\left|\Upsilon_{\varepsilon}\left(\eta_{\varepsilon}\left(s^{i}, u\right)\right)-z_{i}\right| \leq R_{0}
$$

Then, for small $\varepsilon>0$, it follows from (21) and (22) that

$$
\begin{aligned}
\| \eta_{\varepsilon}\left(s^{i+1}, u\right)- & \eta_{\varepsilon}\left(s^{i}, u\right) \|_{\varepsilon} \\
& \geq\left\|W_{i}-W_{i+1}\right\|_{\varepsilon}-\left\|\eta_{\varepsilon}\left(s^{i}, u\right)-W_{i}\right\|_{\varepsilon}-\left\|\eta_{\varepsilon}\left(s^{i+1}, u\right)-W_{i+1}\right\|_{\varepsilon} \\
& \geq \xi / 2-4 \delta \geq 10 \delta-4 \delta=6 \delta .
\end{aligned}
$$

Note that $\left\|\partial \eta_{\varepsilon}(s, u) / \partial s\right\|_{\varepsilon}^{*} \leq 1$. Then, it is standard to see that for some $A>0$, independent of $i=1, \ldots, k$ and small $\varepsilon>0,\left|s^{i}-s^{i-1}\right| \geq A$. This implies that for any $k \geq 1$, $\left|s_{1}-s_{2}\right| \geq k A$ if $\varepsilon>0$ is sufficiently small. This proves the claim.

We take a sufficiently small $\delta>0$ so that for $q=q(\delta)$,

$$
\frac{1}{\sqrt{2}(1+q)}-\frac{1}{2(1-q)}>\frac{1}{8}, \quad \frac{1}{10 \sqrt{2}(1+q) \omega}-\frac{1}{30(1-q) \omega}>\frac{1}{60 \omega} .
$$

We remark that since

$$
\frac{1}{\sqrt{2}}-\frac{1}{2}>\frac{1}{8} \text { and } \quad \frac{1}{10 \sqrt{2}}-\frac{1}{30}>\frac{1}{60}
$$

and $\lim _{\delta \rightarrow 0} q(\delta)=0$, there exists a small $\delta$ so that (53) holds.

Then, for $v>0$ defined in (52), we may assume

$$
v<\frac{\delta}{60 \omega} \mu\left(\delta, \frac{\delta}{30 \omega}\right)
$$

where $\mu\left(\delta, \delta^{\prime}\right)$ is defined in Proposition 8 .

Proposition 15. Suppose that $\eta_{\varepsilon}\left(l, u_{\varepsilon}\right) \in G_{\delta / 2}\left(\mathcal{Z}_{\varepsilon}^{10 \beta}\right) \backslash G_{\delta / 10 \omega}\left(\mathcal{Z}_{\varepsilon}^{10 \beta}\right)$ and $\Upsilon_{\varepsilon}\left(\eta_{\varepsilon}\left(l, u_{\varepsilon}\right)\right)$ $\in \Omega_{\varepsilon}^{8 \beta}$ for some $l \in[0, \delta / 30 \omega]$ and $u_{\varepsilon} \in G_{2 \delta}\left(\mathcal{Z}_{\varepsilon}^{10 \beta}\right)$. If $\lim \sup _{\varepsilon \rightarrow 0} \Gamma_{\varepsilon}\left(u_{\varepsilon}\right) \leq E_{m}$, then

$$
\Gamma_{\varepsilon}\left(\eta_{\varepsilon}\left(\frac{\delta}{30 \omega}, u\right)\right) \leq E_{m}-v / 2
$$

for sufficiently small $\varepsilon>0$.

Proof. We note from Proposition 6 that for $c \leq d$,

$$
G_{(1-q) c \delta}\left(\mathcal{Z}_{\varepsilon}^{10 \beta}\right) \subset N_{c \delta}\left(\mathcal{Z}_{\varepsilon}^{10 \beta}\right) \subset N_{d \delta}\left(\mathcal{Z}_{\varepsilon}^{10 \beta}\right) \subset G_{\sqrt{2}(1+q) d \delta}\left(\mathcal{Z}_{\varepsilon}^{10 \beta}\right)
$$


In particular,

$$
\begin{gathered}
G_{\delta / 2}\left(\mathcal{Z}_{\varepsilon}^{10 \beta}\right) \backslash G_{\delta / 10 \omega}\left(\mathcal{Z}_{\varepsilon}^{10 \beta}\right) \subset N_{\delta / 2(1-q)}\left(\mathcal{Z}_{\varepsilon}^{10 \beta}\right) \backslash N_{\delta / 10 \sqrt{2}(1+q) \omega}\left(\mathcal{Z}_{\varepsilon}^{10 \beta}\right), \\
N_{\delta / \sqrt{2}(1+q)}\left(\mathcal{Z}_{\varepsilon}^{10 \beta}\right) \backslash N_{\delta / 30(1-q) \omega}\left(\mathcal{Z}_{\varepsilon}^{10 \beta}\right) \subset G_{\delta}\left(\mathcal{Z}_{\varepsilon}^{10 \beta}\right) \backslash G_{\delta / 30 \omega}\left(\mathcal{Z}_{\varepsilon}^{10 \beta}\right) .
\end{gathered}
$$

First, we suppose that for some $l \in[\delta / 60 \omega, \delta / 30 \omega]$ and $u_{\varepsilon} \in G_{2 \delta}\left(\mathcal{Z}_{\varepsilon}^{10 \beta}\right), \eta_{\varepsilon}\left(l, u_{\varepsilon}\right) \in$ $G_{\delta / 2}\left(\mathcal{Z}_{\varepsilon}^{10 \beta}\right) \backslash G_{\delta / 10 \omega}\left(\mathcal{Z}_{\varepsilon}^{10 \beta}\right)$ and $\Upsilon_{\varepsilon}\left(\eta_{\varepsilon}\left(l, u_{\varepsilon}\right)\right) \in \Omega_{\varepsilon}^{8 \beta}$. Then, since $\left\|d \eta_{\varepsilon} / d s\right\|_{\varepsilon} \leq 1$, we see from Proposition 14 that $\eta_{\varepsilon}\left(s, u_{\varepsilon}\right) \in G_{\delta}\left(\mathcal{Z}_{\varepsilon}^{10 \beta}\right) \backslash G_{\delta / 30 \omega}\left(\mathcal{Z}_{\varepsilon}^{10 \beta}\right)$ and $\Upsilon_{\varepsilon}\left(\eta_{\varepsilon}\left(l, u_{\varepsilon}\right)\right) \in$ $\mathcal{Z}_{\varepsilon}^{9 \beta}$ for $s \in[l-\delta / 60 \omega, l]$ and small $\varepsilon>0$. Then, if $\Gamma_{\varepsilon}\left(\eta_{\varepsilon}\left(s, u_{\varepsilon}\right)\right) \geq E_{m}-v / 2$ for any $s \in[l-\delta / 60 \omega, l]$, it follows that for small $\varepsilon>0, d \eta_{\varepsilon} / d t=-\Gamma_{\varepsilon}^{\prime}\left(\eta_{\varepsilon}\right) /\left\|\Gamma_{\varepsilon}^{\prime}\left(\eta_{\varepsilon}\right)\right\|_{\varepsilon}^{*}$. Note that $\Gamma_{\varepsilon}$ is decreasing along the flow $\eta_{\varepsilon}$. Hence for small $\varepsilon>0$,

$$
\begin{aligned}
\Gamma_{\varepsilon}\left(\eta_{\varepsilon}(l, u)\right)-E_{m}-o(1) & \leq \Gamma_{\varepsilon}\left(\eta_{\varepsilon}\left(l, u_{\varepsilon}\right)\right)-\Gamma_{\varepsilon}\left(\eta_{\varepsilon}\left(l-\delta / 60 \omega, u_{\varepsilon}\right)\right) \\
& =\int_{l-\delta / 60 \omega}^{l} \Gamma_{\varepsilon}^{\prime}\left(\eta_{\varepsilon}\left(s, u_{\varepsilon}\right)\right) \frac{d \eta_{\varepsilon}\left(s, u_{\varepsilon}\right)}{d s} d s=-\int_{l-\delta / 60 \omega}^{l}\left\|\Gamma_{\varepsilon}^{\prime}\right\|_{\varepsilon}^{*} d s \\
& \leq-\frac{\delta}{60 \omega} \mu\left(\delta, \frac{\delta}{30 \omega}\right) \leq-v .
\end{aligned}
$$

This contradicts the assumption that $\Gamma_{\varepsilon}\left(\eta_{\varepsilon}\left(s, u_{\varepsilon}\right)\right) \geq E_{m}-v / 2$ for any $s \in[l-\delta / 60 \omega, l]$. Since $\Gamma_{\varepsilon}$ is decreasing along the flow $\eta_{\varepsilon}$, we conclude that for small $\varepsilon>0$, $\Gamma_{\varepsilon}\left(\eta_{\varepsilon}\left(\delta / 30 \omega, u_{\varepsilon}\right)\right) \leq E_{m}-v / 2$.

Second, we suppose that for some $l \in[0, \delta / 60 \omega]$ and $u_{\varepsilon} \in G_{2 \delta}\left(\mathcal{Z}_{\varepsilon}^{10 \beta}\right), \eta_{\varepsilon}\left(l, u_{\varepsilon}\right) \in$ $G_{\delta / 2}\left(\mathcal{Z}_{\varepsilon}^{10 \beta}\right) \backslash G_{\delta / 10 \omega}\left(\mathcal{Z}_{\varepsilon}^{10 \beta}\right)$ and $\Upsilon_{\varepsilon}\left(\eta_{\varepsilon}\left(l, u_{\varepsilon}\right)\right) \in \Omega_{\varepsilon}^{8 \beta}$. If $\Gamma_{\varepsilon}\left(\eta_{\varepsilon}\left(s, u_{\varepsilon}\right)\right) \geq E_{m}-v / 2$ for any $s \in[l, l+\delta / 60 \omega]$, we get a contradiction by the same procedure as in the first case. Since $l+\delta / 60 \omega \leq \delta / 30 \omega$, we conclude again that for small $\varepsilon>0, \Gamma_{\varepsilon}\left(\eta_{\varepsilon}\left(\delta / 30 \omega, u_{\varepsilon}\right)\right) \leq$ $E_{m}-v / 2$. This completes the proof.

\subsection{Iteration through a gradient flow and a translation}

We define

$$
l_{g} \equiv \frac{\delta}{30 \omega}, \quad I(u) \equiv P_{\varepsilon}\left(l_{p}, \cdot\right) \circ \tau_{\varepsilon} \circ \eta_{\varepsilon}\left(l_{g}, u\right)
$$

and $I^{i}$ the $i$-fold composition of $I$. As before, we denote

$$
\Omega(\varepsilon, v, \delta) \equiv\left(\Gamma_{\varepsilon}^{C_{\varepsilon}} \backslash \Gamma_{\varepsilon}^{E_{m}-v}\right) \cap G_{2 \delta}\left(\mathcal{Z}_{\varepsilon}^{10 \beta}\right) .
$$

Since we assume that there exist no solutions of (13) in $\Omega(\varepsilon, v, \delta)$ for small $\varepsilon>0$, there exists $k(\varepsilon)>0$ such that

$$
\left|\Gamma_{\varepsilon}^{\prime}(u)\right| \geq k(\varepsilon) \quad \text { for any } u \in \Omega(\varepsilon, \nu, \delta) .
$$

We take an integer

$$
j_{\varepsilon} \geq \frac{v}{k(\varepsilon) l_{g}} .
$$

Now, we prove the following proposition. 


\section{Proposition 16.}

$$
\Gamma_{\varepsilon}\left(I^{j_{\varepsilon}}\left(A_{\varepsilon}(t, z)\right)\right) \leq E_{m}-\min \left\{v / 2, \mu_{0} / 2\right\} \quad \text { for any } t \in[0, T], z \in L_{\varepsilon},
$$

where $\mu_{0}>0$ is given in Proposition 13.

Proof. By (52), we recall that if $\Gamma_{\varepsilon}(A(t, z)) \geq E_{m}-v$, then $A(t, z) \in G_{\delta / 40 \omega}\left(\mathcal{Z}_{\varepsilon}^{10 \beta}\right)$ and $\Upsilon_{\varepsilon}(A(t, z)) \in \Omega_{\varepsilon}^{2 \beta}$. Thus, from now on in this proof, it suffices to consider just the cases $A(t, z) \in G_{\delta / 40 \omega}\left(\mathcal{Z}_{\varepsilon}^{10 \beta}\right)$ and $\Upsilon_{\varepsilon}(A(t, z)) \in \Omega_{\varepsilon}^{2 \beta}$. Hence, we consider a sequence of continuous paths

$$
\left\{\eta_{\varepsilon}\left(l, I^{i}\left(A_{\varepsilon}(t, z)\right)\right) \mid l \in\left[0, l_{g}\right]\right\}_{i=0,1, \ldots, j_{\varepsilon}-1} .
$$

Note that $\Gamma_{\varepsilon}$ is nonincreasing along the processes $\eta_{\varepsilon}, \tau_{\varepsilon}, P_{\varepsilon}$. Further we recall the following:

(i) The definition of $\tau_{\varepsilon}$ implies

$$
\tau_{\varepsilon}\left(G_{c \delta}\left(\mathcal{Z}_{\varepsilon}^{10 \beta}\right)\right) \subset G_{c \delta}\left(\mathcal{Z}_{\varepsilon}^{10 \beta}\right) \quad \text { for each } c \in(0,2] .
$$

(ii) Proposition 10 implies

$$
\left|\Upsilon_{\varepsilon}\left(\tau_{\varepsilon}(u)\right)-\Upsilon_{\varepsilon}(u)\right| \leq 2 R_{0} \quad \text { for } u \in G_{2 \delta}\left(\mathcal{Z}_{\varepsilon}^{10 \beta}\right) .
$$

(iii) Proposition 11 implies

$$
\left|\Upsilon_{\varepsilon}\left(P_{\varepsilon}(l, u)\right)-\Psi\left(l, \varepsilon \Upsilon_{\varepsilon}(u), u\right) / \varepsilon\right| \leq 2 R_{0} .
$$

(iv) Proposition 14 implies

$$
\left|\Upsilon_{\varepsilon}\left(\eta_{\varepsilon}\left(l_{g}, u\right)\right)-\Upsilon_{\varepsilon}(u)\right| \leq o(1) / \varepsilon \quad \text { as } \varepsilon \rightarrow 0 ;
$$

thus for small $\varepsilon>0$,

$$
\left\{\Upsilon_{\varepsilon}\left(\eta_{\varepsilon}(l, u)\right) \mid l \in[0, \delta / 30 \omega]\right\} \subset \Omega_{\varepsilon}^{(d+1 / 10) \beta} \quad \text { if } \Upsilon_{\varepsilon}(u) \in \Omega_{\varepsilon}^{d \beta}, d \in[1,9] .
$$

(v) Proposition 12 implies

$$
P_{\varepsilon}\left(l, \tau_{\varepsilon}(u)\right) \in G_{2 \delta}\left(\mathcal{Z}_{\varepsilon}^{10 \beta}\right) \quad \text { for all } l \in\left[0, l_{p}\right] \text { and } u \in G_{2 \delta}\left(\mathcal{Z}_{\varepsilon}^{10 \beta}\right) .
$$

(vi) From the definition of the gradient flow $\eta_{\varepsilon}$,

$$
\left\{\eta_{\varepsilon}(s, u) \mid s \in[0, \infty)\right\} \subset G_{2 \delta}\left(\mathcal{Z}_{\varepsilon}^{10 \beta}\right) \quad \text { for } u \in G_{2 \delta}\left(\mathcal{Z}_{\varepsilon}^{10 \beta}\right) .
$$

Hence, for small $\varepsilon>0$ and each $i=0,1, \ldots, j_{\varepsilon}-1$,

$$
\left|\Upsilon_{\varepsilon}\left(\eta_{\varepsilon}\left(l_{g}, I^{i}\left(A_{\varepsilon}(t, z)\right)\right)\right)-\Upsilon_{\varepsilon}\left(I^{i+1}\left(A_{\varepsilon}(t, z)\right)\right)\right| \leq 4 R_{0}+\beta / 10 \varepsilon
$$

and

$$
\left|\Upsilon_{\varepsilon}\left(\eta_{\varepsilon}\left(l_{g}, I^{i}\left(A_{\varepsilon}(t, z)\right)\right)\right)-\Upsilon_{\varepsilon}\left(I^{i}\left(A_{\varepsilon}(t, z)\right)\right)\right| \leq \beta / 10 \varepsilon
$$


Thus, we deduce that at least one of the following three cases holds:

Case A:

$$
\eta_{\varepsilon}\left(l, I^{i}\left(A_{\varepsilon}(t, z)\right)\right) \in G_{\delta / 10 \omega}\left(\mathcal{Z}_{\varepsilon}^{10 \beta}\right) \cap\left\{u \mid \Upsilon_{\varepsilon}(u) \in \Omega_{\varepsilon}^{5 \beta}\right\}
$$

for all $i=0,1, \ldots, j_{\varepsilon}-1, l \in\left[0, l_{g}\right]$.

\section{Case B:}

$\left\{\eta_{\varepsilon}\left(l, I^{i}\left(A_{\varepsilon}(t, z)\right)\right) \mid l \in\left[0, l_{g}\right]\right\} \subset G_{\delta / 10 \omega}\left(\mathcal{Z}_{\varepsilon}^{10 \beta}\right) \cap\left\{u \mid \Upsilon_{\varepsilon}(u) \in \Omega_{\varepsilon}^{5 \beta-\beta / 10} \backslash \Omega_{\varepsilon}^{4 \beta+\beta / 10}\right\}$ for some $i=0,1, \ldots, j_{\varepsilon}-1$.

Case C:

$$
\eta_{\varepsilon}\left(l, I^{i}\left(A_{\varepsilon}(t, z)\right)\right) \in\left(G_{\delta / 2}\left(\mathcal{Z}_{\varepsilon}^{10 \beta}\right) \backslash G_{\delta / 10 \omega}\left(\mathcal{Z}_{\varepsilon}^{10 \beta}\right)\right) \cap\left\{u \mid \Upsilon_{\varepsilon}(u) \in \Omega_{\varepsilon}^{8 \beta}\right\}
$$

for some $i=0,1, \ldots, j_{\varepsilon}-1, l \in\left[0, l_{g}\right]$.

In fact, if Case $\mathrm{C}$ does not occur,

$$
\eta_{\varepsilon}\left(l, I^{i}\left(A_{\varepsilon}(t, z)\right)\right) \notin\left(G_{\delta / 2}\left(\mathcal{Z}_{\varepsilon}^{10 \beta}\right) \backslash G_{\delta / 10 \omega}\left(\mathcal{Z}_{\varepsilon}^{10 \beta}\right)\right) \cap\left\{u \mid \Upsilon_{\varepsilon}(u) \in \Omega_{\varepsilon}^{8 \beta}\right\}
$$

for any $i=0,1, \ldots, j_{\varepsilon}-1$ and $l \in\left[0, l_{g}\right]$. Since $\tau_{\varepsilon}\left(G_{c \delta}\left(\mathcal{Z}_{\varepsilon}^{10 \beta}\right)\right) \subset G_{c \delta}\left(\mathcal{Z}_{\varepsilon}^{10 \beta}\right)$ for $c \in(0,2]$ and $P_{\varepsilon}(l, u)=u$ for $l \in\left[0, l_{p}\right]$ and $u \in G_{2 \delta}\left(\mathcal{Z}_{\varepsilon}^{10 \beta}\right) \backslash G_{\delta / 4 \omega}\left(\mathcal{Z}_{\varepsilon}^{10 \beta}\right)$, we see that

$$
\eta_{\varepsilon}\left(l, I^{i}\left(A_{\varepsilon}(t, z)\right)\right) \in G_{\delta / 10 \omega}\left(\mathcal{Z}_{\varepsilon}^{10 \beta}\right) \cap\left\{u \mid \Upsilon_{\varepsilon}(u) \in \Omega_{\varepsilon}^{8 \beta}\right\}
$$

for any $i=0,1, \ldots, j_{\varepsilon}-1$ and $l \in\left[0, l_{g}\right]$. Then, it is obvious from (57) and (58) that Case A or Case B occurs. This proves the claim.

Suppose that Case $\mathrm{C}$ occurs. Then Proposition 15 implies that for small $\varepsilon>0$,

$$
\Gamma_{\varepsilon}\left(I^{j_{\varepsilon}}\left(A_{\varepsilon}(t, z)\right)\right) \leq \Gamma_{\varepsilon}\left(\eta_{\varepsilon}\left(l_{g}, I^{m}\left(A_{\varepsilon}(t, z)\right)\right)\right) \leq E_{m}-v / 2 .
$$

Next we suppose that Case B occurs. Then we see from (56) and Proposition 13 that for small $\varepsilon>0$,

$$
\Gamma_{\varepsilon}\left(P_{\varepsilon}\left(l_{p}, \tau_{\varepsilon}\left(\eta_{\varepsilon}\left(l_{g}, I^{m}\left(A_{\varepsilon}(t, z)\right)\right)\right)\right)\right) \leq E_{m}+o(1)-\mu_{0} \leq E_{m}-\mu_{0} / 2 .
$$

Finally, suppose that Case A occurs. We may assume that

$$
\Gamma_{\varepsilon}\left(\eta_{\varepsilon}\left(l, I^{m}\left(A_{\varepsilon}(t, z)\right)\right)\right) \geq E_{m}-v / 2
$$

for any $m \in\left\{1, \ldots, j_{\varepsilon}-1\right\}$ and any $l \in\left[0, l_{g}\right]$. Note that

$$
\begin{aligned}
\Gamma_{\varepsilon}\left(I^{j_{\varepsilon}}\left(A_{\varepsilon}(t, z)\right)\right)-\Gamma_{\varepsilon}\left(A_{\varepsilon}(t, z)\right) & =\sum_{j=1}^{j_{\varepsilon}}\left[\Gamma_{\varepsilon}\left(I^{j}\left(A_{\varepsilon}(t, z)\right)\right)-\Gamma_{\varepsilon}\left(I^{j-1}\left(A_{\varepsilon}(t, z)\right)\right)\right] \\
\leq & \sum_{j=1}^{j_{\varepsilon}}\left[\Gamma_{\varepsilon}\left(\eta_{\varepsilon}\left(l_{g}, I^{j-1}\left(A_{\varepsilon}(t, z)\right)\right)\right)-\Gamma_{\varepsilon}\left(I^{j-1}\left(A_{\varepsilon}(t, z)\right)\right)\right] .
\end{aligned}
$$


When

$$
\Gamma_{\varepsilon}\left(\eta_{\varepsilon}\left(l, I^{j}\left(A_{\varepsilon}(t, z)\right)\right)\right) \geq E_{m}-v / 2, \quad \Upsilon_{\varepsilon}\left(\eta_{\varepsilon}\left(l, I^{j}\left(A_{\varepsilon}(t, z)\right)\right)\right) \in \Omega_{\varepsilon}^{5 \beta}
$$

for any $j \in\left\{1, \ldots, j_{\varepsilon}-1\right\}$ and any $l \in\left[0, l_{g}\right]$, we have

$$
\frac{d \eta_{\varepsilon}(l, u)}{d l}=-\Gamma_{\varepsilon}^{\prime}\left(\eta_{\varepsilon}\right) /\left\|\Gamma_{\varepsilon}^{\prime}\left(\eta_{\varepsilon}\right)\right\|_{\varepsilon}^{*}
$$

This implies that

$$
\Gamma_{\varepsilon}\left(\eta_{\varepsilon}\left(l_{g}, I^{j-1}\left(A_{\varepsilon}(t, z)\right)\right)\right)-\Gamma_{\varepsilon}\left(I^{j-1}\left(A_{\varepsilon}(t, z)\right)\right) \leq-l_{g} k(\varepsilon) .
$$

Therefore

$$
\Gamma_{\varepsilon}\left(I^{j_{\varepsilon}}\left(A_{\varepsilon}(t, z)\right)\right)-\Gamma_{\varepsilon}\left(A_{\varepsilon}(t, z)\right) \leq-j_{\varepsilon} l_{g} k(\varepsilon) \leq-v ;
$$

thus for small $\varepsilon>0$,

$$
\Gamma_{\varepsilon}\left(I^{j_{\varepsilon}}\left(A_{\varepsilon}(t, z)\right)\right) \leq E_{m}-v / 2
$$

Since

$$
\Gamma_{\varepsilon}\left(I^{j_{\varepsilon}}\left(A_{\varepsilon}(t, z)\right)\right) \leq \Gamma_{\varepsilon}\left(I^{j_{\varepsilon}-1}\left(A_{\varepsilon}(t, z)\right)\right) \leq \cdots \leq \Gamma_{\varepsilon}\left(I\left(A_{\varepsilon}(t, z)\right)\right) \leq \Gamma_{\varepsilon}\left(A_{\varepsilon}(t, z)\right),
$$

it follows that if $\Upsilon_{\varepsilon}\left(\eta_{\varepsilon}\left(l, I^{m}\left(A_{\varepsilon}(t, z)\right)\right)\right) \in \Omega_{\varepsilon}^{5 \beta}$ for any $m \in\left\{1, \ldots, j_{\varepsilon}-1\right\}$ and any $l \in\left[0, l_{g}\right]$, then

$$
\Gamma_{\varepsilon}\left(I^{j_{\varepsilon}}\left(A_{\varepsilon}(t, z)\right)\right) \leq E_{m}-v / 2 .
$$

As a result, we conclude that for small $\varepsilon>0$,

$$
\Gamma_{\varepsilon}\left(I^{j_{\varepsilon}}\left(A_{\varepsilon}(t, z)\right)\right) \leq E_{m}-\min \left\{v / 2, \mu_{0} / 2\right\} \quad \text { for any } t \in[0, T], z \in L_{\varepsilon} .
$$

We define

$$
B_{\varepsilon}(t, z) \equiv \tau_{\varepsilon}\left(I^{j_{\varepsilon}}\left(A_{\varepsilon}(t, z)\right)\right) .
$$

From (44) and the invariance of $\partial G_{2 \delta}\left(\mathcal{Z}_{\varepsilon}^{10 \beta}\right)$ under the flows $\eta_{\varepsilon}$ and $P_{\varepsilon}$ we see that $B_{\varepsilon}$ : $[0, T] \times L_{\varepsilon} \rightarrow H_{\varepsilon}$ is continuous. Proposition 16 implies that if $\varepsilon>0$ is small, for any $t \in[0, T]$ and $z \in L_{\varepsilon}$,

$$
\Gamma_{\varepsilon}\left(B_{\varepsilon}(t, z)\right) \leq \Gamma_{\varepsilon}\left(A_{\varepsilon}(t, z)\right) \leq E_{m}-\min \left\{v / 2, \mu_{0} / 2\right\} .
$$

We note that $B_{\varepsilon}(t, z)=A_{\varepsilon}(t, z)$ for $B_{\varepsilon}(t, z) \notin G_{2 \delta}\left(\mathcal{Z}_{\varepsilon}^{10 \beta}\right)$. Moreover, we see from Propositions 3 and 9 that for small $\varepsilon>0$,

$$
\begin{aligned}
\Gamma_{\varepsilon}\left(B_{\varepsilon}(t, z)\right)= & \frac{1}{2} \int_{\mathbb{R}^{N}}\left[\left|\nabla B_{\varepsilon}(t, z)\right|^{2}+V\left(\varepsilon \tilde{\Upsilon}_{\varepsilon}\left(B_{\varepsilon}(t, z)\right)\right)\left(B_{\varepsilon}(t, z)\right)^{2}\right] d x \\
& -\int_{\mathbb{R}^{N}} F\left(B_{\varepsilon}(t, z)\right) d x+o(1),
\end{aligned}
$$


where $\lim _{\varepsilon \rightarrow 0} o(1)=0$ uniformly for $(t, z) \in[0, T] \times L_{\varepsilon}$. We can take sufficiently small $\delta, T_{0}>0$ so that $B_{\varepsilon}(t, z) \notin N_{2 \delta}\left(\mathcal{Z}_{\varepsilon}^{10 \beta}\right)$ for $(t, z) \in \partial\left(\left[T_{0}, T\right] \times L_{\varepsilon}\right)$. It follows from (43) that for any $t \in[0, T]$,

$$
\varepsilon \tilde{\Upsilon}_{\varepsilon}\left(B_{\varepsilon}(t, z)\right)=\Theta_{\varepsilon}(z) \quad \text { for } z \text { in a neighborhood } \mathcal{N} \text { of } \partial L_{\varepsilon} .
$$

We define

$$
Q_{\varepsilon}(t, z)(u) \equiv \frac{N-2}{2} \int_{\mathbb{R}^{N}}|\nabla u|^{2} d x+N \int_{\mathbb{R}^{N}}\left[\frac{1}{2} V\left(\varepsilon \tilde{\Upsilon}_{\varepsilon}\left(B_{\varepsilon}(t, z)\right)\right)|u|^{2}-F(u)\right] d x
$$

and

$$
J_{\varepsilon}(t, z)(u) \equiv \frac{1}{2} \int_{\mathbb{R}^{N}}\left[|\nabla u|^{2} d x+V\left(\varepsilon \tilde{\Upsilon}_{\varepsilon}\left(B_{\varepsilon}(t, z)\right)\right)|u|^{2}\right] d x-\int_{\mathbb{R}^{N}} F(u) d x .
$$

For $N \geq 3$, it is shown in [7] and [25] that if there exists $h=h(z, t)>0$ satisfying

$$
V\left(\varepsilon \tilde{\Upsilon}_{\varepsilon}\left(B_{\varepsilon}(t, z)\right)\right) h^{2}-2 F(h)<0,
$$

then there exists a minimizer $W=W(t, z)$ of $J_{\varepsilon}(t, z)$ over the Pohozaev manifold $\{u \in$ $\left.H^{1}\left(\mathbb{R}^{N}\right) \backslash\{0\} \mid Q_{\varepsilon}(t, z)(u)=0\right\}$ which is a least energy solution of

$$
\Delta W-V\left(\varepsilon \tilde{\Upsilon}_{\varepsilon}\left(B_{\varepsilon}(t, z)\right)\right) W+f(W)=0, \quad W>0 \quad \text { on } \mathbb{R}^{N} .
$$

For $N=2$, it is shown in [4] that there exists a minimizer $W=W(t, z)$ of $J_{\varepsilon}(t, z)$ over the Pohozaev manifold, and that for some constant $\lambda=\lambda(t, z)>0$, the scaled function $\widehat{W}(x)=W(\lambda x)$ is a least energy solution of

$$
\Delta \widehat{W}-V\left(\varepsilon \tilde{\Upsilon}_{\varepsilon}\left(B_{\varepsilon}(t, z)\right)\right) \widehat{W}+f(\widehat{W})=0, \quad W>0 \quad \text { on } \mathbb{R}^{N} .
$$

Since $Q_{\varepsilon}(t, z)(W)=0$, we see that

$$
J_{\varepsilon}(t, z)(W)=J_{\varepsilon}(t, z)(\widehat{W}) .
$$

We note that for any $z \in L_{\varepsilon}$,

$$
Q_{\varepsilon}\left(T_{0}, z\right)\left(B_{\varepsilon}\left(T_{0}, z\right)\right)>0, \quad Q_{\varepsilon}(T, z)\left(B_{\varepsilon}(T, z)\right)<0
$$

if $T_{0}>0$ is sufficiently small, and that $\Theta_{\varepsilon}(z)=z$ for $z \in L_{0}$. Thus, defining $D_{\varepsilon}(t, z) \equiv$ $Q_{\varepsilon}(t, z)\left(B_{\varepsilon}(t, z)\right)$, we see from (61) that for $z$ in a neighborhood $\mathcal{N}$ of $L_{0}$ in $L_{\varepsilon}$,

$$
\begin{aligned}
& D_{\varepsilon}(t, z) \equiv Q_{\varepsilon}(t, z)\left(B_{\varepsilon}(t, z)\right) \\
& \quad=\frac{(N-2) t^{N-2}}{2} \int_{\mathbb{R}^{N}}\left|\nabla\left(\phi_{\varepsilon} U\right)\right|^{2} d x+N t^{N} \int_{\mathbb{R}^{N}}\left[V\left(\Theta_{\varepsilon}(z)\right)\left(\phi_{\varepsilon} U\right)^{2}-F\left(\phi_{\varepsilon} U\right)\right] d x .
\end{aligned}
$$

This implies that for $z \in \mathcal{N} \subset L_{\varepsilon}, D_{\varepsilon}(t, z)$ is a strictly decreasing function with respect to $t \in\left[T_{0}, T\right]$ if $\varepsilon>0$ is small. Hence, for any $z \in \mathcal{N}$, there exists a unique $t(z) \in\left(T_{0}, T\right)$ such that $D_{\varepsilon}(t(z), z)=0$. Then, it is easy to see that

$$
\frac{\partial D_{\varepsilon}}{\partial t}(t(z), z) \neq 0, \quad z \in \mathcal{N} .
$$


We note that for $s \in\left[T_{0}, T\right]$ and $z \in L_{0}=\partial L_{\varepsilon}$,

$$
\varepsilon \tilde{\Upsilon}_{\varepsilon}\left(B_{\varepsilon}(t, z)\right)=\Theta_{\varepsilon}(z)=z
$$

We will show in the following section that for small $\varepsilon>0$, there exists $\left(t_{\varepsilon}, z_{\varepsilon}\right) \in$ $(0, T) \times L_{\varepsilon}$ satisfying

$$
D_{\varepsilon}\left(t_{\varepsilon}, z_{\varepsilon}\right)=0 \quad \text { and } \quad V\left(\varepsilon \tilde{\Upsilon}_{\varepsilon}\left(B_{\varepsilon}\left(t_{\varepsilon}, z_{\varepsilon}\right)\right)\right)=m
$$

\subsection{Intersection results}

Proposition 17. Let $H$ be a compact connected $k$-dimensional manifold in $\left(T_{0}, T\right) \times L_{\varepsilon}$ such that

$$
\emptyset \neq \partial H \subset\left(T_{0}, T\right) \times L_{0} .
$$

Assume that for the natural projection map $\pi_{\varepsilon}:\left(T_{0}, T\right) \times L_{\varepsilon} \rightarrow L_{\varepsilon}$, the restricted map $\left.\pi_{\varepsilon}\right|_{\partial H}: \partial H \rightarrow \pi_{\varepsilon}(\partial H) \subset L_{0}$ is a homeomorphism. Then $\pi_{\varepsilon}(H)=L_{\varepsilon}$ and $\pi_{\varepsilon}(\partial H)=L_{0}$.

Proof. For the natural projection map $\pi_{\varepsilon}$, we have $\pi_{\varepsilon}: H \rightarrow L_{\varepsilon}$ with $\pi_{\varepsilon}(\partial H) \subset$ $\partial L_{\varepsilon}=L_{0}$. Then, for any $y \in L_{\varepsilon} \backslash L_{0}$, the mod 2 degree $\operatorname{deg}_{2}\left(\pi_{\varepsilon}, H, y\right)$ is well defined. Since $\pi_{\varepsilon}: \partial H \rightarrow \pi_{\varepsilon}(\partial H)$ is a homeomorphism, we see that $\operatorname{deg}_{2}\left(\pi_{\varepsilon}, H, y\right)=1$ for any $y$ close to $\partial L_{\varepsilon}=L_{0}$. Since $L_{\varepsilon}$ is connected and $\pi_{\varepsilon}(\partial H) \subset L_{0}$, we see that $\operatorname{deg}_{2}\left(\pi_{\varepsilon}, H, y\right)$ is independent of $y \in L_{\varepsilon} \backslash L_{0}$. This implies that $\pi_{\varepsilon}(H)=L_{\varepsilon}$. Since $\pi_{\varepsilon}: \partial H \rightarrow \pi_{\varepsilon}(\partial H)$ is a homeomorphism, it follows that $\pi_{\varepsilon}(\partial H)=\partial L_{\varepsilon}=L_{0}$.

Proposition 18. For small $\varepsilon>0$, there exists $\left(t_{\varepsilon}, z_{\varepsilon}\right) \in\left(T_{0}, T\right) \times L_{\varepsilon}$ satisfying

$$
D_{\varepsilon}\left(t_{\varepsilon}, z_{\varepsilon}\right)=0 \quad \text { and } \quad V\left(\varepsilon \tilde{\Upsilon}_{\varepsilon}\left(B_{\varepsilon}\left(t_{\varepsilon}, z_{\varepsilon}\right)\right)\right)=m \text {. }
$$

Proof. We can approximate $D_{\varepsilon}$ in $C^{0}\left(\left[T_{0}, T\right] \times L_{\varepsilon}\right)$ by $\left\{D_{\varepsilon}^{l}\right\}_{l=1}^{\infty} \subset C^{N+1}\left(\left(T_{0}, T\right) \times L_{\varepsilon}\right)$ so that

$$
D_{\varepsilon}^{l}(t, z)=D_{\varepsilon}(t, z) \quad \text { for }(t, z) \in\left(T_{0}, T\right) \times L_{0} .
$$

Then, by Sard's theorem, we can find $\left\{b_{i}^{l}\right\}_{i=1}^{\infty} \subset \mathbb{R}$ with $\lim _{i \rightarrow \infty} b_{i}^{l}=0$ and $b_{i}^{l}$ is a regular value of $D_{\varepsilon}^{l}$. We can take a sequence $\{l(i)\}_{i=1}^{\infty}$ such that $\lim _{i \rightarrow \infty} l(i)=\infty$ and $\lim _{i \rightarrow \infty} b_{i}^{l(i)}=0$. Then, for each $i=1,2, \ldots,\left(D_{\varepsilon}^{l(i)}\right)^{-1}\left(b_{i}^{l(i)}\right)$ is a union of finitely many $k$-dimensional compact connected submanifolds of $\left(T_{0}, T\right) \times L_{\varepsilon}$.

Let $H^{i}$ be a connected component of $\left(D_{\varepsilon}^{l(i)}\right)^{-1}\left(b_{i}^{l(i)}\right)$ which intersects $\left(T_{0}, T\right) \times L_{0}$. Let $\pi_{\varepsilon}:\left(T_{0}, T\right) \times L_{\varepsilon} \rightarrow L_{\varepsilon}$ be the natural projection map. Then, from (63), we see that $\pi_{\varepsilon}: \partial H^{i} \rightarrow \pi_{\varepsilon}\left(\partial H^{i}\right) \subset L_{0}$ is a homeomorphism. Hence, by Proposition 17, $H^{i} \in \mathcal{L}\left(L_{0}\right)$. If $\mathcal{L}\left(L_{0}\right)$ is the class of orientable manifolds, then the $\left\{H^{i}\right\}_{i}$ are orientable since there is a nonvanishing normal vector field on $H^{i}$ in the orientable manifold $L_{\varepsilon} \times$ $\left[T_{0}, T\right]$. Thus, if $\mathcal{L}\left(L_{0}\right)$ is the class of orientable manifolds, we also have $H^{i} \in \mathcal{L}\left(L_{0}\right)$. Then, from the definition of $m$ in (V2), we see that $\max _{(t, z) \in H^{i}} V\left(\varepsilon \tilde{\Upsilon}_{\varepsilon}\left(B_{\varepsilon}(t, z)\right)\right) \geq m$. Since $B_{\varepsilon}(t, z)=A_{\varepsilon}(t, z)$ in a neighborhood of $\partial\left(\left(T_{0}, T\right) \times L_{\varepsilon}\right)$, there exists some $\rho>0$, independent of small $\varepsilon>0$, such that $V\left(\varepsilon \tilde{\Upsilon}_{\varepsilon}\left(B_{\varepsilon}(t, z)\right)\right)<m-\rho$ for $(t, z) \in \partial H^{i}$, and 
$\left|D_{\varepsilon}^{l(i)}\left(T_{0}, z\right)\right| \geq \rho,\left|D_{\varepsilon}^{l(i)}(T, z)\right| \geq \rho$ for $z \in L_{\varepsilon}$ and $i=1,2, \ldots$ Thus there exists $\left(t^{i}, z^{i}\right) \in\left(T_{0}, T\right) \times\left(L_{\varepsilon} \backslash L_{0}\right)$ such that

$$
V\left(\varepsilon \tilde{\Upsilon}_{\varepsilon}\left(B_{\varepsilon}\left(t^{i}, z^{i}\right)\right)\right)=m \quad \text { and } \quad D_{\varepsilon}^{l(i)}\left(t^{i}, z^{i}\right)=b_{i}^{l(i)} .
$$

Let $\left(t_{\varepsilon}, z_{\varepsilon}\right)$ be a limit point of $\left\{\left(t^{i}, z^{i}\right)\right\}_{i}$. Then $\left(t_{\varepsilon}, z_{\varepsilon}\right)$ is an interior point of $\left[T_{0}, T\right] \times L_{\varepsilon}$ since $V\left(\varepsilon \tilde{\Upsilon}_{\varepsilon}\left(B_{\varepsilon}(t, z)\right)\right)<m$ on $\partial\left[T_{0}, T\right] \times L_{\varepsilon}$. Hence,

$$
D_{\varepsilon}\left(t_{0}, z_{0}\right)=0 \quad \text { and } \quad V\left(\varepsilon \tilde{\Upsilon}_{\varepsilon}\left(B_{\varepsilon}\left(t_{0}, z_{0}\right)\right)\right)=m .
$$

Completion of the proof of Theorem 2. We have shown that for small $\varepsilon>0$, there exists $\left(t_{\varepsilon}, z_{\varepsilon}\right) \in\left(T_{0}, T\right) \times L_{\varepsilon}$ satisfying

$$
Q_{\varepsilon}\left(t_{\varepsilon}, z_{\varepsilon}\right)=0 \quad \text { and } \quad V\left(\varepsilon \tilde{\Upsilon}_{\varepsilon}\left(B_{\varepsilon}\left(t_{\varepsilon}, z_{\varepsilon}\right)\right)\right)=m
$$

Note from [7, Proposition 2.1] and [25] that

$$
\begin{aligned}
& E_{m}= \\
& \inf \left\{\left.L_{m}(u)\left|\frac{N-2}{2} \int_{\mathbb{R}^{N}}\right| \nabla u\right|^{2} d x+N \int_{\mathbb{R}^{N}}\left[m \frac{u^{2}}{2}-F(u)\right] d x=0, u \in H^{1}\left(\mathbb{R}^{N}\right) \backslash\{0\}\right\} .
\end{aligned}
$$

Then we see that

$$
J_{\varepsilon}\left(t_{\varepsilon}, z_{\varepsilon}\right)\left(B_{\varepsilon}\left(t_{\varepsilon}, z_{\varepsilon}\right)\right) \geq E_{V\left(\varepsilon \tilde{\Upsilon}_{\varepsilon}\left(B_{\varepsilon}\left(t_{\varepsilon}, z_{\varepsilon}\right)\right)\right)}=E_{m}
$$

since

$$
Q_{\varepsilon}\left(t_{0}, z_{0}\right)\left(B_{\varepsilon}\left(t_{\varepsilon}, z_{\varepsilon}\right)\right)=0 .
$$

This contradicts (59) and (60).

Thus we conclude that for any $d>0$, there exists $\varepsilon_{0}(d)>0$ such that for $\varepsilon \in$ $\left(0, \varepsilon_{0}(d)\right)$, there exists a solution $u_{\varepsilon} \in G_{2 \delta}\left(\mathcal{Z}_{\varepsilon}^{10 \beta}\right)$ of (13) with $\Gamma_{\varepsilon}\left(u_{\varepsilon}\right) \leq C_{\varepsilon}$ and $\Upsilon_{\varepsilon}\left(u_{\varepsilon}\right) \in \Omega_{\varepsilon}^{10 \beta} \subset\left(\mathcal{M}^{d}\right)_{\varepsilon}$. This implies that for small $\varepsilon>0$, there exists a solution $u_{\varepsilon}$ of (13) such that $\lim _{\varepsilon \rightarrow 0} \operatorname{dist}\left(\varepsilon \Upsilon\left(u_{\varepsilon}\right), \mathcal{M}\right)=0$ and $\lim \sup _{\varepsilon \rightarrow 0} \Gamma_{\varepsilon}\left(u_{\varepsilon}\right) \leq E_{m}$. Let $x_{\varepsilon}$ be a maximum point of $u_{\varepsilon}$. Then we see from Proposition 3 that $\lim _{\varepsilon \rightarrow 0} \operatorname{dist}\left(\varepsilon x_{\varepsilon}, \mathcal{M}\right)=0$. Since $\lim \sup _{\varepsilon \rightarrow 0} \Gamma_{\varepsilon}\left(u_{\varepsilon}\right) \leq E_{m}$, we deduce that $u_{\varepsilon}\left(\cdot+x_{\varepsilon}\right)$ converges, up to a subsequence, to a least energy solution $U \in S$ of (4) with $V\left(x_{0}\right)=m$ as $\varepsilon \rightarrow 0$. Thus, the conclusion of Theorem 2 follows.

Acknowledgments. The authors would like to express their sincere thanks to Paul Rabinowitz, Norman Dancer, Manuel del Pino, Patricio Felmer and Louis Jeanjean for their helpful comments and fruitful discussions during the preparation of this paper. The first author was supported by Priority Research Centers Program (No. 2010-0029638) and Mid-career Researcher Program (No. 20100014135) through the National Research Foundation of Korea (NRF) funded by the Ministry of Education, Science and Technology (MEST). The second author is supported in part by Grant-inAid for Scientific Research (B) (No. 20340037, 25287025) of the Japan Society for the Promotion of Science. 


\section{References}

[1] Ambrosetti, A., Badiale, M., Cingolani, S.: Semiclassical states of nonlinear Schrödinger equations. Arch. Ration. Mech. Anal. 140, 285-300 (1997) Zbl 0896.35042 MR 1486895

[2] Ambrosetti, A., Malchiodi, A., Ni, W.-M.: Singularly perturbed elliptic equations with symmetry: existence of solutions concentrating on spheres I. Comm. Math. Phys. 235, 427-466 (2003) Zbl 1072.35019 MR 1974510

[3] Berestycki, H., Lions, P.-L.: Nonlinear scalar field equations I. Arch. Ration. Mech. Anal. 82, 313-346 (1983) Zbl 0533.35029 MR 0695535

[4] Berestycki, H., Gallouët, T., Kavian, O.: Équations de champs scalaires euclidiens non linéaires dans le plan. C. R. Acad. Sci. Paris Sér. I Math. 297, 307-310 (1983) and Publications du Laboratoire d'Analyse Numérique, Univ. de Paris VI (1984) Zbl 0544.35042 MR 0734575

[5] Byeon, J.: Existence of large positive solutions of some nonlinear elliptic equations on singularly perturbed domains. Comm. Partial Differential Equations 22, 1731-1769 (1997) Zbl 0883.35040 MR 1469588

[6] Byeon, J.: Singularly perturbed nonlinear Neumann problems with a general nonlinearity. J. Differential Equations 244, 2473-2497 (2008) Zbl 1148.35028 MR 2414402

[7] Byeon, J.: Singularly perturbed nonlinear Dirichlet problems with a general nonlinearity. Trans. Amer. Math. Soc. 362, 1981-2001 (2010) Zbl 1188.35082 MR 2574884

[8] Byeon, J., Jeanjean, L.: Standing waves for nonlinear Schrödinger equations with a general nonlinearity. Arch. Ration. Mech. Anal. 185, 185-200 (2007) Zbl 1132.35078 MR 2317788

[9] Byeon, J., Jeanjean, L., Tanaka, K.: Standing waves for nonlinear Schrödinger equations with a general nonlinearity: one and two dimensional cases. Comm. Partial Differential Equations 33, 1113-1136 (2008) Zbl 1155.35344 MR 2424391

[10] Byeon, J., Wang, Z.-Q.: Standing waves with a critical frequency for nonlinear Schrödinger equations II. Calc. Var. Partial Differential Equations 18, 207-219 (2003) Zbl 1073.35199 MR 2010966

[11] Cortázar, C., Elgueta, M., Felmer, P.: Uniqueness of positive solutions of $\Delta u+f(u)=0$ in $\mathbb{R}^{N}, N \geq 3$. Arch. Ration. Mech. Anal. 142, 127-141 (1998) Zbl 0912.35059 MR 1629650

[12] Coti Zelati, V., Rabinowitz, P. H.: Homoclinic type solutions for a semilinear elliptic PDE on $\mathbb{R}^{n}$. Comm. Pure Appl. Math. 45, 1217-1269 (1992) Zbl 0785.35029 MR 1181725

[13] Dancer, E. N.: Peak solutions without non-degeneracy conditions. J. Differential Equations 246, 3077-3088 (2009) Zbl 1197.35121 MR 2507950

[14] del Pino, M., Felmer, P.: Local mountain passes for semilinear elliptic problems in unbounded domains. Calc. Var. Partial Differential Equations 4, 121-137 (1996) Zbl 0844.35032 MR 1379196

[15] del Pino, M., Felmer, P.: Semi-classical states for nonlinear Schrödinger equations. J. Funct. Anal. 149, 245-265 (1997) Zbl 0887.35058 MR 1471107

[16] del Pino, M., Felmer, P.: Multi-peak bound states for nonlinear Schrödinger equations. Ann. Inst. H. Poincaré Anal. Non Linéaire 15, 127-149 (1998) Zbl 0901.35023 MR 1614646

[17] del Pino, M., Felmer, P.: Semi-classical states for nonlinear Schrödinger equations: a variational reduction method. Math. Ann. 324, 1-32 (2002) Zbl 1030.35031 MR 1931757

[18] del Pino, M., Felmer, P., Tanaka, K.: An elementary construction of complex patterns in nonlinear Schrdinger equations. Nonlinearity 15, 1653-1671 (2002) Zbl 1022.34037 MR 1925432

[19] del Pino, M., Kowalczyk, M., Wei, J.: Concentration on curves for nonlinear Schrödinger equations. Comm. Pure Appl. Math. 60, 113-146 (2007) Zbl 1123.35003 MR 2270164 
[20] Floer, A., Weinstein, A.: Nonspreading wave packets for the cubic Schrödinger equations with a bounded potential. J. Funct. Anal. 69, 397-408 (1986) Zbl 0613.35076 MR 0867665

[21] Gidas, B., Ni, W.-M., Nirenberg, L.: Symmetry and related properties via the maximum principle. Comm. Math. Phys. 68, 209-243 (1979) Zbl 0425.35020 MR 0544879

[22] Gilbarg, D., Trudinger, N. S.: Elliptic Partial Differential Equations of Second Order. 2nd ed., Grundlehren Math. Wiss. 224, Springer, Berlin (1983) Zbl 0562.35001 MR 0737190

[23] Gui, C.: Existence of multi-bump solutions for nonlinear Schrödinger equations via variational method. Comm. Partial Differential Equations 21, 787-820 (1996) Zbl 0857.35116 MR 1391524

[24] Hirsch, M.: Differential Topology. Grad. Texts in Math. 33, Springer, New York (1976) Zbl 0356.57001 MR 0448362

[25] Jeanjean, L., Tanaka, K.: A remark on least energy solutions in $\mathbb{R}^{N}$. Proc. Amer. Math. Soc. 131, 2399-2408 (2003) Zbl 1094.35049 MR 1974637

[26] Jeanjean, L., Tanaka, K.: Singularly perturbed elliptic problems with superlinear or asymptotically linear nonlinearities. Calc. Var. Partial Differential Equations 21, 287-318 (2004) Zbl 1060.35012 MR 2094325

[27] Kang, X., Wei, J.: On interacting bumps of semi-classical states of nonlinear Schrödinger equations. Adv. Differential Equations 5, 899-928 (2000) Zbl 1217.35065 MR 1776345

[28] Li, Y. Y.: On a singularly perturbed elliptic equation. Adv. Differential Equations 2, 955-980 (1997) Zbl 1023.35500 MR 1606351

[29] Lions, P.-L.: The concentration-compactness principle in the calculus of variations. The locally compact case, II. Ann. Inst. H. Poincaré Anal. Non Linéaire 1, 223-283 (1984) Zbl 0704.49004 MR 0778974

[30] Majer, P.: Two variational methods on manifolds with boundary. Topology 34, 1-12 (1995) Zbl 0819.58003 MR 1308486

[31] Meystre, P.: Atom Optics. Springer (2001)

[32] Oh, Y.-G.: Existence of semiclassical bound states of nonlinear Schrödinger equations with potentials of the class $(V)_{a}$. Comm. Partial Differential Equations 13, 1499-1519 (1988) Zbl 0702.35228 MR 0970154

[33] Oh, Y.-G.: On positive multi-lump bound states of nonlinear Schrödinger equations under multiple well potential. Comm. Math. Phys. 131, 223-253 (1990) Zbl 0753.35097 MR 1065671

[34] H, P.: Rabinowitz. Minimax Methods in Critical Point Theory with Applications to Differential Equations. CBMS Reg. Conf. Ser. Math. 65, Amer. Math. Soc, Providence (1986) Zbl 0609.58002 MR 0845785

[35] Rabinowitz, P. H.: On a class of nonlinear Schrödinger equations. Z. Angew. Math. Phys. 43, 270-291 (1992) Zbl 0763.35087 MR 1162728

[36] Struwe, M.: Variational Methods. Applications to Nonlinear Partial Differential Equations and Hamiltonian Systems. Springer (1990) Zbl 0746.49010 MR 1078018

[37] Tanaka, K.: Periodic solutions for singular Hamiltonian systems and closed geodesics on noncompact Riemannian manifolds. Ann. Inst. H. Poincaré Anal. Non Linéaire 17, 1-33 (2000) Zbl 0955.37040 MR 1743429

[38] Wang, X.: On concentration of positive bound states of nonlinear Schrödinger equations. Comm. Math. Phys. 153, 229-244 (1993) Zbl 0795.35118 MR 1218300 